\title{
Neonatal Abstinence Syndrome in West Virginia: Trends, risks, and effectiveness of in utero exposure detection
}

\author{
Meagan E. Stabler
}

Follow this and additional works at: https://researchrepository.wvu.edu/etd

\section{Recommended Citation}

Stabler, Meagan E., "Neonatal Abstinence Syndrome in West Virginia: Trends, risks, and effectiveness of in utero exposure detection" (2015). Graduate Theses, Dissertations, and Problem Reports. 6711.

https://researchrepository.wvu.edu/etd/6711

This Dissertation is protected by copyright and/or related rights. It has been brought to you by the The Research Repository @ WVU with permission from the rights-holder(s). You are free to use this Dissertation in any way that is permitted by the copyright and related rights legislation that applies to your use. For other uses you must obtain permission from the rights-holder(s) directly, unless additional rights are indicated by a Creative Commons license in the record and/ or on the work itself. This Dissertation has been accepted for inclusion in WVU Graduate Theses, Dissertations, and Problem Reports collection by an authorized administrator of The Research Repository @ WVU.

For more information, please contact researchrepository@mail.wvu.edu. 
Neonatal Abstinence Syndrome in West Virginia: Trends, risks, and effectiveness of in utero exposure detection

Meagan E. Stabler, BS, CHES

\author{
Dissertation submitted \\ to the School of Public Health \\ at West Virginia University \\ in partial fulfillment of the requirements for the degree of \\ Doctor of Philosophy in \\ Epidemiology
}

\author{
Peter R. Giacobbi Jr., PhD, Chair \\ Ilana R. A. Chertok, PhD, MSN, RN, IBCLC \\ Lesley E. Cottrell, PhD \\ Laura R. Lander, MSW, LICSW \\ D. Leann Long, PhD \\ Panitan Yossuck, MD
}

Department of Epidemiology

Morgantown, West Virginia

2015

Keywords: neonatal abstinence syndrome, antenatal drug abuse, medical chart review, screening diagnostics

Copyright 2015 Meagan E. Stabler, BS, CHES 


\section{ABSTRACT \\ Neonatal Abstinence Syndrome in West Virginia: Trends, risks, and effectiveness of in utero exposure detection}

\section{Meagan Stabler}

This dissertation examined neonatal abstinence syndrome (NAS) in West Virginia (WV). Specifically, three studies were conducted that investigated state and regional trends, risks related to NAS, and effectiveness of in utero exposure detection. The first study described regional incidence rates, trends of antenatal drug class-specific exposures, and NAS diagnoses in WV between 2007 and 2013. The findings indicated a 4-fold increase in WV statewide incidence rates of NAS during this time period, which was three times the national annual average. The second study identified the risk of NAS, risk of NAS requiring pharmacologic treatment, and odds of NAS controlling for potential confounders among substance-using pregnant women who gave birth at Ruby Memorial Hospital between January $1^{\text {st }} 2009$ and March $3^{\text {rd }} 2014$. Results indicated that multiple substances could lead to a NAS diagnosis and that variations existed between drug class-specific exposure and heightened risk of NAS and NAS requiring pharmacological treatment. The third study assessed the effectiveness of maternal urine drug screens, maternal substance use ICD-9-CM diagnostic codes, and neonatal $I C D-9-C M$ exposure codes in detecting antenatal substance use among a subpopulation from the second study. These alternative methods performed poorly, with low sensitivity values, when compared to a meconium analysis (i.e., a neonatal non-invasive biological drug screen). Therefore, solely utilizing alternative screening methods taken at labor and delivery would likely underestimate the number of exposed newborns. This is important because unidentified newborns could be released from the hospital prior to their withdrawal symptom onset and/or left medically untreated. Taken together, these findings provide both research and clinically relevant implications regarding trends of NAS in WV, drug class-specific risk of NAS and NAS requiring pharmacological treatment, and the effectiveness of in utero exposure detection. 


\section{Table of Contents}

Chapter 1: Overview of the research

1.1 Substance use in pregnancy .......................................................................... 1

1.2 Neonatal abstinence syndrome ………………………................................... 3

1.3 Toxicology screening ...................................................................................... 5

1.4 Gaps in the literature.......................................................................................... 7

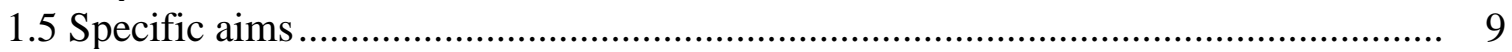

1.6 Overview of methodologic approach................................................................. 10

Chapter 2: Manuscript 1

Neonatal Abstinence Syndrome in West Virginia Sub-State Regions, 2007-2013 .................... 13

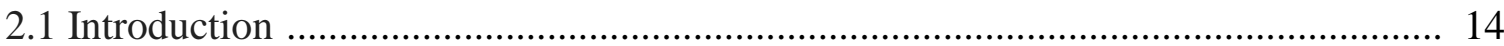

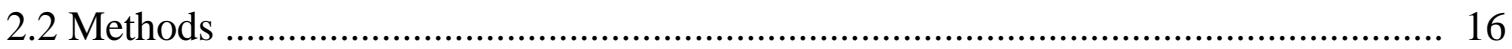

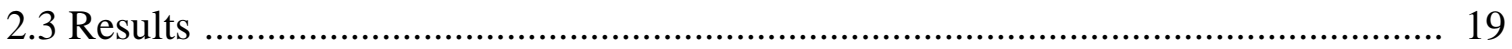

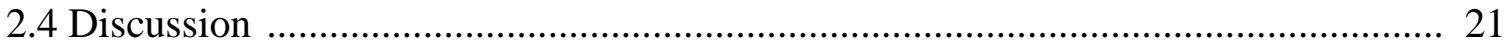

Chapter 3: Manuscript 2

Exposure to substance use in utero, risk of neonatal abstinence syndrome, and antenatal correlates: A retrospective medical chart review ................................................................... 28

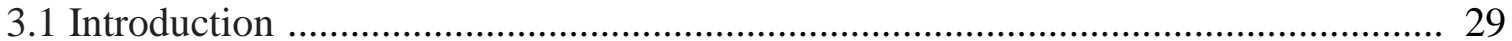

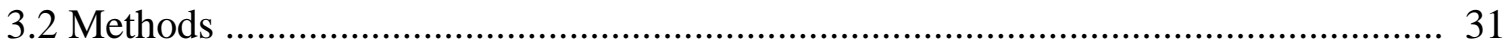

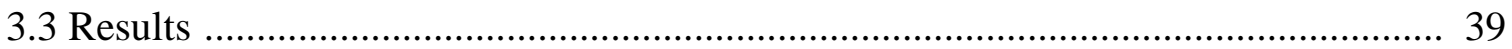

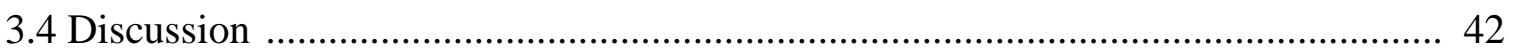

Chapter 4: Manuscript 3

Assessment of in utero opiate and cocaine exposure detection: A comparison of meconium analysis to alternative maternal and neonatal biological and diagnostic indicators .................... 50

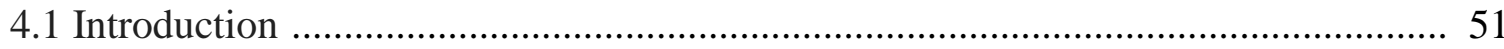

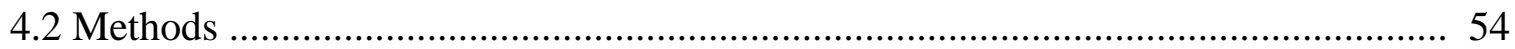

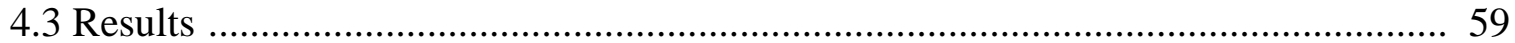

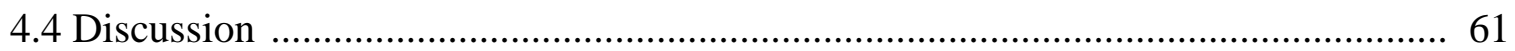

Chapter 5: Discussion

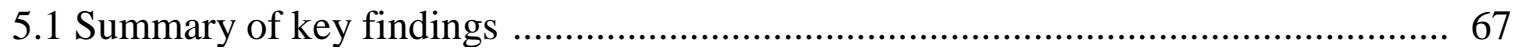

5.2 Significance of the study .............................................................................. 70

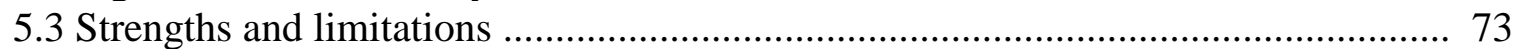

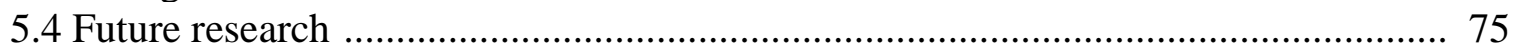

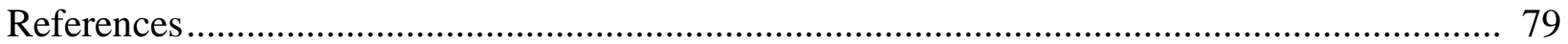

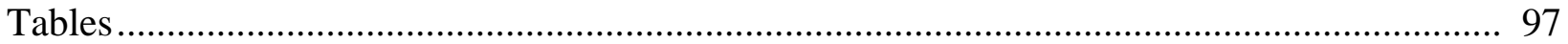

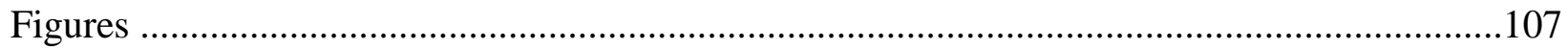

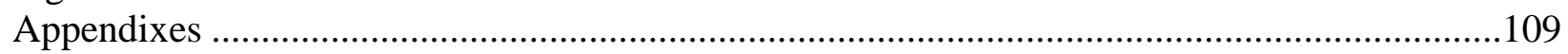

A. $\quad$ Modified Finnegan Scoring System....................................................................111 
B. Neonatal abstinence syndrome treatment guideline and dosage regiment throughout the duration of the study period at Ruby Memorial Hospital in

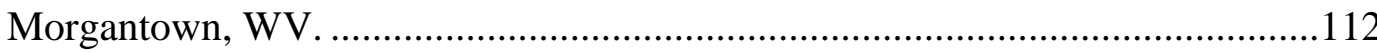

C. Research Electronic Data Capture (REDCAP) data collection tool ...................118

D. REDCAP data collection tool for meconium and umbilical cord tissue .............119

E. Meconium and umbilical cord tissue drug cutoff concentrations, ARUP

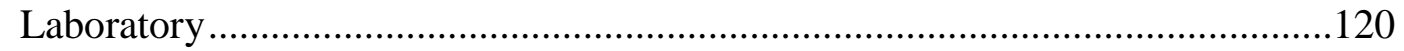

F. Chapter 4 appendix table: Diagnostic equations and cell counts of in utero drug exposure by the four screening methods. 


\section{List of Tables}

$\underline{\text { Table }}$

$\underline{\text { Page }}$

2.1. West Virginia delivery characteristics by neonatal abstinence syndrome: HCA,UB 20072013.

3.1 Breakdown of neonatal biological drug screen results and drug-class exposure groups...... 98

3.2 Risk of neonatal abstinence syndrome (NAS) and pharmacological treatment of NAS among a cohort of substance-using mother-infant dyads.

3.3 Maternal and neonatal characteristics by neonatal abstinence syndrome diagnosis........ 100

3.4 Association between in utero substance exposure and neonatal abstinence syndrome.

$(n=279)$

4.1. Meconium sample required and drug cutoff concentrations

4.2. Maternal and neonatal characteristics by meconium results $(n=381)$

4.3. Estimates of in utero drug exposure in 381 mother-infant dyads by neonatal meconium, maternal UDS, and diagnostic codes.

4.4. Detection of in utero opioid and cocaine exposure among maternal urine drug screen, substance use diagnosis, and neonatal exposure diagnosis in comparison to meconium drug test. 


\section{List of Figures}

Figure $\quad$ Page

Figure 2.1. SAMSHA West Virginia region and opioid treatment program center map ...........107

Figure 2.2. West Virginia regional and hospital neonatal abstinence syndrome incidence rate per

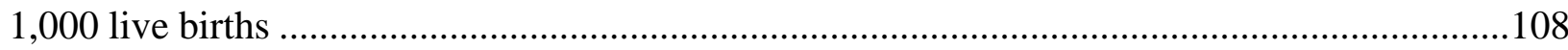

Figure 2.3: Number of inpatient hospitalizations resulting from exposure to noxious substances,

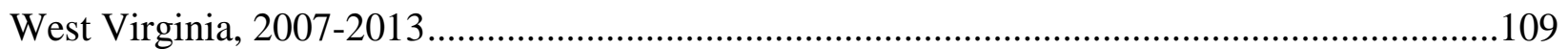

Figure 3.1. Biopsychosocial approach to understanding neonatal abstinence syndrome ............110 


\section{Chapter 1}

\section{Overview of the Research}

\subsection{Substance Use in Pregnancy}

Substance use during pregnancy is a growing phenomenon associated with negative health outcomes for mothers, as well as newborns. In 2012-2013 the national annual average of self-reported illicit drug, alcohol, and tobacco use among pregnant women aged 15 to 44 years was $5.4 \%, 9.4 \%$, and $15.4 \%$, respectively.[1] Rates of illicit drugs, alcohol, and tobacco use decreased with older maternal age and were lower among pregnant women in their third trimester compared to women in their first and second trimesters.

Although national rates decreased with maternal age and trimester during pregnancy, the rate of reported illicit drug use among pregnant women aged 15 to 44 years increased from $4.4 \%$ in 2010 to $5.4 \%$ in the 2012-2013 annual average.[2] The rise in national trends of antenatal illicit drug use is also illuminated by the increased proportion of pregnant women admitted for substance abuse treatment for drug abuse only (i.e., not alcohol). Specifically, treatment admissions for drug abuse increased from $51.1 \%$ in 2000 to $63.8 \%$ in 2010.[3]

In the state of West Virginia (WV), drug screening estimates have revealed that $19.2 \%$, or one in five, newborns are exposed to licit and/or illicit drugs in utero.[4] Although surprising, this statistic is in line with the state's drug epidemic. In 2012, WV had the highest age-adjusted death rate for drug poisonings and the third highest prescribing rate of opioid analgesics in the country.[5] The state's high neonatal exposure, drug overdose, and prescribing rates indicate that the drug epidemic has influenced one of the most vulnerable populations, pregnant women and their unborn children. 
Nearly all noxious substances used during pregnancy can induce drug-related adverse events. Largely, obstetric and neonatal health outcomes of in utero substance exposure include miscarriage, developmental defects, premature birth, and behavioral and cognitive complications.[6] Drugs consumed by the mother can readily transfer across the placenta to the fetus. The extent of placenta transfer varies base on the substances molecular weight, the $\mathrm{pH}$ when the drug is $50 \%$ ionized, and the drug's ability to bind to the plasma protein.[7] The influence of dosage effects is controversial, as it is not always true that higher doses of a noxious substance is more deleterious than lower doses. Dosage effects on the embryo/fetus depend on when exposure occurred during the developmental stage, the dose level and pattern of sequential administration, as well as the route of administration.[8] Other variables that influence defects in the fetus after in utero substance exposure include the stage of embryonic development, genotype of the mother and fetus, drug interactions, and other obstetric factors.[8]

There are three stages of susceptibility associated with embryonic development. In the first stage, or embryogenesis, the drug can be lethal, but surviving embryos are not likely to develop associated malformations (first two weeks of pregnancy).[9] The second stage of organogenesis or organ differentiation is when the embryo is most susceptible to fetal malformations ( 3 to 8 weeks of pregnancy).[8] In addition, the spectrum of anomalies can be determined from the specific timing of exposure, as teratogens affect organ systems at organspecific stage of development. The third stage of embryonic development, called organ growth, is where noxious influences can impact fetal growth but does not directly alter organogenesis ( 8 to 10 weeks of pregnancy).[8] After embryogenesis, the brain, gonadal tissues, and lungs continue to develop in the second and third trimester and therefore in utero exposure to noxious 
substances at that time can negatively impact the fetus.[10] Use of substances throughout the entire pregnancy can also result in neonatal withdrawal.[11]

\subsection{Neonatal Abstinence Syndrome}

An increase in antepartum drug use is also made evident by vast increase in neonatal abstinence syndrome (NAS). NAS is a constellation of central nervous system, metabolic vasomotor, respiratory, and gastrointestinal disturbances a neonate experiences resulting from drug withdrawal following delivery $[11,12]$. NAS most commonly results from in utero substance exposure, but postnatal NAS from discontinuation of analgesic medications in the newborn, or iatrogenic NAS, can also result in a diagnosis.[13, 14] Associated symptoms of NAS include high-pitched crying, jitteriness, convulsions, tremors, sweating, fever, mottling, excessive sucking or rooting and therefore poor feedings, vomiting, and diarrhea. Severe cases can result in seizures and, although rare, withdrawal can indirectly result in neonatal death.[15]

Despite the associated health ramifications of NAS, the incidence has greatly increased, which has strained fiscal and hospital management resources. Between 2000 and 2012 the national incidence of NAS increased nearly 500\% from 1.2 to 5.8 per 1000 hospital births per year.[16, 17] This sharp increase is most likely due to the prescription opioid epidemic.[17, 18] Opioid pain reliever prescriptions grew 4-fold in the last decade, which was paralleled by an increase in opioid-related overdose deaths.[19] The prescription opioid epidemic has infiltrated America's pregnant population, as 1 in 5 pregnant women filled an opioid prescription between 2000 and 2007.[20, 21]

In addition, estimates adjusted for inflation, approximate that the average medical cost of delivering a NAS baby increased from $\$ 39,400$ in 2000 to $\$ 53,400$ in 2009 , of which $77.6 \%$ of costs were charged to respective state Medicaid programs.[17] The total US public health and 
medical cost resulting from babies born with drug withdrawal in 2009 was between 70.6 and 112.6 million dollars.[18] In addition, neonatal intensive care units are often ill-equipped to handle the influx of neonates born with drug withdrawal. An average of one baby is born per hour with drug withdrawal in the United States and the mean length of hospital stay for these newborns is 16 days, compared to non-NAS diagnosed infant of 3 days.[17]

Neonatal withdrawal most commonly results from in utero opioid exposure, although other drug exposures have been linked to NAS diagnoses.[17, 22] Alcohol [23-25], nicotine [2629], cocaine [30-32], phencyclidine (or PCP) [33], methamphetamine [34], benzodiazepine [35, 36], and other psychotropic medication [37-40] antenatal exposures produce similar withdrawallike signs and symptoms and thus neonates with these exposures have been diagnosed with NAS. Among neonates with antenatal opioid exposure, 55\% to $94 \%$ develop withdrawal symptoms and are then closely observed for diagnosis and/or treatment.[11]

In 2014, WV (the state in which the studies were conducted) held a statewide meeting where neonatologists, insurance, hospital, perinatal partnership, and WV Department of Health and Human Resource officials reached consensus on a standardized definition and diagnostic criteria for NAS. Representatives agreed that NAS includes withdrawal from opioid and nonopioid substances and the definition is not limited to cases that require pharmacological treatment. Rather, cases are defined on a known in utero exposure and clinical signs and symptoms of withdrawal. Neonates considered high-risk for NAS are those who exhibit withdrawal symptoms, have a positive neonatal drug screen, or are born to mothers identified with a substance use disorder, who exhibit signs of drug use, lack prenatal records/visits, or have a positive urine drug screen. Additional clinical conditions that indicate high-risk neonates include intrauterine growth retardation, preterm delivery, abruptio placentae, or cardiovascular 
accidents in the mother or infant. High-risk neonates require stringent pediatric observation, toxicology testing, and immediate NAS scoring to determine severity and treatment.

At the study hospital, NAS severity and treatment protocols were assessed via the Modified Finnegan Scoring system (see Appendix A).[41] All high-risk neonates were evaluated prior to feeding, every 2 to 4 hours after birth. The total Finnegan score (46 max) is the sum of 21 individual scores calculated by presence and severity of symptoms associated with in utero opioid exposure. NAS was diagnosed when an infant scores $\geq 8$ during two consecutive screenings. Non-pharmacologic treatment occurred at the onset of diagnosis, which included kangaroo care, swaddling, high caloric diet, and a low-stimuli environment. Pharmacological treatment was necessary when NAS scores were more severe (i.e., infants with a score of $\geq 12$ or greater on three consecutive occasions or a combined score of $\geq 28$ ). The standard treatment was oral morphine solution with an optional adjunctive treatment of phenobarbital. Dosage regiments throughout the process of initiation to cessation of treatment can be referred to in appendix B. The primary goal was to cease NAS treatment and reach infant rhythmic feeding, sleeping, and weight gain with the least amount of medication.

\subsection{Toxicology Screening}

Detection of maternal substance use during pregnancy is necessary, in order to initiate substance abuse treatment and allocate education and support resources for the soon to be mother. For example, methadone or buprenorphine opioid maintenance therapy (OMT) is the standard of care for women with opioid use disorders during pregnancy.[12] Although OMT often results in NAS, overriding benefits for the mother-infant dyad include stabilization on the drug to avoid illicit opioid use and fetal in utero withdrawal during pregnancy, encouraged prenatal care and participation in other educational interventions, and reduced associated 
criminal and risk-based activities.[12] In addition, NAS is an expected and treatable condition among neonates born to women enrolled in OMT.

Identification of antenatal substance use is also beneficial to health care providers because it enables them to better prepare for potential obstetric and neonatal clinical implications at birth, and at-risk neonates would be identified for immediate NAS observation, treatment, and extend hospital length of stay.[12] Extending neonatal hospital length of stay is important because, depending on various drug exposures, environmental and genetic factors, withdrawal often occurs 48 to 72 hours after delivery. A neonate could be mistaken as healthy and discharged home prior to the start of withdrawal symptoms.[42]

Toxicology testing is indicated in high-risk mothers and neonates, as described in section 1.2. Maternal urine drug screens (UDS) during pregnancy is a traditional method of toxicology testing. UDS is encouraged to take place at the first and sequential prenatal care visits throughout pregnancy. The drug screen can still miss women who are positive for antenatal substance use with this level of screening [43, 44], therefore in 2011 the study hospital adopted a policy of universal maternal UDS screen at labor and delivery hospital admission. This type of screening can feasibly be conducted in mass quantities at delivering hospitals with a quick turnaround time to provide clinician indications of antenatal substance use.[45] Limitations of UDS include the short window of detection (i.e., metabolites may detect a few days to a few weeks with maternal UDS, neonatal UDS can only detect maternal antenatal use a few day prior to delivery) and there are challenges with collecting neonatal urine because the collection bag regularly irritates the infants' skin, often failing to adhere.[45]

Meconium is the specimen of choice for detecting neonatal in utero exposure. It is advantageous because of the associated long window of detection (i.e., $12^{\text {th }}$ to $16^{\text {th }}$ week of 
gestation onward).[45] Meconium testing is also feasible with costs per unit similar to that of neonatal UDS.[46, 47] Unfortunately, receipt of meconium test results can take up to three days due to specimen delay, confirmation of positives are necessary due to the potential for falsepositive results, and specimen samples can be compromised and/or unavailable.[48]

Umbilical cord tissue (UCT) drug screens are comparable to meconium in that they have the same long window of detection and they have high agreement (greater than 90\%) on amphetamines, opioids, cocaine, cannabinoids, and phencyclidine in utero exposure detection.[49, 50] An additional benefit of UCT drug screens are their fast turnaround testing time and the ability to collect an uncompromised specimen sample on every neonate.[48] UCT is considered a new alternative method of measuring in utero exposure, thus less is known about disposition of drugs in the specimen, which makes it more difficult to interpret results.[45] In March 2013, the study hospital enrolled in a quality improve protect and changed their neonatal biological drug screening protocol from meconium specimens of at-risk neonates to universal UCT screening. This change was largely due to the perceived benefit of universal screening[4] and the ability of UCT to specifically detect buprenorphine, which was previously undetectable in meconium.

\subsection{Gaps in the Literature}

Due to the rise in opioid (prescription and illicit) drug misuse and NAS, this area of research has gained national attention. There is evidence-based treatment for pregnant women with opioid use disorders, which includes methods for maternal screening, OMT initiation, and maternal cross-disciplinary care.[12, 51, 52] Standards of care also exist for evaluation, assessment, and treatment of infants diagnosed with NAS, though guidelines are tailored to an opioid in utero exposure.[53, 54] 
Generally, NAS is defined in the context of mothers with opioid use disorders and/or mothers who are treated via buprenorphine or methadone OMT during pregnancy. Therefore, NAS among neonates with non-opioid in utero exposures are not typically captured in the literature.[55] To date, few studies have compared NAS severity across maternal drug using (i.e., groups of opioid and opioids with other substances).[56-61] Only one other study assessed risk of NAS requiring treatment among opioid and non-opioid exposed groups, though the study population consisted solely of neonates who were diagnosed with NAS.[62] Assessment of maternal poly-substance use with opiates or by multiple drug groups and neonatal outcomes, other than NAS, have also been measured.[63-67]

NAS rates are typically reported in the literature via hospital, state, or national settings. West Virginia state and sub-state NAS rates and trends of NAS are not currently documented. If the distribution of NAS diagnoses were indicated by patient's residence, then prevention efforts and health care services could more appropriately allocate resource to populations that could benefit most.

To the authors' knowledge, four studies have compared the effectiveness of in utero substance exposure detection among alternative screening methods.[42, 46, 68, 69] Of these studies, three assessed meconium drug screening and two utilized meconium and screened for cocaine and opiates exposure. The current study is the first to compare the effectiveness of alternative screening methods at labor and delivery admission and to determine diagnostic effectiveness of maternal substance use (International Classification of Diseases, 9th Revision, Clinical Modification) ICD-9-CM codes and neonatal ICD-9-CM exposure codes compared to meconium results. 


\subsection{Specific Aims}

The overarching goal of this dissertation and future research is to better understand the risk and trend of NAS, as well as the effectiveness of in utero substance exposure detection methods. Knowledge generated will help guide public health interventions, the allocation of resources, and accurate identification of exposed neonates to ensure proper observation and treatment. Specifically, this dissertation is composed of three separate but related studies that aims to:

- $\quad$ Chapter 2: Describe regional incidence rates and trends of neonatal drug class-specific in utero exposure and NAS diagnoses WV between 2007 and 2013.

- $\quad$ Chapter 3: Identify the risk of NAS, risk of NAS requiring pharmacological treatment, and odds of NAS controlling for potential confounders among substance-using pregnant women who gave birth at Ruby Memorial Hospital between January 1, 2009 and March $3,2014$.

- Chapter 4: Assess the effectiveness of maternal urine drug screens, maternal substance use $I C D-9-C M$ diagnostic codes, and neonatal $I C D-9-C M$ exposure codes in detecting antenatal substance use among substance-using pregnant women who gave birth at Ruby Memorial Hospital between January 1, 2009 and March 3, 2014.

Chapter 3 utilized the biopsychosocial model as a theoretical framework, in which to structure the selection of potential risk factors for NAS (see Figure 3.1). The biopsychosocial model assesses health-related outcomes via a holistic multi-faceted approach. Biologic, psychological, and socioeconomic factors of the mother-infant dyad associated with NAS and specific maternal substance use were identified.[70] In order to calculate the least-biased 
estimate of the effect of in utero drug class-specific exposure on the odds of NAS, confounding was explored via the identified factors and controlled in the analysis.

\subsection{Overview of Methodologic Approach}

The first study (chapter 2) utilized the WV Health Care Authority, Uniform Billing Database for years 2007 to 2013. This data was linked by region of patient residence, in order to calculate regional rates and trends. Data collection for this study started in 2007 because single diagnostic reports from the HCA prior to that date were unreliable. The HCA data exclusion criteria were HIV discharges, admission type not equal to 4 (newborn), residency in state other than $\mathrm{WV}$, and any diagnostic code equal to ICD-9-CM code 779.9 (stillborn). HIPAA compliance measures did not allow the HCA to disseminate information on newborns diagnosed with HIV. Multiple birth pregnancies are counted as separate live births. The outcome was defined as a primary or secondary NAS diagnosis, via $I C D-9-C M$ code 779.5 , labeled drug withdrawal syndrome in newborn. HCA de-identified data was available upon request, once a limited data agreement that ensured HIPAA privacy standards were met. Data could only be released if the minimum cell size was 30 or greater. Due to the relatively small occurrence of NAS cases among the 55 counties in WV each year, the data was aggregated by regions. The six geographic sub-state regions were defined from the Substance Abuse and Mental Health Services Administration (SAMHSA) and reported in 2008-2010 National Survey of Drug Use and Health (NSDUH) report. This report found different variations of mental health and substance use problems among the six sub-state regions in WV.[71]

The second and third studies conducted a retrospective medical chart review of substance-using pregnant women who gave birth between January $1^{\text {st }} 2009$ and March $3^{\text {rd }} 2014$ at Ruby Memorial Hospital in Morgantown, WV. Electronic medical records via the hospital's 
EPIC (i.e., WVU's electronic medical record system) were reviewed to determine previous maternal drug exposures and subsequent maternal and neonatal outcomes. A WV University Hospital decision support analyst generated a mother-infant dyad study sample in accordance to the studies eligibility criteria, which contained patients' medical record number (MRN) and other study variables that were readily available. Four additional codes then manually extracted additional retrospective data via the patients MRN identifier in EPIC and entered/stored the data in REDCap (Research Electronic Data Capture), which is a HIPAA compliant and protected data collection tool (see appendix C and D). In order to maintain confidentiality of data and privacy of subjects, arbitrary study IDs were randomly assigned to each mother-infant dyad and one master list was created that linked original participant MRNs to study IDs. This master list is permanently kept in a secure and protected file within REDCap.

Descriptive summaries and prevalence estimates were assessed using frequencies, proportions, means, and standard deviations, as well as non-parametric equivalents for nonnormal data. Differences in proportions of NAS diagnosis and NAS requiring treatment among maternal and neonatal characteristics were examined using the Pearson Chi-square and Student's t-test. When appropriate, the non-parametric and exact equivalent tests were conducted.

The first study visually demonstrated NAS incidence rates via annual comparative WV region maps utilizing ArcGIS 10.2 software. In addition, the Mantel-Haenszel Chi-square $\left(\chi^{2} \mathrm{MH}\right)$ test was used to test for associated when NAS incidence rates were stratified by both region and hospital and the Cochran-Armitage test (Z) for trend was utilized to test for linear trends in the proportion of NAS diagnosis from 2007 to 2013.[72]

In the second study potential confounders were identified via the change-in-estimate method; if the effect estimate change was $10 \%$ or more, the covariate was included in the logistic 
regression model.[73] The third study compared the performance of alternative drug screening methods to meconium test results via sensitivity, specificity, positive predictive value, negative predictive value, and accuracy measures. Binomial proportion estimates and asymptotic standard error (ASE) 95\% confidence intervals were reported. Data management and analyses were conducted using SAS 9.4 (SAS Institute Inc., Cary, North Carolina) and significance was defined with a 2-tailed alpha of .05. 


\section{Chapter 2}

Neonatal Abstinence Syndrome in West Virginia Sub-State Regions, 2007-2013

\section{Abstract}

Objective: The opioid epidemic is a public health threat with consequences affecting newborns. Neonatal Abstinence Syndrome (NAS) is a constellation of withdrawal symptoms resulting primarily from in utero opioid exposure. Nationwide estimates show a sharp increase in NAS; however, minimal research has assessed NAS and neonatal exposure in WV. Method: The 20072013 WV Health Care Authority, Uniform Billing Data were analyzed for 119,605 newborn admissions with 1,974 NAS diagnoses. NAS (ICD-9-CM 779.5), expressed as incidence rate (IR) per 1,000 live births, and exposure diagnostic codes (opioids (760.72), hallucinogens (760.73), and cocaine (760.75)) were utilized. Results: Between 2007 and 2013, NAS IR significantly increased from 7.74 to 31.56 per 1,000 live births per year $(\mathrm{Z}:-19.10, \mathrm{P}<.0001)$. During this time period, opioid exposure increased (Z: $-9.56, \mathrm{P}<.0001)$, while cocaine exposure decreased (Z: $3.62, P=.0003)$. In 2013 , the southeastern region of the state had the highest NAS IR of 48.76 per 1,000 live births. NAS infants were more likely to experience other clinical conditions, longer hospital length of stay, and be insured by Medicaid. Conclusion: Statewide NAS IR and exposure has greatly increased since 2007. This alarming trend is deleterious for the health of WV mother-child dyads and it strains the state's healthcare system. Therefore, WV has a unique need for prenatal public health drug treatment and prevention resources, specifically targeting the rural southeastern region. Further examination of maternal drug-specific use trends and general underutilization of neonatal exposure $I C D-9-C M$ codes is indicated. 


\subsection{Introduction}

With the increase in opioid prescribing and prescription overdose deaths, it is evident that an opioid epidemic has emerged in the United States (US).[74] This epidemic has far reaching consequences that has affected one of the most vulnerable populations, pregnant women and infants. Neonatal abstinence syndrome (NAS) is a constellation of signs and symptoms of withdrawal that occur in newborns, as a result of illegal or prescription in utero drug exposure.[12] NAS-like symptoms are characterized as central nervous system, gastrointestinal, respiratory, and autonomic disturbances.[11, 12] Specific signs and symptoms include: irritability, feeding difficulties, excessive sucking and/or crying, hyperactive reflexes, sleep problems, vomiting, diarrhea, and sometimes seizures. NAS most commonly results from antepartum opiate use, but the diagnosis has occurred in the context of other illicit and prescription drugs.[22] The syndrome is diagnosed in 55\% to $94 \%$ of newborns exposed to opioids in utero and it is commonly a comorbid diagnosis with other conditions such as low birthweight, preterm birth, and intrauterine growth retardation.[11] In addition, the average medical cost of delivering a NAS infant reached $\$ 1.5$ billion in 2012 , with $81 \%$ of costs charged to respective state Medicaid programs.[16]

From 2000 to 2009 and 2000 to 2012, maternal opiate use during pregnancy increased from 1.19 to 5.63 per 1,000 hospital births, and the incidence of NAS in the US increased nearly $500 \%$ from 1.2 to 5.8 per 1,000 hospital births per year, respectively.[16, 17] In 2012, nationwide geographic variations in NAS showed that the largest rates occurred in the East South Central parts of the country (Kentucky, Tennessee, Mississippi, and Alabama) with 16.2 per 1,000 live births.[16] Minimal research has addressed NAS and neonatal in utero substance exposure in rural settings, as granularity of the geographic variation in NAS study findings do not include WV specific rural state or sub-state rates. 
Although substance use in pregnancy is a concern nationwide, the state of WV faces distinct challenges due to issues related to rurality, poverty [75], drug use, and other factors influencing poor infant health outcomes. WV is ranked as the third most rural state, with a population of 950,184 residing in 2010 Census Bureau defined rural areas.[76] In addition, it is the only state completely immersed within the Appalachian region.[77] The top 5 ranked states for age-adjusted drug overdose mortality rates (i.e., WV, Kentucky, New Mexico, Nevada, and Utah) are designated as predominately rural states by the Office of Rural Health Policy.[78, 79] Specifically, WV has the highest age-adjusted death rate from drug poisoning in the country (36.3 per 100,000 population) and the third highest prescribing rate of opioid analgesics (137.6 per 100 people).[5] Substance use during pregnancy is more common among women residing in rural areas compared to urban areas.[80-83] Rural pregnant women have increased rates of prescription opiate, benzodiazepine, and injection drug use compared to pregnant women from urban areas.[81] In addition to increased drug use, pregnant women with opioid use disorder are faced with societal stigmas and lowered accessibility to substance abuse treatment in rural areas where lack of resources and greater disparities exist.[84-87]

Infants born to women residing in rural areas are at an increased risk of presenting adverse birth outcomes.[84] Compared to the rest of the US, WV statistics provide additional evidence emblematic of poor infant health outcomes: higher teen (15-19 years of age) birth rates (4.5\%), higher prenatal smoking rates $(26.3 \%)$, and higher cesarean section delivery rates (36.5\%).[88] In 2014, the state was ranked $11^{\text {th }}$ nationally for infant mortality, $7^{\text {th }}$ for percent of low birthweight babies, and $13^{\text {th }}$ for percent of preterm live births.[75] In terms of neonatal substance exposure, a 2009 statewide study of cord tissue samples showed that $19.2 \%$ of infants were antenatally exposed to licit/illicit drugs and alcohol, excluding nicotine.[4] 
With the increase in antepartum opiate use, NAS diagnosis, and hospital utilization, NAS is a major public health concern. There is no WV statewide or regional estimate of the incidence of NAS, thus the purpose of the current study is to outline the scope of the problem in the primarily rural state of WV. The primary objectives were to examine state- and region-level NAS and drug-specific trends between 2007 and 2013, as well as to present infant patient characteristics associated with NAS and hospital-level NAS rates for WV birthing hospitals.

\subsection{Methods}

\section{Data Source and Identification of Sample}

A serial cross-sectional analysis was conducted with data from the WV Health Care Authority (HCA), Uniform Billing Database (UB). The claims data were collected at the conclusion of every year from 2007 to 2013. The HCA provided de-identified hospital inpatient discharges of WV resident newborn admissions that were delivered in-state, excluding stillborn (ICD-9-CM code 779.9) and HIV diagnoses. Multiple birth pregnancies were counted as separate live births. The UB data reflects the final amount billed/charged on the newborn's claim and not the total cost of delivery. Therefore, an associated healthcare expenditure analysis was not conducted.

Infants born in hospitals that delivered 10 newborns or less within the 7 year period were excluded from the study $(n=15)$. This helped eliminate bias, as these infants could have been diagnosed and treated differently than those delivered at registered birthing centers. In addition, newborns were excluded from the study $(n=758)$ if they were diagnosed with any of the following conditions: intraventricular hemorrhage (ICD-9-CM, 772.1x), periventricular leukomalacia (ICD-9-CM, 779.7), necrotizing enterocolitis (ICD-9-CM, 777.5x), spontaneous intestinal perforation (ICD-9-CM, 777.6), or bronchopulmonary dysplasia (ICD-9-CM, 770.7). 
These newborns were likely to have prolonged length of stay in the hospital and were more likely to have received opiate medication resulting in iatrogenic NAS. Likely iatrogenic NAS cases were excluded from the study because the etiology of withdrawal differs from antenatal exposed NAS.[17] Total excluded cases equal less than $1 \%$ of the baseline population (i.e., $0.99 \%$ of 120,378 observations).

\section{Outcome}

NAS incidence rate (IR) per 1,000 live births was the outcome of interest. NAS (yes, no) was defined via the presence of an ICD-9-CM 779.5: 'Drug withdrawal syndrome in newborn' diagnosis (diagnostic fields 2-18). In order to comply with the federal Health Insurance Portability and Accountability Act (HIPPA) privacy standards, the data were aggregated by 6 geographic sub-state regions pre-defined by the Substance Abuse and Mental Health Services Administration (SAMHSA) 2008-2010 NSDUH report (see Figure 2.1). The SAMHSA report outlined unequal distributions of mental health and substance use occurrence among the sub-state regions.[71]

Neonatal substance exposure diagnosis (yes, no) was the secondary outcome. The ICD-9$C M$ codes associated with noxious substances affecting infants and breastfeeding children (760.7x) were captured to identify the type and magnitude of exposure. These diagnoses represent exposure to narcotics, including heroin and prescription opioid analgesics (760.72), hallucinogens (760.73), and cocaine (760.75).

\section{Descriptive Variables}

Descriptive patient-level characteristics associated with substance use and obstetric/neonatal health outcomes were analyzed. These variables included the newborns' gender, hospital length of stay, clinical conditions, in utero substance exposure, and mothers' 
insurance type. Seizures, adverse respiratory symptoms, and feeding difficulties are clinical conditions associated with NAS diagnosis.[11, 12, 89] Diagnoses and respective $I C D-9-C M$ codes, seizures (779.0, 780.3), respiratory symptoms (769.x, 770.x), and feeding difficulties (779.3), were coded to better describe the sample. Mothers' insurance type was a 4 category variable that encompassed Medicaid, Medicare, PEIA (WV Public Employees Insurance Agency), and Other (e.g., commercial companies and self-payers). In addition to the outcome, neonatal substance exposure codes (i.e., ICD-9-CM 760.72, 760.73, and 760.75) were described within the presence of a NAS diagnosis. These exposures are presented as separate binary variables (i.e., diagnosed versus not diagnosed), as newborns could have polysubstance exposure.

\section{Statistical Analysis}

NAS IRs were calculated using the number of NAS diagnoses as the numerator and respective number of live births in the denominator and multiplied by 1,000 to obtain IRs per 1,000 live births by region and year. This measure of infant morbidity captures newly occurring NAS diagnoses, which exhibits short duration and occurs only once during infancy.[90] Therefore, each newborn's person time at risk was equal to 1 unit (i.e., the number of annual live births).

The outcome was visually demonstrated via annual comparative WV region maps utilizing ArcGIS 10.2.[91] NAS IRs were stratified, in order to be representative of the SAMHSA 6 WV sub-state regions (see Figure 2.1).[71] Stratified by birthing hospitals, the hospital-level NAS IRs were represented by the diameter of circles on the GIS regional map (see Figure 2.2). 
To assess overall differences in proportions of newborns with and without NAS by year, the Mantel-Haenszel Chi-square $\left(\chi^{2} \mathrm{MH}\right)$ test was stratified by both region and hospital.[72] Additionally, the Cochran-Armitage test $(Z)$ for trend was utilized to test for linear trends in the proportion of NAS diagnosis from 2007 to 2013. Differences among delivery characteristics (i.e., neonatal length of stay, insurance type, and clinical diagnoses) and NAS diagnosis were tested using the Pearson Chi-square $\left(\chi^{2}\right)$ test for categorical characteristics and the Wilcoxon RankedSum test (z) for continuous characteristics. An a priori two-sided type 1 error of 5\% was considered statistically significant. Statistical analyses were conducted using SAS® 9.4 (SAS Institute Inc., Cary, NC).

\section{Ethics Statement}

Approval of the current study was obtained from the Institutional Review Board of the primary author's academic institution (Protocol \# 1405305752).

\subsection{Results}

\section{NAS and Exposure}

Overall, the current study analyzed 119,605 live births with 1,974 NAS diagnoses (i.e., a total of 16.5 per 1,000 live births) (see Figure 2.2). With regards to rates over time, between 2007 and 2013 the statewide rate of newborns diagnosed with NAS significantly increased from 7.74 to 31.57 per 1,000 live births per year $(\mathrm{Z}:-19.10, P<.0001)$. There were statistically significant differences in the magnitude of the trend in NAS IR by region; region 1 (Z: $-4.73, P<$ $.0001)$, region $3(\mathrm{Z}:-2.41, P=.016)$, region $4(\mathrm{Z}:-10.51, P<.0001)$, region $5(\mathrm{Z}:-8.00, P<$ $.0001)$, and region $6(\mathrm{Z}:-18.77, P<.0001)$ significantly increased over time. In 2013, the southeastern region of the state (i.e., region 5) had the highest NAS IR of 48.76 per 1,000 live births. There was no significant trend of NAS diagnoses in region $2(\mathrm{Z}:-0.002, P=.999)$. After 
adjusting for regional variation, there was an overall significant increase in NAS diagnoses from 2007 to $2013\left(\chi^{2}\right.$ MH=429.78, 6 d.f., $\left.P<.0001\right)$.

Between 2007 and 2013, the statewide rate of newborns diagnosed with neonatal substance exposure per 1,000 live births significantly increased from 1.89 to 8.09 for narcotics (Z: -9.56, $P<.0001$ ) (see Figure 2.3). Region-specific narcotic exposed diagnoses significantly increased over time in regions 1 (Z: $-5.30, P<.0001), 2$ (Z: $-4.24, P<.0001), 3$ (Z: $-2.02, P=$ $.04), 4(\mathrm{Z}:-8.46, P<.0001)$, and 6 (Z: $-2.52, P=.01)$. Neonatal exposure diagnoses did not differ over time in region $5(\mathrm{Z}: 0.84, P=.40)$. After controlling for region differences, the NAS IR trend was statistically significant $\left(\chi^{2} \mathrm{MH}=99.01,6\right.$ d.f., $\left.P<.0001\right)$.

During this time period the diagnosis of cocaine exposure decreased $(\mathrm{Z}: 3.62, P=.0003$ ) from 1.77 to 0.74 per 1,000 live births. Region-specific cocaine exposed diagnoses significantly decreased over time in regions $5(\mathrm{Z}: 2.49, P=.013)$ and $6(\mathrm{Z}: 3.02, P=.003)$. These diagnoses did not significantly decrease between 2007 and 2013 for regions 1 (Z: $0.27, P=.786), 2$ (Z: 1.20, $P=.229), 3(\mathrm{Z}: 1.46, P=.144)$, and $4(\mathrm{Z}: 0.733, P=.4636)$. After controlling for regional variation, cocaine diagnosed exposure decreased over time $\left(\chi^{2} \mathrm{MH}=17.77,6\right.$ d.f., $\left.P=.0007\right)$. Diagnosis of hallucinogenic agent exposure did not significantly change over time $(\mathrm{Z}:-1.61, P=$ $.11)$.

Controlling for year, the amount of NAS cases differed significantly by birthing hospital (n=30, $\chi^{2}{ }_{\text {MH }}=906.67,29$ d.f., $P<.0001$ ). Between 2007 and 2013, the average hospital rate of NAS diagnoses ranged from zero to 36.76 per 1,000 live births. During the study period, the 3 level III neonatal intensive-care units (NICUs) in WV delivered the following number of infants diagnosed with NAS: 239 (1.2\%), 150 (2.04\%), and 373 (2.95\%). 


\section{Characteristics by NAS Diagnosis}

Infants with a primary or secondary NAS diagnosis were more likely to exhibit respiratory issues $\left(\chi^{2}: 366.01,1\right.$ d.f., $\left.P<.0001\right)$, feeding difficulties $\left(\chi^{2}: 95.38,1\right.$ d.f., $\left.P<.0001\right)$, and seizures $\left(\chi^{2}: 92.50,1\right.$ d.f., exact $\left.P<.0001\right)$, compared to newborns without a NAS diagnosis. These babies were also statistically more likely to be diagnosed with narcotics $\left(\chi^{2}: 903.56,1\right.$ d.f., exact $P<.0001)$, hallucinogens (v: $11.77,1$ d.f., exact $P=.0156)$, and cocaine $\left(\chi^{2:} 113.53,1\right.$ d.f., exact $P<.0001)$ exposures than newborns without NAS. Comparatively, newborns diagnosed with NAS had a longer length of stay (z: $57.77,1$ d.f., $P<.0001)$, and were more likely to have Medicaid insurance $\left(\chi^{2}: 753.01,3\right.$ d.f., $\left.P<.0001\right)$ (see Table 2.1$)$.

\subsection{Discussion}

\section{NAS and Exposure}

The current study was the first to assess the incidence rate (IR) of NAS throughout the predominantly rural state of WV. Between 2007 and 2013, the statewide rate of NAS diagnosis increased over 4-fold. Although the upward trend was expected, the increase of NAS rates over time was markedly higher in WV compared to national estimates.[16, 17] In 2009, WV's NAS IR was 3.5 times as high as nationwide rates (3.4 vs. 11.8 NAS per 1,000 live births).[17] The same year, Ohio reported a NAS rate of about 5 cases per 1,000 live births and Vermont found a NAS and opioid exposure rate of 24.2 per 1,000 live births.[92, 93] Vermont's rate was likely higher because they utilized a different case definition (i.e., all opioid exposed infants and/or infants diagnosed with NAS were considered cases).[92] In 2010, WV's NAS IR was over 3 times as high as nationwide estimates (14.7 vs. 4.8 NAS per 1,000 live births).[16] In 2011, WV's statewide NAS IR was 16.9 per 1,000 live births, which was higher than the national average (3.4) and various state-specific rates in Tennessee (8.5), Kentucky (13.2), and Florida 
(7.52) in the same year.[16, 94] In 2012, WV's NAS IR was 3.7 times higher than nationwide rates (5.8 vs. 21.4 NAS per 1,000 live births).[16]

Between 2007 and 2013, the current study showed the rate of neonatal substance exposure diagnosis increased over 4-fold for narcotics while neonatal cocaine exposure decreased over 2-fold and hallucinogens remained stable. Further examination of a potential shift in maternal drug use is indicated. Although national estimates of neonatal substance exposure via the $I C D-9-C M$ diagnostic codes $760.7 x$ are not currently available, Ohio reported similar patterns to WV between 2004 and 2011 of neonatal narcotic, cocaine, and hallucinogen substance exposure.[93] In addition, nationwide data during this time period showed that opioid-related overdose deaths and substance abuse treatment admission discharges vastly increased, while these estimates decreased for cocaine-related deaths and treatment admissions.[95, 96] A drastic increase in the environmental availability of prescription narcotics (i.e., increased prescriptions written and dispensed, greater social acceptability for non-medical use, and pharmaceutical marketing) could explain the increase in neonatal opioid and other noxious substance exposure resulting in the quadrupled NAS rates.

\section{Geographic and Time Trend Variations}

In the present study, NAS rates differed by WV residential sub-state SAMSHA regions, with the highest occurring in the southeastern part of the state. This finding is congruent with other data, as age adjusted death rates due to drug poisoning per 100,000 WV residents was the highest in the south between 1999 to 2009.[97] In addition, other characteristics associated with substance use and obstetric/neonatal health outcomes were found to be higher among residents from the respective area, compared to the rest of the state. According to the $2010 \mathrm{WV}$ Vital Statistics, the highest percent of low birthweight babies, mothers less than 18 years of age at 
delivery, no prenatal care, and congenital anomalies occurred among residents from the southeastern regions of WV.[77] This area also had the highest prevalence of any mental illness in the past year (18 or older), nonmedical use of pain relievers (12 or older), residents without a high school degree, percent of drug/narcotic-related arrest, and lowest weighted average median household income.[71, 98, 99]

Compounding the described challenges, treatment facilities are unevenly available throughout WV. Throughout the study period, there were $9 \mathrm{WV}$ federally funded (methadone or buprenorphine) opioid maintenance therapy (OMT) facilities that accepted pregnant women for the recommended OMT treatment, of which only 1 was located in the southeastern area of WV (see Figure 3.1).[100] Although OMT can result in NAS, the therapy is preferred over continued illegal substance use in order to improve obstetric and neonatal outcomes via decreased maternal illicit behaviors, improved prenatal care, and eliminated acute intoxication and withdrawal during pregnancy.[89, 101] Therefore, higher regional occurrence of obstetric and neonatal health outcomes could be linked to the lack of available treatment options for this vulnerable population.[101, 102]

An examination of time trends by region showed that incidence of NAS rates first rose in the Eastern Panhandle and during subsequent years rose throughout WV, with the highest IR in the southern regions of the state. The high NAS rate in the Eastern Panhandle at the beginning of the study period (i.e., 2007) may explain why NAS IR trends were not statistically significant in region 2. Aside from the inherent risk factors mentioned above, this trend could have resulted from potential drug trafficking routes and increased medical awareness. The Office of National Drug Control Policy has identified 11 southern WV counties (9 located in region 6 and 2 in region 5) as high-intensity drug trafficking areas, due to the Appalachian drug abuse 
problem.[103] Geographically, US 19 corridor is also a high-intensity drug trafficking area because of the flow of prescription pills from Florida into the Appalachian area.[104] In addition, WV is located in the middle of drug trafficking routes on major highways connecting the west and southwest regions to the profitable East Coast markets.[105]

It is also likely that a significant increase in medical awareness has led to a higher rate of NAS diagnoses over time. There could be more accurate diagnosis in areas around major medical centers and hospitals located on the Ohio, Maryland, and Virginia borders. Although heightened provider awareness of NAS and its signs/symptoms could contribute to the increasing trend, the opioid epidemic documented in the literature, along with the current study finding of increased neonatal substance exposure, makes this possibility less likely.

Along with regional differences, NAS IR varied by hospital. High risk patients are more likely to deliver at the closest hospital capable of dealing with fetal and/or maternal complications. During the study period, the level III NICU hospitals, those capable of treating NAS diagnosed infants, had comparatively high NAS cases per 1,000 live births. The top 11 hospitals with average NAS IRs above 20 per 1,000 live births were located in the southeastern regions of the state (i.e., in regions 2,5 , and 6), resulting in more accessibility to patients residing in this area.

There was no clear pattern of regional differences among diagnosed neonatal substance use exposure. In addition, NAS rates were greater than substance exposure rates, thus they did not mirror each other. These findings indicate WV greatly underutilizes neonatal substance exposure codes (i.e., the 760.7x ICD-9-CM codes). NAS-related reporting and surveillance would be improved if practitioners diagnosed every NAS infant with respective exposure codes and if policy recommendations included adding NAS to WV's list of reportable diseases, 
implemented an interoperable insurance/hospital system, and created a statewide standardized surveillance system to track NAS. In 2013, Tennessee was the first state to establish a successful statewide real-time tracking surveillance system for NAS.[106] In the first year, they identified over 900 newborns with NAS, two-thirds originating from mothers who were legally consuming prescription medications while pregnant and most of whom resided in Appalachian counties.

\section{NAS Associated Impact}

The significant associations between NAS and neonatal clinical conditions (i.e., respiratory issues, feeding difficulties, and seizures), as well as NAS and substance exposure (i.e., narcotics, hallucinogens, and cocaine) are consistent with existing research.[11, 92, 107] As expected, infants diagnosed with NAS were also more likely to be covered by Medicaid and have a longer hospital length of stay.[17, 107] The average hospital length of stay for NAS infants in WV was 12.7 days, shorter than the average national estimate of 16 days. In contrast, the WV average length of stay for newborns not diagnosed with NAS was 2.86 days, equivalent to the US average of 3 days.[17]

As highlighted by associated neonatal comorbidity and longer hospital length of stay, the impact of NAS to WV is substantial. Addressing this high cost and medical resource intensive public health issue involves a multi-faceted approach to policy, interventions, and evaluation of systematic efforts. Rural pregnant women with substance use disorders have unique treatment needs.[82] Future research is needed to identify optional substance use treatment and potential barriers to treatment access for this population.

\section{Limitations}

There are inherent limitations to using hospital discharge data. The HCA-UB data was created for payment use, therefore it is restrictive in its use to research a statewide health 
condition. Hospital charges might not translate to actual costs because charges are often negotiated by payers and costs can be separated via mother and infant claims, thus a cost analysis was not conducted in the current study. In addition, hospital billing practices could change over time. Overall, population-based surveillance is dependent on accurate and homogenous statewide coding from documenting a condition in the medical records to being coded in the discharge abstract. Furthermore, hospital billing data may underreport the diagnosis of NAS and neonatal substance exposure, especially when not all newborns are drug screened and exposure status is based on maternal self-report.[17, 92, 109]

Statewide identifiable data were unavailable, therefore hospital admissions/discharges rather than individual patients were the units of observation. Although unlikely, NAS discharges may result in duplication when the same patient (i.e., a newborn) has multiple hospital admissions. This would potentially underestimate NAS rates because it could increase the total population (i.e., denominator), while the NAS cases (numerator) would remain constant as this condition is likely to be diagnosed at the first hospital admission. These potential problems are driven from the study design and data source, thus solutions are limited and constraints may be a limitation of the data. Although data limitations exist, the HCA-UB is retrospectively the best approach to obtaining a statewide NAS rate of WV residents because it is the only data source that currently captures all insurance types. Strictly using Medicaid data would overestimate NAS rates, as it would mostly capture the at-risk population.[109]

\section{Conclusion}

The present study showed that in a state where over half of the residents live in rural areas[76], newborns with NAS were more likely to present other clinical conditions, experience longer hospital length of stay, and be insured by Medicaid. In addition, WV statewide neonatal 
diagnosis rates of both NAS and narcotic exposure have markedly increased. Narcotic substances affecting fetuses or infants via placenta or breastmilk were diagnosed less often than NAS, indicating underreporting of this neonatal exposure diagnosis. This finding is important because in practice the ICD-9-CM NAS diagnostic code is not broken down by drug-specific causes and therefore these neonatal exposure codes should be utilized in order to examine drug-specific trends. Surveillance epidemiologists should take this finding into consideration when studying neonatal substance exposures.

The current study provides further public health justification to increase efforts aimed at reversing the NAS-associated burden within rural areas. Public health interventions or treatment programs for this vulnerable population are especially important in the southeastern region of WV. West Virginia providers and medical coders need to better utilize the neonatal substance exposure codes (ICD-9-CM 760.7x). In order to eliminate inherent limitations related to the currently available hospital discharge data, the state needs to provide standardized patient-level identified data for future public health research and quality improvement initiatives. 


\section{Chapter 3}

\section{Exposure to substance use in utero, risk of neonatal abstinence syndrome, and antenatal correlates: A retrospective medical chart review}

\section{Abstract}

Objective: Although opioid in utero substance exposure is the primary cause of neonatal abstinence syndrome (NAS), other non-opioid substances have been linked to NAS diagnoses. The risk of NAS and NAS requiring pharmacological treatment among non-opioid exposures is unknown. Method: A retrospective medical chart review was analyzed for 476 mother-infant antenatal exposed dyads who delivered between January $1^{\text {st }} 2009$ and March $3^{\text {rd }} 20014$, with 137 NAS diagnoses (ICD-9-CM 779.5), and 75 diagnoses that required pharmacological treatment. Risk of NAS/NAS requiring treatment and odds of NAS, controlling for potential confounders via a logistic regression, were observed among drug class-specific exposure groups operationalized via neonatal meconium and umbilical cord tissue drug screens. Results: The absolute risk of NAS was $29 \%$ and risk of NAS requiring treatment was $54 \%$. Variations existed between drug class exposures, specifically opioid exposure (poly and single) had the highest risk of NAS and sedative-only exposure had the highest risk of NAS requiring treatment. After controlling for potential biopsychosocial confounders, odds of NAS was significantly higher for opioid exposures [single: $\mathrm{OR}=3.72,95 \%$ CI: $1.54,9.98$, poly: $\mathrm{OR}=3.52,95 \%$ CI: $1.13,10.98$ ]. Number of prenatal care visits and maternal opioid maintenance therapy enrollment were independent risk factors for NAS diagnosis. Conclusion: A NAS diagnosis most commonly occurred with opioid exposures, though risk among non-opioid exposures ranged from $11 \%$ to $33 \%$. There are no drug-specific diagnostic criteria or treatment protocols for neonatal substance withdrawal. Future examination of non-opioid based NAS protocols and studies regarding NAS severity with sedative in utero exposures are warranted. 


\subsection{Introduction}

Intrauterine exposure, to nearly any psychoactive licit or illicit substance, can lead to neonatal drug-related adverse outcomes or inadequate fetal growth which are major factors in infant disability, and, in severe cases, mortality.[22, 110] Psychoactive drugs readily transfer from the mother to her neonate through the trans-placental passage, which can cause early disrupted fetal programming and developmental processes via various pathophysiologic pathways.[22, 110] In 2009, a multi-hospital study in WV drug tested neonatal umbilical cord tissue specimens and found $19.2 \%$ of neonates were exposed to any substances (roughly 1 in 5 ). [4] Specifically, $5.4 \%$ and $1.8 \%$ of the total population tested positive of opioids and methadone. The same year, nationally representative data indicated $0.56 \%$ of mothers used and/or were dependent on methadone or any other opiate analgesic during pregnancy. [17] Although substance use among pregnant women is prevalent nationwide, WV's estimates outnumber the national averages indicating a significant public health issue.[1, 17] Due to the increase in incidence and geographic distribution of substance use in pregnancy, neonatal abstinence syndrome (NAS) is an increasingly concerning public health issue.

NAS is a constellation of central nervous system, metabolic vasomotor, respiratory, and gastrointestinal disturbances a neonate experiences resulting from drug withdrawal following delivery.[11, 12] Between 2000 and 2012, the nationwide incidence of NAS increased nearly $500 \%$ from 1.2 to 5.8 per 1,000 hospital births per year.[16, 17] In 2009, it was estimated that each hour a baby was born diagnosed with drug withdrawal in the United States.[17] Along with the health ramifications of NAS, associated fiscal consequences and strained health care resources result from treating this diagnosis. In 2009 the US public health and medical cost resulting from babies born with drug withdrawal was between 70.6 and 112.6 million 
dollars.[18] The average length of stay in hospital care for babies born from opioid dependent women is 16 days, which is dramatically longer than the average of 3 days for healthy neonates.[17]

Higher financial impact and longer neonatal hospital length of stay reflect the overall severity of NAS. NAS severity is dependent on numerous factors that include poly-drug use, gestational age of substance use, maternal smoking, psychiatric medications, other obstetric outcomes, and genetic factors.[112, 113] If neonates have severe enough NAS they will be pharmacologically treated with an opiate or opioid based medication.[11]

NAS most commonly occurs among neonates exposed to licit or illicit opioids during pregnancy, although other substances can induce similar signs and symptoms of withdrawal and therefore results in NAS diagnosis.[17, 22] Although a lack of literature exists regarding NAS and non-opioid exposures, especially among a general substance using maternal population, NAS symptomatology has been linked to alcohol [23-25], nicotine [26-29], cocaine[30-32], phencyclidine (or PCP) [33], methamphetamines [34], benzodiazepines[35, 36], and other psychotropic medications[37-40].

Identification of NAS diagnoses with non-opioid in utero exposures are problematic because generally there are no drug-specific diagnostic criteria for withdrawal nor are there drugspecific treatments for nonopioid-exposed neonates. The scoring system utilized to diagnose, assess severity, and determine treatment protocols is based off opioid withdrawal symptoms of term babies, when in fact drug-specific neonatal withdrawal is likely to differ based on the substance of exposure and the gestational age of the neonate.[22, 41] In addition, the most common first-line treatment of NAS is morphine or methadone.[11] Opioids might not be the choice of treatment in non-opioid exposed neonates, especially with the rising concern of 
iatrogenic NAS. Iatrogenic or hospital-induced NAS is when the syndrome is inadvertently induced via medical treatment, as opposed in antepartum substance exposure.

The purpose of the current study is to evaluate the risk of both NAS and NAS-requiring pharmacological treatment among neonates of substance-using women, and to determine whether cases differ based on drug class-specific exposures. In addition, the odds of NAS were estimated while controlling for potential confounders.

\subsection{Methods}

We conducted a retrospective cohort study of mother-infant dyads exposed to antenatal substance use. Specifically, a retrospective electronic medical chart review was conducted at a major birthing center located in north-central WV between January 1, 2009 and March 3, 2014. Ethical approval for the current study was obtained from the Institutional Review Board at the academic institution of the authors.

The following inclusion and exclusion criteria were adopted in order to assemble a retrospective cohort of neonates who were exposed to substances in utero. Women who had an active drug use diagnosis at delivery (ICD-9-CM: 303.9x, 304.x, 305.x, 648.31, 648.32, 649.01, 649.02), any positive urine drug screen in the nine months prior to delivery, were prescribed buprenorphine or methadone at delivery admission prior to giving birth (eRx codes: 4952, 4953, 4954, 10546, 87402, 87403, 87408, 87409, 135736, 190788, 201165, 201166, 1000152, 1000430, 1000431, and 1000555), or who delivered a neonate diagnosed with NAS (ICD-9-CM: 779.5) were included in the study. Electronic prescribing codes for buprenorphine or methadone during the delivery admission are unique indicators of a pregnant woman currently enrolled in an opioid maintenance therapy (OMT).[61] Women younger than 18 years of age at delivery and neonates younger than 28 weeks gestational age were not included in the study population. 
Mother-infant dyads were created by matching the woman's delivery admission with the neonate's hospital admission. The sample was limited to the first singleton live birth (ICD-9-CM: V27.0) of women who met the inclusion criteria.

Mother-infant dyads were excluded if they did not deliver at the study hospital ( $\mathrm{n}=5)$, the neonatal electronic medical records weren't transferred in its entirety from the old to the new electronic health record system at the study hospital $(n=2)$, the neonate expired during the same delivery admission $(n=8)$, or if the neonate was deemed to have iatrogenic NAS $(n=15)$. Neonates at risk for iatrogenic NAS are those born with an intraventricular hemorrhage (ICD-9$C M$ 772.1x), periventricular leukomalacia (779.7), necrotizing enterocolitis (777.5x), spontaneous intestinal perforation (777.6), or bronchopulmonary dysplasia (770.7).[17] The etiology of withdrawal is different between hospital-induced and intrauterine exposed neonates; therefore, neonates at risk for iatrogenic NAS were excluded from the study. A total of 29 mother-infant dyads were excluded from the study, as one neonate expired during the hospital admission and met the criteria for iatrogenic NAS.

After patients were selected for study inclusion and easily accessible clinical parameters were collected via a hospital decision support analyst, medical charts were critically reviewed. Medical notes in each patients' charts were read to determine the presence of biopsychosocial variables of interest. Manually-abstracted data were entered and stored in a HIPAA compliant, web-based, electronic data capture application called Research Electronic Data Capture (REDCap). ICD-10-CM codes were not in use during the study period; therefore, all diagnostic indictors where derived from $I C D-9-C M$ codes. 


\section{Exposure}

In utero substance exposure was defined as mutually-exclusive drug class grouping via non-invasive umbilical cord tissue and meconium biological drug screens conducted at delivery. To determine absolute risk of NAS diagnosis and NAS requiring pharmacological treatment, a seven category drug class-specific exposure variable was created that included opioids-, cannabis- stimulants-, and sedatives-only, poly-substances with opioids, poly-substances without opioids, and non-positive test results. Non-positive results do not exclude the possibility of prenatal substance use; they simply indicate that the drug or metabolite in the specimen did not meet the concentration cutoff required for a positive screen (see Appendix E). In addition, exposure to alcohol, nicotine, and caffeine was not determined as these substances are not included in the meconium and umbilical cord tissue drug screen panel.

To then estimate the odds of NAS diagnosis among neonates of substance using women, a five category drug exposure variable was created that includes opioids-, cannabinoids-only, poly-substances with opioids, other, and non-positive. 'Other' includes neonates singularly or poly-exposed to drug classes other than opioids and cannabinoids. Non-positive results are defined in the paragraph above. Specific drugs that are categorized in the five exposure groups are outlined in Table 3.1. None of the neonates in the sample tested positive (meconium or umbilical cord tissue) for phencyclidine (i.e., PCP), and thus it was not included in the drug grouping.

Neonatal specimens were collected for medical purposes only and sent for analysis to Associated Regional and University Pathologists (ARUP) laboratories in Salt Lake City, Utah. Universal umbilical cord tissue drug screens have been initiated on all neonates at the study birthing center from since March 12013 . Prior to the initiation of universal cord-tissue drug 
screens, meconium drug screens were conducted on all neonates suspected of substance exposure (defined in the outcome section above). Meconium is the first stool that the neonate passes that begins to form at 12-16 weeks gestation, prior to the appearance of breastmilk- or formula-based stool.[114] Therefore, meconium acts as a reservoir for drug and metabolite compounds and is a reliable specimen in which to assess in utero substance exposure.[114] Umbilical cord tissue specimen is thought to detect substance exposure during the same range of gestational ages as meconium. In addition, umbilical cord tissue is viewed as an alternative to meconium drug screening because both have similar drug detection abilities and have an agreement above $90 \%$ for amphetamine, opiates cocaine, cannabinoid, and phencyclidine tests.[49, 50]

Although screening umbilical cord tissue is a more recent technique and less is known about the disposition of drugs in the specimen, clinicians often prefer its use over meconium. Meconium collection is often delayed compared to umbilical cord tissue collection because it typically takes up to three days after birth to pass, or often longer among premature neonates.[115, 116] Additional drawbacks to meconium testing verses umbilical cord tissue testing are availability and compromised samples. Meconium may not be available if passed in utero or during birth and it can be compromised if it is diluted with other substances (e.g., urine) or if there is not enough specimen for confirmation testing.[45] Both meconium and umbilical cord tissue testing are preferred over maternal and neonatal urine drug screens, as they have a longer detection window.[45]

Meconium samples (at least 4 grams) were screened via the hospital's testing protocol by enzyme-linked immunosorbent assay (ELISA) to identify the drug class (i.e., cannabinoids, cocaine, opiates, phencyclidine, amphetamines, barbiturates, methadone, benzodiazepines, and propoxyphene). Any positive results were followed by confirmation of a fully validated gas 
chromatography-mass spectrometry or liquid chromatography-tandem mass spectrometry.[117]

Drug classes screened via umbilical cord tissue specimens included opioids, stimulants, sedatives-hypnotics, cannabinoids, and phencyclidine. Specimens of umbilical cord tissue (at least 6 inches) were tested via a 'gold standard' of liquid chromatography-time of flight mass spectrometry and ELISA.[118, 119] Sensitivity and specificity of the ELISA compared to the mass spectrometric method were $91 \%$ and $98 \%$, respectively.[120]

\section{Outcome}

The outcome of interest for this study was the incidence of neonatal abstinence syndrome (NAS) among substance-exposed infants. The outcome was defined as 'present' if a neonate had a primary or secondary diagnosis of NAS (ICD-9-CM code 779.5: Drug withdrawal syndrome in newborn) and 'absent' if there was not a NAS diagnosis in the neonate's medical record. The withdrawal diagnosis does not specify the drug exposure that resulted in the neonatal withdrawal.

NAS diagnosis and severity was initially assessed via the modified Finnegan Scale.[41] Neonates exposed or suspected of exposure to in utero substances were evaluated prior to feeding, every 2 to 4 hours after birth. Suspected neonates are those that are born to mothers with a history of drug use, minimal to no prenatal care, and unexplained placental abruption or premature labor, and are born with unexplained neurological complications, unexpected intrauterine growth retardation, and evidence of intoxication (e.g., positive maternal urine drug screen during pregnancy) and/or NAS signs and symptoms.[48] NAS was diagnosed when a neonate scored $\geq 8$ during two consecutive screenings. Non-pharmacologic treatment occurred at the onset of all NAS diagnoses (i.e., kangaroo care, swaddling, high caloric diet, and a lowstimuli environment). If NAS was more severe (i.e., neonates with a score of $\geq 12$ or greater on 
three consecutive occasions or a combined score of $\geq 28$ ) than pharmacological treatment of morphine sulfate drops were administered.

\section{Covariates}

To calculate the least-biased estimate of the effect of in utero substance exposure on the incidence of NAS, confounding was explored and controlled in the analysis. Biologic, psychological, and socioeconomic factors of the women and/or neonates associated with NAS and maternal substance use were specified using the biopsychosocial model as a theoretical framework as shown in Figure 3.1.[70] This model aims to assess health-related outcomes via a holistic multi-faceted approach.

\section{Biological}

Biological factors likely to influence the substance to which the fetus was exposed and neonatal expression of drug withdrawal include maternal age, nulliparous status, current gravidity, pre-pregnancy maternal body max index, delivery type (cesarean or vaginal), neonatal gestational age (weeks), and neonatal growth parameters of head circumference (centimeters), length (centimeters), and weight (grams) at delivery.[121-123] Delivery type was categorized as a binary variable of cesarean or vaginal delivery.

\section{Psychological}

The aggregated presence of maternal psychological-related diagnostic codes given at labor and delivery were utilized as a psychological factor in the biopsychosocial model.[124] This variable was categorized as 'present' if the mother was diagnosed with a mood disorder (IDC9-CM: 293.83, 296.90, 296.99), anxiety disorder (293.84, 300.00, 300.02), schizophrenia (295.30, 295.62, 295.90), major depressive disorder (296.20, 296.23, 296.25, 296.30, 296.32, 296.33, 296.36), bipolar (296.40, 296.42, 296.50, 296.53, 296.60, 296.7, 296.80, 296.89), 
unspecified psychosis (298.9), panic disorders (300.01), conversion disorder (300.11), agoraphobia (300.21), obsessive-compulsive disorders (300.3), dysthymic disorder (300.4), antisocial personality disorder (301.7), borderline personality disorder (301.83), other isolated or specific phobias (300.29), unspecified nonpsychotic mental or personality disorders (300.9, 301.9), and other pervasive developmental disorders (299.80).

\section{Socioeconomic}

Socioeconomic factors included as covariates consisted of maternal years of education at delivery, Medicaid insurance status and metropolitan residence.[125, 126] Medicaid insurance status was an artificially dichotomized variable of 'Medicaid' or 'other.' Living in a metropolitan area was defined via a patient's county of residence and the Office of Management and Budget's delineation.[127] A metropolitan area was defined as having at least one urbanized area (10,000 or more inhabitants) of 50,000 or more residents. Rural-Urban 2013 Continuum Codes were utilized to match each patient's county of residence to the appropriate categorization of metropolitan or nonmetropolitan status.

\section{Biopsychosocial Unions}

Covariates operationalized as biopsychological included number of spontaneous abortions, maternal history of substance use or mental health treatment, maternal opioid maintenance therapy enrollment, and history of chronic pain.[126, 128] Spontaneous abortions were defined as a non-therapeutic miscarriage before 20 weeks gestational age. Maternal opioid maintenance therapy during pregnancy was captured as a binary (yes/no) variable indicating whether the women were enrolled in buprenorphine or methadone maintenance treatment. History of substance use or mental health treatment and chronic pain are both binary variables (yes/no). The number of prenatal care visits during pregnancy was categorized as a bio- 
socioeconomic variable.[125] Psycho-socioeconomic variables include marital status, whether the patient's family visited the hospital during labor and delivery admission (yes/no), reported familial support (yes/no), documented signs of physical abuse at hospital admission (yes/no).[123, 125, 129] Marital status was categorized as married, single, or other; with other including mothers who are divorced, legally separated, or widowed. Maternal use of selective serotonin reuptake inhibitors (SSRIs) and the self-reported number of cigarette packs smoked per day were represented by all three areas of the biopsychosocial model.[130] Packs of cigarettes smoked per day was a continuous number that ranged between zero to five packs.

\section{Statistical Analysis}

The incidence of NAS and NAS requiring pharmacological treatment was calculated and the number of cases, corresponding risk, and 95\% confidence intervals $(\mathrm{CI})$ are reported by drug exposure groups. To determine significant differences between substance groups, the Chi-square test was conducted with the level of significance at alpha less than or equal to .05 .

Descriptive statistics were computed and reported as frequencies and percentages for categorical variables and as means and standard deviations for continuous variables (or median and interquartile ranges for non-normally distributed data). Maternal and neonatal characteristics by NAS diagnosis were assessed via Cochran-Armitage trend test, Fisher's exact, or Chi-square test for categorical variables and student's t-test and Wilcoxon rank-sum test for continuous measures.

Potential confounders were identified via the change-in-estimate method. That is, if covariates changed the effect estimate (i.e., odds ratio) of the neonatal in utero substance exposure variable by 10 or more percent it was included in the regression model.[73] The adjusted logistic regression model controlled for maternal history of chronic pain (referent was 
no history), history of drug/mental health treatment (referent was no history), number of prenatal care visits, cigarette packs per day, whether the mother's family visited her during the labor and delivery hospital admission (referent was no visit), whether the mother reported family support at hospital admission (referent was no support), and the mother's pre-pregnancy body mass index. All analyses were conducted using SAS 9.4 (SAS Institute Inc., Cary, North Carolina).

\subsection{Results}

Of the 543 mother-infant dyads who met the inclusion criteria, $476(88 \%)$ had a meconium or umbilical cord tissue drug screen conducted at delivery and were therefore included in the analysis. Of the neonates included in the analysis, 137 (29\%) were diagnosed with NAS, and of those diagnosed with NAS, 75 (55\%) were pharmacologically treated. Based on drug screen results, 110 (23\%) neonates were defined as opioid-only exposed, 94 (19.8\%) were defined as cannabis-only exposed, 75 (15.8\%) were defined as poly-substance including opioids exposed, 31 (77) were positive for 'other' drug exposure, and 166 (35.9\%) were nonpositive. Specifically, $9(1.9 \%)$ of the neonates categorized as 'other' exposure tested positive for stimulants-only, 15 (3.2\%) were sedatives-only, and 7 (1.5\%) were exposed to poly-substances without opioids.

\section{NAS and Pharmacological Treatment by Drug Class Exposure}

The risk of NAS was $29.2 \%$ and, of those diagnosed with NAS, the risk of being pharmacologically treated was 54\%. Categorized by drug class-specific exposures, opioid-only exposed neonates had an absolute risk of NAS of $45.5 \%$ and of those $54 \%$ were pharmacologically treated. Cannabis-, stimulant-, and sedative-only had an absolute risk of NAS of $12.8 \%, 11.1 \%$, and $33.3 \%$ and within the exposure categorization $33.3 \%$, and $80 \%$ were pharmacologically treated for NAS, respectively. Poly-substance with opioids exposure had an 
absolute NAS risk of $41.3 \%$ and of those diagnosed with NAS, $67.7 \%$ were pharmacologically treated. Poly-substance exposure without opioids had an absolute NAS risk of $28.6 \%$ and of those diagnosed with NAS, 50\% were pharmacologically treated. Neonates of substance-using mothers who did not screen positive had an absolute risk of NAS of $33.8 \%$ and of those diagnosed with NAS, $47.4 \%$ were pharmacologically treated.

\section{Maternal and Neonatal Characteristics}

The average maternal age was 26.1 years $(\mathrm{SD}=5.1)$, median years of education completed was 12 (IQR 11, 13), 8 (1.7\%) mothers exhibited signs of physical abuse, $79(17.3 \%)$ had a history of chronic pain, the median amount of packs of cigarettes smoked per day were 0.5 packs (IQR 0.2, 1.0), 60 (12.7\%) were prescribed SSRIs during pregnancy, and 100 (21.0\%) were diagnosed with a mental health condition. A majority of women were single $(304 ; 64.7 \%)$, insured by Medicaid (375; 78.8\%), lived in metropolitan defined areas $(256 ; 54.6 \%)$, had family visit them during the labor and delivery hospital admission $(374 ; 91.7 \%)$, were enrolled in opioid maintenance therapy during pregnancy $(209 ; 44 \%)$, and had a history of drug and/or mental health treatment $(268 ; 57.0 \%)$. About a third of the women were nulliparous $(37.2 \%)$, the median gravidity of $2(\mathrm{IQR}=2,4)$, and the median spontaneous abortions of $0(\mathrm{IQR}=0,1)$. Maternal characteristics of the delivery of interest included an average of $8.8(\mathrm{SD}=5.7)$ obstetrics/gynecology visits during pregnancy, mode of delivery (300 or 63\% vaginal and 176 or $37 \%$ cesarean $)$, and maternal pre-pregnancy BMI of $22.6(\mathrm{IQR}=19.7,27.5)$. The slight majority of neonates were male $(240 ; 50.4 \%)$ and neonatal growth parameters include: gestational age of 38.1 weeks $(\mathrm{IQR}=36.9,39.2)$, head width of 33 centimeters $(\mathrm{IQR}=31.5,34)$, length of 47.6 centimeters $(\mathrm{SD}=3.8)$, and weight of 2,816.7 grams $(\mathrm{SD}=620.8)$. 


\section{Prenatal Substance Exposure and NAS}

Neonates whose meconium or umbilical tissue tested positive for substances, who were born to women who had a history of chronic pain, a history of drug or mental health treatment, were enrolled in opioid maintenance therapy during pregnancy, and who were born to women who had fewer prenatal visits were significantly more likely to develop NAS than their counterparts.

Neonates exposed to opioids-only were nearly three times as likely [unadjusted $\mathrm{OR}=$ $2.81 ; 95 \%$ CI: $1.67,4.73]$ to be diagnoses with NAS compared to neonates not positively screened for drug exposure. Poly-substance with opiate in utero exposure and neonates with other substance exposure were $137 \%$ [unadjusted $\mathrm{OR}=2.37 ; 95 \%$ CI: $1.32,4.26$ ] and $17 \%$ [unadjusted OR $=1.17 ; 95 \%$ CI: $0.49,2.83$ ] more likely to be diagnoses with NAS than neonates not positively screened for drug exposure, respectively. Cannabis-only exposed neonates were $51 \%$ [unadjusted OR $=0.49 ; 95 \%$ CI: $0.24,0.99$ ] less likely to be diagnoses with NAS compared to neonates not positively screened for drug exposure.

When controlling for biopsychosocial factors, the association between in utero substance exposure and NAS persisted for opioid-only and poly-substance with opioid exposed neonates. The adjusted odds of NAS among opioid-only and poly-substance with opioid exposed neonates was $272 \%$ [OR $=3.72 ; 95 \%$ CI: $1.54,8.98]$ and $252 \%$ [OR $=3.52 ; 95 \%$ CI: $1.13,10.98]$ greater than the odds of NAS among neonates without a positive drug screen, respectively. Adjusted biopsychosocial factors significantly associated with the odds of NAS included maternal enrollment in opioid maintenance therapy at pregnancy and number of prenatal care visits. For every increase in number of prenatal care visits, the odds of delivering a neonate diagnosed with NAS decreased $8 \%$ [OR $=0.92 ; 95 \%$ CI: $0.86,0.99]$. Neonates of mothers enrolled in opioid 
maintenance therapy were more than six times [OR $=6.54$; $95 \% \mathrm{CI}: 2.30,18.57]$ as likely to be diagnosed with NAS compared to neonates born to mothers not enrolled

\subsection{Discussion}

Primary Findings

The study cohort of neonates who were exposed to substances in utero had a high absolute risk of NAS (29.2\%) and of those diagnosed most were pharmacologically treated (54.0\%). Risk of NAS significantly varied among drug exposure type. The highest risk occurred with opioids-only and poly-substances with opioids, and the lowest risk of occurred within the stimulants-only exposure group. Of the neonates diagnosed, the highest drug class exposure requiring treatment for NAS ranged from sedatives-only as the highest to cannabis-only and stimulants-only as the two lowest exposure groups. Only one neonate was diagnosed with NAS and had a stimulants-only exposure, therefore this risk statistic cannot be empirically evaluated.

After controlling for biopsychosocial covariates, neonates exposed to opioids-only and poly-substance with opioids were significantly more likely to be diagnosed with NAS. The covariates that remained significant in the fully adjusted model include number of prenatal care visits and maternal opioid maintenance therapy enrollment. Greater number of prenatal care visits was associated with a lower likelihood of a being diagnosed with NAS. This finding is expected as increased prenatal care is associated with improved maternal and neonatal health outcomes.[109, 131] Although increased screening will likely increase the chance of a neonate being identified and diagnosed, the benefit of prenatal care likely outweighs the potential influence of increased detection. As expected, neonates of mothers enrolled in OMT during pregnancy had a higher likelihood of being diagnosed with NAS. Women in OMT are given buprenorphine or methadone during pregnancy to stabilize their opioid dependency and 
eliminate antenatal withdrawal. In utero exposure to both buprenorphine and methadone can result in NAS.[132]

\section{Risk Comparisons with Other Studies}

Risk and severity of NAS differentiate between studies via the study population (e.g., only mothers enrolled in OMT or a general at-risk population) and operationalization of in utero substance exposure. Risk of NAS and NAS requiring pharmacological treatment typically are studied among infants of opioid dependent mothers and/or among women who filled a prescription of opioids during pregnancy. Between 1998 and 2009, 5.6\% of women who were prescribed narcotic pain medication for more than one month during pregnancy delivered at newborn diagnosed with NAS.[133] Medicaid data revealed that infants born to women who filled a prescription for the same length of time during pregnancy had a $2.4 \%$ risk of being diagnosed with NAS.[61] This risk increased when opioid prescriptions were filled within the last 90 days before delivery and in the presence of other risk factors, including a documented history of opioid drug misuse/dependence, history of alcohol/non-opioid misuse, use of other psychotropic medications in the third trimester, and a documented smoking history.

In addition to various study populations, risk of NAS and NAS requiring treatment differentiate between infants exposed to heroin throughout pregnancy verses those exposed to OMT. Among neonates exposed to heroin throughout gestation, $42 \%$ were diagnosed with NAS and 18\% required pharmacological treatment.[134] Another study reported 27\% of infants born to mothers on methadone maintenance therapy were diagnosed with NAS.[109] Additionally, in 2010 a randomized control trial compared the effectiveness between buprenorphine and methadone OMT among 175 opioid dependent pregnant women enrolled in therapy, with respect to neonatal growth parameters and NAS diagnosis, treatment, and length of stay indicators.[132] 
Results indicated both medications were acceptable OMT options and that the risk of NAS requiring treatment was $47 \%$ and $57 \%$ among infants born to mothers on buprenorphine and methadone maintenance therapy during pregnancy, respectively.[132]

There is a lack of research of NAS and NAS requiring pharmacological treatment among infants exposed to nonopioid substances in utero. One study assessed the risk of NAS among heroin only, cocaine-only, and opioid and cocaine exposed infants and found the risk to be $14 \%$, 6\%, and 35\%, respectively.[134] Another study assessed the risk NAS among infants born to mothers with an opioid prescription filled and who used non-opioid substances during pregnancy. Within this cohort, women who were also prescribed a psychotropic medication in the third trimester (i.e., sedatives) delivered infants with a $1.3 \%$ risk of NAS diagnosis. In addition, women who filled an opioid prescription and had a documented alcohol and/or nonopioid use disorder had a 3.1\% risk of delivering a NAS baby.[61] Another study that assessed risk of NAS requiring pharmacological treatment among a cohort of NAS diagnosed infants found the greatest risk to be among infants exposed to an opioid (i.e., methadone) and another nonopioid substance $(82.5 \%)$, followed by an opioid-only (80\%), a single non-opioid (68.3\%), and a poly nonopioid (50\%) in utero exposure.[62] Although no studies have comparatively examined the risk of NAS among sedative- and stimulant-only exposures, $30 \%$ of infants exposed to SSRIs during pregnancy were diagnosed with NAS and $4 \%$ of infants exposed to methamphetamine required pharmacological treatment for NAS.[34, 135].

Although the literature differs with respect to study population and in utero drug class exposure groups, the current study had a considerably higher risk of NAS among opioid exposed neonates compared to other studies with mother's who filled an opioid prescription during pregnancy (i.e., $46 \%$ vs. $2.4 \%$ to $5.6 \%$ ).[61, 133] This discrepancy is most likely due to 
differences in population characteristics. That is, the current study encompassed a more at-risk population than women who filled an opioid prescription antenatally. The current study had a comparable risk of NAS and risk of NAS requiring treatment among opioid-only exposed neonates compared to study populations of mothers' with an illicit and/or licit opioid dependency.[132, 136, 137]

With respect to the literature, our study differentiates via exposure groups linked to the highest risk of NAS requiring treatment. In the current study, the largest risk for pharmacological treatment is among sedative-only exposure and it was followed by opioid-only and polysubstance with opioid exposures. The comparative study had methadone-only, methadone and other, single non-methadone, and polysubstance non-methadone exposure groups with polysubstance and then single methadone exposures leading the pharmacological treatment risk. Discrepancies could have resulted from different categorization of substance exposure groups. According to our results, sedative-only exposures could have a lower occurrence, but result in more severe NAS. Selective serotonin re-uptake inhibitors are common sedatives that are linked to NAS diagnosis and pharmacological treatment of neonatal withdrawal and our study cohort had a relatively high prevalence rate of $13 \%$ of mothers who used SSRIs during pregnancy. This finding would be unearthed within the previous study, as sedative-only exposures would have been categorized in a less granular exposure group of single non-methadone substances.

\section{Clinical Implications}

Variation existed among drug class-specific exposure groups and risk of NAS diagnosis and NAS requiring pharmacological treatment. This variation indicates multiple substances can lead to a NAS diagnosis, not just opioids, and diagnoses should be more specific to represent the exposure that results in the withdrawal. Proper diagnosis of exposure is important to better treat 
patients and for research purposes of tracking substance use trends in this vulnerable motherinfant population.

In addition, we showed variation among the hierarchical ordering of exposures associated with risk between NAS diagnosis and severity of NAS. Opioid exposure (single or multiple substances) had the highest risk of NAS diagnoses, but was the second highest risk of NAS requiring treatment; following sedative-only exposure. Further examination of sedative in utero exposure resulting in more severe NAS, compared to other drug class-specific exposures is indicated.

Clinically, this difference raises questions regarding hospital protocols identification and treatment for newborns diagnosed with NAS. Regardless of the exposure, these infants are largely being diagnosed and treated with the same protocol. The modified Finnegan scoring system was created from opiate withdrawal signs and symptoms and it is the most common NAS scoring tool used in American. In addition, $83 \%$ of US clinicians use an opiate (usually methadone or morphine) as the first line of treatment for NAS.[138] Most cases of NAS are linked to in utero opioid exposure. Therefore, in many cases the neonates withdrawing from nonopioid substances are then given opioids as treatment in the NICU. This could be problematic, as iatrogenic NAS increased from $9 \%$ in 2003-2004 to $50 \%$ in 2010 to 2011 . among infants treated with morphine for postoperative, ventilated, or chronically ill conditions.[13] Therefore, future research should focus on the effectiveness of current standardized diagnostic and treatment opioid-based hospital protocols, in instances when there is a known non-opioid exposure status. In addition, phenobarbital and clonidine are utilized as second-line therapy options for NAS when first-line opioid based medications are ineffective.[139] Future research should explore the 
effectiveness of phenobarbital as a first-line treatment for sedative-exposed neonates with severe NAS.

In addition to outlining absolute NAS and NAS requiring treatment risks among varying in utero exposures, it is important to identify other significant maternal and obstetric factors that increase the offspring's likelihood of a NAS diagnosis. This information is helpful for clinicians to better identify at-risk neonates that could have been undetected from typical substance exposure screening methods (e.g., history of drug use, maternal intervening, and universal maternal urine drug screen). With the current screening techniques, withdrawal signs and symptoms could be mistaken as a 'fussy' baby and thus not be diagnosed and treated. In addition, withdrawing infants could be sent home prematurely without a NAS diagnosis, as signs and symptoms usually start 48-72 hours after birth.[140, 141]

\section{Strength and Limitations}

The current study benefited from utilization of biologic samples of the neonates to identify in utero substance exposure. Meconium and/or umbilical cord tissue drug screens with confirmatory testing following positive results are considered the most accurate and practical non-invasive tests compared to other screening methods, for example maternal and neonatal urine drug screens, hair sampling (results in higher false-positive results), and self-report from interviewing.[46, 68] Although utilization of meconium and umbilical cord tissue drug screening provided stronger evidence of exposure classification, there are limitations associated with this type of exposure assessment. For example, a non-positive drug screen result does not exclude the possibility that a mother used drugs during pregnancy because drug metabolites could be below the cut-off values for positive tests and detection could depend on unique characteristics of drug class-specific deposition in the meconium and/or UCT. In addition, certain substances associated 
with neonatal withdrawal are not screened in the meconium and UCT drug panels (i.e., alcohol, nicotine, and caffeine) and meconium and UCT results did not perfectly mirror each other, as meconium did not include buprenorphine while UCT. Detection of drugs depends on the extent and stability of maternal drug use and the pattern and frequency of drug(s) used by the mother cannot be determined by the aforementioned drug screens. Drugs administered during labor and delivery could also be detected within meconium and UCT screening, though this is not a strong limitation of the current study, as the cohort encompassed substance exposed neonates rather than neonates with a positive biological drug screen.

The selected patient sample is a strength of the study as it targets a cohort of neonates exposed to substances in utero. This criteria allows the study population to be more generalizable, because it does not restrict patients to only those with a positive drug screen or only mother-infants dyads with a NAS diagnosis. In addition, this generalizable population allows researchers to appropriately study multiple drug class-specific exposures related to NAS, which would not be accurately assessed via a population of women enrolled in opioid maintenance therapy or women who filled an opioid prescription during pregnancy. This is important, as the current study points out that nonopioid in utero substance exposure is also linked to NAS. Although broad, the inclusion criteria could still have resulted in undetected substance-using mothers. Therefore, neonates who met the criteria to be in the sampling frame and who had a primary or secondary diagnosis of NAS (ICD-9-CM code 779.5) were also included in the study $(n=2)$.

Additionally, the study utilized a theoretical framework and captured homogeneity among NAS-related hospital protocols. Relevant covariates were chosen via support of the literature and utilization of a biopsychosocial theoretical framework. Although utilization of 
EMR data for a retrospective chart review was necessary, it is limiting as this data was collected for clinical, not research, purposes. EMR data can include high missingness and issues related to heterogeneity of information stored across patients' records. Although the study was conducted at one major birthing hospital (verse a multi-site study), it allowed for homogeneity among trained staff on NAS-related maternal substance use and neonatal exposure screening methodology and NAS scoring/diagnosis and treatment protocols.

\section{Conclusions}

Although substance use occurrence and documentation of drug class-specific substances consumed during pregnancy is often unknown and/or difficult to detect, identification of exposure type can impact clinical applications. Identification of drug class-specific exposure can particularly impact NAS-related diagnoses, determined severity, and treatment protocols. The current study supports numerous drug class-specific exposures that can be linked to NAS and there are variations among these exposures with severity of NAS or NAS requiring treatment. Future research should focus on the application and effectiveness of substance specific neonatal exposure screening tools and tailored treatment protocols to most effectively target drug specific withdrawal. 


\title{
Chapter 4
}

Assessment of in utero opiate and cocaine exposure detection: A comparison of meconium analysis to alternative mother-infant biological and diagnostic indicators

\begin{abstract}
Objectives: Opioid and cocaine antenatal substance use can result in significant obstetric and pediatric health implications. Accurate detection of in utero exposed neonates can improve patient care and health outcomes. Therefore, we assessed the effectiveness of opiate and cocaine antenatal exposure among mother-infant biological and diagnostic indicators collected at labor and delivery hospital admission. Method: A retrospective medical chart review included 381 mother-infant dyads exposed to antenatal substances who had a meconium analysis conducted at delivery between January $1^{\text {st }} 2009$ and March $3^{\text {rd }} 2014$. Alternative biological and diagnostic indicators of maternal urine drug screens (UDS), maternal substance use ICD-9-CM codes, and neonatal exposure diagnostic ICD-9-CM codes were compared against meconium results. Performance of detection methods were analyzed via sensitivity and specificity analyses. Results: Meconium identified the highest incidence of antenatal cocaine (5\%) and opioid (41\%) in utero exposures. Among opiate exposures, maternal UDS had the highest sensitivity [.57, 95\% CI: .45, .69] and specificity [.90, 95\% CI: .83, .98] values. Cocaine had higher overall accuracy scores, with the highest sensitivity value of maternal substance use diagnosis [.37, 95\% CI: .15, .59] and the highest specificity value of maternal UDS [.99, 95\% CI: .98, 1.0]. Conclusion: Alternative detection measures collected at delivery were ineffective at identifying in utero substance exposure, especially neonatal exposed $I C D-9-C M$ codes. Findings indicate many exposed neonates could be misdiagnosed or left untreated. Accurate antenatal exposure identification at delivery is an important form of tertiary care that warrants development of improved detection methods with standardized biological identification protocols.
\end{abstract}




\subsection{Introduction}

Opioid and/or cocaine use during pregnancy can result in substantial negative healthrelated outcomes for the mother and newborn. Women who use substances during pregnancy are at an increased medical and obstetric risk for poor nutrition, anemia, problems related to infections, preterm labor, miscarriage, placental abruption, and postpartum hemorrhage.[142] Newborns with intrauterine exposure to opioids and cocaine are at an increased risk of prematurity, low birthweight, neurobehavioral symptoms, congenital malformations, neonatal abstinence syndrome, longer hospital stays, and are more likely to be placed in foster care or adoption.[11, 22, 143]

Despite the associated negative health implications, the occurrence of opioid and substance use during pregnancy is alarmingly high. Antenatal licit and illicit opiate use has increased nearly 5-fold in the United States between 2000 and 2009.[17] Although cocaine use during pregnancy has declined since the mid-1980's, it remains a significant public health threat, as cocaine is still the second most common illicit drug of abuse among pregnant women.[144]

Ideally, substance use among women of childbearing age could be eliminated at the primary prevention-level or at least substance using women could be identified early on in pregnancy at the secondary prevention-level. Benefits of early identification of antenatal opioid and/or cocaine use include educational parenting interventions, maternal drug treatment enrollment (such as methadone or buprenorphine maintained therapy), and increased medical observation during pregnancy.

Although the greatest harm reduction and cost-benefit approach would be prevention or early detection, it is not always possible to detect maternal opioid and cocaine use during pregnancy prior to labor and delivery (LD) hospital admission. Many substance using women 
have unplanned pregnancies and often lack adequate prenatal care.[145] In addition to logistic barriers, antenatal substance use is associated with a plethora of maternal biopsychosocial risk factors, such as poverty, psychiatric comorbidities, lack of social support, physical abuse, sexually transmitted infections, and poor prenatal nutrition. $[22,143]$

Many in utero substance exposed neonates go undetected, typically until LD hospital admission where neonatal and maternal biological drug screens are conducted and/or neonatal withdrawal symptoms are identified.[43, 44] Therefore, from a practical perspective it is important to assess the effectiveness of drug detection screening measures collected at delivery, where clinical data will be collected on every patient.

There is currently no optimal screening tool for identifying antenatal substance use, though reviews outline pros and cons of various non-invasive screening methods utilized at LD admission.[45, 115, 146] Open-ended and non-judgmental structured maternal interviewing can be effective, especially for identifying cannabis use, but is susceptible to recall, interviewer, and response bias.[46, 69, 115] Maternal urine drug screening (UDS) has several clinical indications with minimal logistical barriers, though it is associated with a short window of detection and thus it has a low sensitivity. Maternal hair analysis can produce high sensitivity diagnostics, but it is associated with high false positive rates and collection issues, as long hair segments are needed to widen the gestational detection window.[46, 50]. Neonatal urine analysis is considered a traditional drug screening method, though it is limited by the faulty collection methods and a short detection window.[45] Meconium, or the first stool that the neonate passes, is the specimen of choice for in utero exposure detection because it exhibits high sensitivity, it is easy to collect, and it has a large window of detection (i.e., it begins to form weeks $12^{\text {th }}$ to $16^{\text {th }}$ gestation). [45, 48, 147, 148] Although meconium serves as a reliable reservoir for drug and metabolite 
compounds, drawbacks include the necessity of confirmation tests to avoid false-positives, the possibility of contaminated samples, and the comparatively slow turnaround time to obtain confirmed test results. A newer method of umbilical cord tissue specimen mimics the strengths of meconium drug screening, with additional pros of a faster turnaround of results and the guarantee that every neonate will have a testable specimen.[45] The limitation to cord tissue analysis is the lack of literature supporting interpretation of results and known disposition of drugs in the specimen.[116]

While taking the strengths and limitations of drug screening methods into account, increased medical observation of in utero substance exposure is necessary. Antenatal substance exposure can result in NAS, which requires early identification and treatment post-delivery. NAS is a constellation of central nervous system, metabolic vasomotor, respiratory, and gastrointestinal disturbances a newborn experiences while withdrawing from in utero substance exposure.[12] Neonatal abstinence syndrome primarily results from opioid in utero exposure though other drugs, such as cocaine, have been linked to neonatal withdrawal.[22] Signs and symptoms of neonatal withdrawal typically occur until 28 to 72 hours after birth and severe cases require pharmacological treatment.[11] Screening and detection is crucial because undiagnosed newborns could be released prematurely from the hospital, resulting in untreated withdrawal in a less stable environment.

Considering the public health threat of substance use during pregnancy and the benefits of accurate detection, alternative screening methods of maternal and neonatal diagnostic codes and biological drug tests were assessed in an at-risk patient population. The purpose of the current study was to assess the effectiveness of antenatal substance use detection among different mother-infant dyad identification methods conducted at LD hospital admission. 


\subsection{Methods}

\section{Sample}

The current study aimed to compare sensitivity and specificity of various screening tests to identify cocaine and opioid in utero exposed infants. A subpopulation was utilized from a larger retrospective cohort of substance-using women who delivered a singleton live birth (International Classification of Disease, Ninth Revision, Clinical Modification (ICD-9-CM) code V27.0) at a major birthing center in north-central WV between January 1, 2009 and March 3, 2014. The original sample was restricted to mother-infants dyads of women aged 18 years or older at delivery and newborns of at least 28 weeks gestational age. If a mother gave birth to multiple babies during the study time frame, the first born that fit the sample criteria was included in the study.

Inclusion criteria of the larger retrospective cohort consisted of women who had an active drug use diagnosis at delivery (ICD-9-CM: 303.9x, 304.x, 305.x, 648.31, 648.32, 649.01, 649.02), a positive urine drug screen (for any drug) during the nine months prior to delivery admission, were prescribed prescriptions of buprenorphine or methadone at delivery admission prior to giving birth [61], and/or who delivered a baby diagnosed with NAS (ICD-9-CM: 779.5). Mother-infant dyads were excluded if the mother did not deliver at the study hospital $(n=5)$ or if the neonate expired at the same delivery admission prior to collection of a meconium sample $(n=7)$. After the 12 exclusions, the original sample included 560 mother-infant dyads. Study diagnostic indictors where derived from $I C D-9-C M$ codes because $I C D-10-C M$ codes were not utilized during the study period. Please see the section 3.2 of this dissertation for additional detailed information on the sample criteria and data collection methodology. 
Out of a possible 560 dyads, the current study utilized the subpopulation of 381 motherinfant dyads that had a neonatal meconium drug screen on record. Most $(n=118)$ of the 179 infants that did not have a meconium test result were born after a universal neonatal drug screen policy change that took place at the study hospital. Universal umbilical cord tissue drug screens were initiated on all neonates born at the study hospital from March $1^{\text {st }} 2013$ onward. Prior to universal cord tissue testing, meconium drug screens were conducted on all newborns suspected of substance exposure. The study sample came from an at-risk patient population, therefore those born prior to universal cord tissue testing without a meconium result on record $(n=61)$ most likely resulted from unavailable and/or compromised specimen samples for confirmation testing or, although less likely, they could have been missed via risk-based screening.

\section{Determination of Cocaine and Opioid Use in Meconium Samples}

As per hospital protocol, at least four grams of neonatal meconium specimens were collected for routine clinical purposes and sent for analysis to Associated Regional and University Pathologists (ARUP) laboratories in Salt Lake City, Utah. Specimens were stored and transported at 20-25 degrees Celsius. Results were reported within one to four days of the lab receiving the specimen. All valid meconium samples were screened by enzyme-linked immunosorbent assay (ELISA) to identify the appropriate drug class. A limitation of meconium drug screening is its ability to produce false positive results. Therefore, positive results were followed by a confirmation test of a fully validated gas chromatography-mass spectrometry or liquid chromatography-tandem mass spectrometry. Cocaine was screened as its own drug class. For the purpose of this study, opioid exposure was indicated if the neonate had a positive meconium result in the drug screen panel categories of opiates, methadone, and/or propoxyphene. Please see Table 4.1 for the size of the sample required for testing, the screening 
cutoff (or concentration at which the test can detect a drug or metabolite), and the confirmation cutoff for positive screens.

\section{Maternal Urine Drug Screen}

Maternal UDS were taken at the corresponding LD hospital admission. Tests were ordered in the obstetrical setting by the maternal infant care center department as part of a universal or risk-based screening. Universal maternal UDS was initiated on January 1, 2011 at the study hospital. Prior to universal testing, women who had a documented, suspected, or acknowledge maternal history of drug use (of which are included in the study's cohort) or had insufficient prenatal care were screened.

Enzyme Immunoassay methodology was utilized with a minimum collection volume of one milliliter of urine at the study hospital's laboratory, which was the performing laboratory. Specimens were stored at ambient temperatures up to 24 hours and could be refrigerated up to 5 days. Confirmation of positive results were confirmed by mass spectrometry techniques.

For the purpose of the current study, only information on cocaine and opioid test results were collected. Cocaine remained consistent on the UDS panel as 'cocaine metabolite' with the decision cutoff level of $300 \mathrm{ng} / \mathrm{mL}$. Therefore, all mothers with a documented UDS at LD were included in the cocaine UDS-specific analysis $(n=327)$. During the study period the UDS panels for opioid detection slightly evolved. Between May and June 2011, the opioid UDS panel changed to include buprenorphine as a separate component with a cutoff level at $10 \mathrm{ng} / \mathrm{mL}$. In order to be properly compared to the meconium drug screen (without a buprenorphine panel), we only compared the maternal opioid UDS panels to meconium $(n=128)$.

Maternal UDS tests were determined to be positive for opioids if methadone, opiate, and/or propoxyphene substances were detected at the $300 \mathrm{ng} / \mathrm{mL}$ level. Out of the 381 mother- 
infant dyads, 199 mothers had the new UDS at LD admission (with buprenorphine), 128 had the old UDS without buprenorphine, and 54 mothers were not given a UDS at delivery or their specimen collection was not documented in the patients' electronic medical records.

\section{Maternal ICD-9-CM Diagnosis}

Diagnostic criteria of maternal substance use for opioids and/or cocaine were collected at LD hospital admission. Opioid use during pregnancy was captured if the electronic medical records had an ICD-9-CM diagnostic code of 304.00, 304.01, 304.70, 304.71, 305.50, and/or 304.51. Cocaine use was captured if the electronic medical records had an $I C D-9-C M$ diagnostic code of 304.20, 304.21, 305.60, and/or 305.61. These diagnoses were defined as current use (i.e., not in remission) as a single or polysubstance exposure and ranged from dependence to nondependent opioid and cocaine abuse.

\section{Neonatal ICD-9-CM Diagnosis}

Neonatal in utero substance exposure was measured via exposure diagnostic codes in the neonates' electronic medical record at first hospital admission (i.e., birth). Diagnosis of cocaine and opioid exposure was determined from ICD-9-CM codes 760.72 and 760.75 (i.e., noxious influences affecting the fetus and/or neonate).

\section{Maternal and Neonatal Characteristics}

In order to better describe the study patient population, maternal sociodemographic and obstetric-related characteristics were captured. Easily accessible clinical parameters were collected from patient's electronic medical records via manual researcher and automatic hospital decision support analyst extraction (see section 3.2).

Maternal characteristics captured at delivery included age, years of education completed, marital status, Medicaid insurance type, residence, and history of substance use and/or mental 
health treatment. Marital status was operationalized as married, single, or other, with 'other' indicating mothers who were divorced, legally separated, or widowed. Residence was categorized as rural, urban, and metropolitan and it was determined from patient's county of residence Rural-Urban 2013 Continuum Codes (RUCC).[76] Other covariates included selective serotonin reuptake inhibitors (SSRIs) taken during pregnancy, maternal smoking status, nulliparous and gravidity status, spontaneous abortions, number of prenatal care visits, and mode of delivery (i.e., vaginal or cesarean). Nulliparous status and maternal gravidity did not include the current birth (i.e., mother-infant dyad). Spontaneous abortions were defined as a nontherapeutic miscarriage before 20 weeks gestational age. Neonatal variables included gestational age (weeks) and other growth parameters comprising of head circumference (centimeters), length (centimeters), and weight (grams) measured at delivery.

\section{Statistical Analysis}

Descriptive statistics were utilized to better explain patient characteristics. Categorical variables were represented as frequencies and percentages, normally distributed continuous variables were displayed as mean and standard deviations, and skewed continuous variables were presented as median and interquartile ranges. Patient characteristics were stratified by a positive cocaine and/or opioid meconium test and deemed significant via Chi-square test and student's ttest bivariate analysis.

Maternal UDS results, substance use diagnoses, and infant exposure diagnoses were examined as alternative in utero substance exposure screening methods. These methods were compared against meconium drug screens as the defined 'gold standard'. Performance of the screening tools were assessed by sensitivity, specificity, positive predictive value, negative predictive value, and accuracy measures (see Appendix F).[149] 
Sensitivity was defined as the number of true positives divided by the number of all positive assessments (i.e., meconium tests). Specificity was the number of true negative assessments divided by the number of all negative assessments. Positive predictive values (PPV) equaled the number of true positives divided by the number of screening positives and negative predictive values (NPV) was the number of true negatives divided by the number of screening negatives. Accuracy was defined as the number of correct assessments divided by the number of all assessments. True positive was defined as mother-infant dyads with a positive meconium result and, for the same drug of interest, a positive alternative screen (i.e., maternal UDS, maternal $I C D-9-C M$ diagnostic use, and neonatal $I C D-9-C M$ exposure). Alternatively, true negative was defined as mother-infants dyads who had a negative meconium result and had a negative alternative drug screen.

Binomial proportion estimates and asymptotic standard error (ASE) 95\% confidence intervals were reported. If two proportions had non-overlapping confidence intervals then they were considered significantly different.[150] All analyses were conducted using SAS 9.4 and significance was defined with a 2-tailed alpha of .05 (SAS Institute Inc., Cary, North Carolina). Ethical approval of the current study was obtained from the Institutional Review Board of the author's academic institution.

\subsection{Results}

\section{Characteristics of Patients}

Of 381 mother-infant dyads with a corresponding meconium drug screen, $165(43.3 \%)$ were positive for cocaine or opioids (see Table 4.2). At delivery admission, mothers on average were 26 years of age, had 12 years of completed education, a gravidity of 2.8 , had 0 spontaneous abortions, and had 9 prenatal care visits. Most women were single, had Medicaid insurance, lived 
in a metropolitan defined area, had a history of drug/mental health treatment, smoked cigarettes at LD hospital admission, already delivered another child at time of current birth, and had a vaginal mode of delivery for the birth under study. Although on the lower end of head width and weight, newborn growth parameters were within typical ranges with a patient average gestational age of 37.6 weeks, head width of 32.8 centimeters, length of 47.6 centimeters, and weight of 2,812.3 grams.[151]

Mothers who delivered a newborn with a positive meconium drug screen for cocaine or opioids were significantly less likely to be first time moms and had more previous pregnancies (gravidity). Newborns with a positive meconium drug screen were significantly younger (gestational age), shorter (length), and weighed less at delivery compared to newborns without a positive cocaine or opioid meconium test.

\section{Screening Test Performance}

In utero exposure of maternal cocaine and opioid use was observed via neonatal meconium drug screens, maternal UDS, maternal diagnostic substance use codes, and neonatal diagnostic exposure codes (see Table 4.3). Meconium analysis detected the highest incidence of antenatal cocaine use (5\%) and opioid use (40.9\%).

Detection of in utero cocaine and opioid exposure was then compared between the three screening tests and meconium drug screens as the gold standard. For opioid exposure, maternal UDS had the highest sensitivity (56.7\%) and specificity value (90.2\%). Maternal UDS had the highest PPV (86.4\%) and maternal ICD-9-CM diagnostic code had the highest NPV (67.5\%). Maternal UDS had the highest accuracy value of $65.4 \%$.

For the detection of cocaine, maternal ICD-9-CM diagnosis had the highest sensitivity value (37\%) and maternal UDS had the highest specificity value (99.7\%). Maternal UDS also 
had the highest PPV (85.7\%), while maternal ICD-9-CM diagnosis had the highest NPV (96.8\%). Maternal UDS had the overall highest accuracy value of $96.3 \%$.

\subsection{Discussion}

\section{Screening Test Performance}

The current study assessed the performance of alternative screening methods taken at labor and delivery that detect whether a neonate was exposed to cocaine and/or opioids in utero. Maternal UDS, maternal ICD-9-CM substance use diagnostic codes, and neonatal ICD-9-CM exposure codes were compared to neonatal meconium drug screen results. Overall, there was surprisingly low sensitivity and moderate to high specificity. This indicates if a neonate's meconium result for opioids or cocaine was negative then the alternative screening measure would most likely be negative. In addition to specificity, negative predictive values (NPV) were higher than positive predictive values (PPV). Therefore, among those who had a negative screen, the probability of having a negative meconium test result was greater than the probability of having a positive meconium result among those with a positive screen. Neonatal $I C D-9-C M$ exposure codes had the poorest performance, while maternal ICD-9-CM and UDS screens were fairly similar across measures and drug-specific detection. A previous study that assessed highrisk maternal-infant dyads for in utero opiate and cocaine exposure similarly calculated higher diagnostic values for specificity than sensitivity.[46] However, their sensitivity measures to detect in utero substance exposure via structured maternal interview, maternal hair analysis, and meconium drug analysis were considerably higher than those of the current study. This is likely due to the different screening measures and techniques utilized. Women were screened throughout pregnancy and the researchers compared one result with the result of the other two methods, with both serving as the referent (i.e., if one of the two references were positive then 
the referent was positive). The current study is the first to compare meconium results to alternative screening methods of maternal and/or neonatal $I C D-9-C M$ codes for substance use detection performance.

Screening performance varied between methods within drug class exposures. Within opioid tests, maternal UDS had a significantly higher sensitivity score compared to neonatal ICD-9-CM. Maternal UDS also had a significantly higher NPV score compared to neonatal ICD9- $C M$ exposure codes and maternal substance use $I C D-9-C M$ codes. Within cocaine tests, there were no significant differences among accuracy tests between alternative screening methods. Previous research found meconium analysis estimates of drug use during pregnancy were more sensitive than maternal interviews for both opiate and cocaine use, though maternal UDS was not assessed.[68] In addition, Ostre at al. found performance variation of in utero substance detection between screening tools (i.e., maternal interview, maternal hair analysis, and meconium analysis) among both cocaine and opiate exposure groups. Maternal hair analysis had the highest sensitivity, with meconium being the second highest due to the high false-positive hair analysis results.[46]

Comparing between opioid and cocaine in utero exposure, cocaine specificity tests were significantly higher across all measures (maternal UDS, maternal ICD-9-CM, and neonatal ICD9-CM) compared to opioid positive results. Cocaine NPV scores had a significantly higher NPV across all alternative screening measures compared to opioid tests. In addition, cocaine had a higher accuracy score across all screening methods compared to opioid tests. Although cocaine had low surprisingly low sensitivity values, overall alternative screening methods more accurately detected cocaine in utero exposure compared to opioid exposure. Although Ostre et al. 
utilized different screening tools, the findings were in agreement that overall better detection estimates were obtained with cocaine compared to opioids.[46]

\section{Characteristics of Patients}

The current study consists of substance exposed cohort, with $43 \%$ of the neonates specifically positive for cocaine and opioids. The remaining $57 \%$ could be positive for drugs

other than cocaine and/or opioids or they could have had an exposure level that did not result in a positive meconium drug screen. That is, their metabolites could be lower than the meconium analysis cut-off values. With respect to the percent of in utero cocaine and opioid exposure captured via the four screening tools, meconium detected the highest number of positive cocaine and opioid results and second highest number of positive opioid results. Maternal UDS captured the highest number of positive opioid results.

The majority of mothers in the study population had Medicaid insurance, smoked at LD admission, and had a history of drug/mental health treatment, and on average completed 12 years of education. Most women were single mothers and had at least one previous pregnancy. Women attended an average of 9 prenatal care visits, which is lower than the recommended number of 15 visits for low to normal risk pregnancies. The frequency of prenatal care visits throughout pregnancy should increase for high-risk patients, which is indicative of the study population because of the substance-using pregnant cohort and high occurrence of cesarean deliveries (i.e., $37 \%) \cdot[152,153]$

Although the study's overall number of prenatal visits was low, the number of visits were even lower among cocaine and/or opioid positive neonates (i.e., an average of 7.9). Substance abuse treatment integrated into prenatal care visits is linked with improved maternal and neonatal health.[154, 155] Therefore, this finding highlights a public health opportunity for treatment of 
maternal drug use during pregnancy. Specifically, women in the current study were at the hospital on average 9 times at which early detection and intervention could take place.

Positive meconium cocaine and/or opioid results were significantly associated with increased number of previous pregnancies and poor neonatal growth parameters (i.e., younger gestational age, shorter length, and lower weight), compared to non-positive cocaine/opioid results. This finding is in agreement with existing literature and highlights the importance of tertiary care to prevent substance use during future pregnancies.[46, 68]

\section{Clinical Implications}

Study findings indicate that overall, the alternative screening methods had a moderate to high specificity and low sensitivity estimates compared to meconium drug tests.[156] Thus, if a neonate had a positive cocaine or opiate meconium result it is likely that the alternative screening methods would not detect antenatal substance exposure. Accurate detection of in utero exposed neonates is extremely important. This form of tertiary prevention (i.e., detection at delivery) could result in increased length of medical observation during the newborn period, in order to properly diagnose and treat the newborn for potential withdrawal. Additional benefits include short and long term pediatric follow-up and, if necessary, breastfeeding restrictions. Indirect benefits to identifying antenatal substance use at LD hospital admission include prevention of postnatal infant exposure and decreased risk of antenatal drug use in future pregnancies.[115] This finding is important since mothers in the present study who gave birth to a newborn with a positive meconium result for cocaine or opiates were at an increased likelihood to have had multiple pregnancies. If exposure at LD can be identified, treatments and interventions can not only help the newborn infant but also future children of these mothers. 
Overall, despite the low sensitivity measures, alternative mother-infant biological and diagnostic indicators performed better for cocaine than opioid in utero exposure. Recent trends of substance use during pregnancy among women delivering in WV indicate that antenatal cocaine use is decreasing, while opiate use has greatly increased (see chapter 2). Therefore, development of more accurate opiate in utero substance exposure is warranted.

Neonatal ICD-9-CM diagnostic exposure codes performed poorly as detection for in utero substance exposure, which indicated the study hospital is not properly utilizing this form of screening. These codes should perfectly match the meconium results, as exposure diagnoses are based off of mother opioid maintenance therapy enrollment, maternal self-report of use, or maternal and/or neonatal biologic drug screens. This finding is of clinical importance because utilization of these diagnostic codes are vital for research purposes, as they are widely used to quantify the magnitude of this public health problem.[157] Poor data collection may result in faulty research regarding epidemic detection, hypothesis generation, and evaluation of public health intervention, policy and clinical practice. Furthermore, inaccurate data could lead to inappropriate allocation of public health prevention and resources (e.g., fiscal or treatment support).

\section{Limitations}

While interpreting the study findings, readers should be aware of potential limitations. Neonatal meconium drug tests were utilized as the gold standard, to which other screening methods were compared. Although meconium screening is favored for its long window of detection and ease of collection, potential drawbacks include availability of uncompromised specimens for every neonate and comparatively slow testing turnaround time.[69] With these 
pros and cons in mind, it is important to note that mass meconium drug screening in a clinical setting is feasible and affordable, with costs approximating urine toxicology analysis.[46, 47]

Another limitation includes the sparse use of antenatal cocaine among the study population. The few number of mother-infant dyads with meconium samples that tested positive for cocaine likely resulted in the surprisingly low sensitivity and high specificity of cocainespecific results. Also, the current study was conducted at a single hospital and therefore screening diagnostic generalizability of maternal substance use and neonatal exposure ICD-9$C M$ codes could be lacking. However, comparisons between meconium and maternal urine drug screens are likely generalizable because specimens were collected and sent to a national reference laboratory for results. All positive results are confirmed in order to reduce falsepositives. Although this step is in place, it is important to consider the study findings with a focus on medical detection, evaluation, and treatment, rather than in the context of criminal prosecution.[146]

\section{Conclusion}

Alternative screening methods to detect in utero cocaine and opiate exposure at LD hospital admission are specific but not sensitive in comparison to meconium test results. Therefore, if a neonate has a negative meconium drug screen then they will likely have a negative maternal UDS result, maternal substance use diagnosis, and/or neonatal exposure diagnosis screening. In addition, a positive meconium test result will not likely be detected via alternative screening methods at LD admission and therefore many exposed neonates would go undetected. Neonatal ICD-9-CM exposure codes have particularly low sensitivity values, indicating a lack of correct identification of exposed neonates via internationally recognized ICD-9-CM diagnostic codes. 


\section{Chapter 5}

\section{Discussion}

\subsection{Summary of Key Findings}

This project utilized WV Health Care Authority Uniform Billing data between 2007 and 2013 to describe sub-state regional incidence rates and trends of neonatal antenatal exposure and NAS diagnoses. Data from electronic medical records were also obtained to identify risk of NAS and NAS requiring pharmacologic treatment among substance-using pregnant women who gave birth at Ruby Memorial Hospital between 2009 and March $3^{\text {rd }} 2014$. A subpopulation of this cohort was utilized to assess the effectiveness of maternal urine drug screens, maternal substance use $I C D-9-C M$ diagnostic codes, and neonatal $I C D-9-C M$ exposure codes in detecting antenatal substance use. The research concluded an overall increasing trend of NAS diagnoses in WV with sub-state variations, which are substantially higher than national averages. The research also established variation among drug class-specific in utero exposures and risk of NAS as well as low sensitivity measures of alternative in utero exposure detection methods taken at labor and delivery from a single hospital site.

West Virginia statewide estimates of NAS rates, trend over time, and documented neonatal exposure was not previously unknown. The current study addressed this gap in the literature and calculated that the statewide NAS incidence rate increased over 4-fold from 7.74 to 31.56 per 1,000 live births per year between 2007 and 2013. Study findings, compared to available national estimates from 2009 to 2012, indicated that the WV rates increased more than three times that of national annual averages.[16, 17]

Between 2007 and 2013, all but one of the six sub-state regions significantly increased (region 3 did not significantly change). In 2013, the southeastern region of the state had the 
highest NAS rate with an IR of 48.76 per 1,000 live births. During the study period, neonatal opioid exposure significantly increased, cocaine exposure signficantly decreased, and the rate of hallucinogen exposure remained unchanged. Exposure rates were substantially lower than NAS diagnostic rates. In addition, NAS infants were more likely to experience clinical conditions (i.e., respiratory diagnoses, feeding difficulty, and seizures), longer hospital length of stay, and were more likely to be born to a mother insured by Medicaid.

A retrospective medical chart review of mother-infant dyads with antenatal substance exposure was conducted at a major WV birthing hospital. This data was obtained in order to fill the gap in the literature regarding risk of NAS among non-opioid in utero substance exposed neonates. Drug class-specific exposure was defined via neonatal meconium drug screen results. The substance exposed cohort had a 29\% risk of being diagnosed with NAS and among infants diagnosed 54\% had NAS that required pharmacological treatment. Risk of NAS was highest among opioid only and poly-substance with opioid exposures. This finding held consistent in the regression model controlling for potential confounders. That is, neonates with opioid only or poly-substance with opioid in utero exposures were significantly more likely to be diagnosed with NAS compared to neonates with a non-positive meconium drug screen. Number of prenatal care visits and maternal opioid maintenance therapy enrollment were independent risk factors for delivering a newborn diagnosed with NAS. The drug class-specific exposure group rankings varied between risk of NAS and NAS requiring pharmacological treatment. Among neonates diagnosed with NAS, sedative only exposure and poly-substance with opioids had the highest and second highest risk of NAS requiring treatment, respectively.

A subpopulation $(\mathrm{n}=381)$ of the substance exposed cohort from a retrospective medical chart review was utilized to assess the effectiveness of in utero opioid and cocaine exposure 
diagnostic indicators collected at labor and delivery. This was the first study to evaluate the effectiveness of in utero exposure detection among maternal and neonatal $I C D-9-C M$ diagnostic codes compared to a neonatal biological drug screen. Neonates with a positive drug screen $(n=165$ or $43.3 \%)$ had a significantly younger gestational age, shorter birthlength, and lighter birthweight compared to newborns without a positive screen. In addition, women who delivered a newborn with a positive meconium screen were more likely to have a higher previous gravidity and were less likely to be first time mothers.

Meconium analysis detected the highest incidence of antenatal cocaine and opiate in utero exposure and was deemed the gold standard. Therefore, the alternative identification methods of maternal urine drug screens (UDS), maternal ICD-9-CM substance use diagnoses, and neonatal exposure $I C D-9-C M$ diagnoses were compared to meconium results. Overall, the alternative methods exhibited surprisingly low sensitivity and moderate to high specificity measures. Neonatal ICD-9-CM exposure codes had the poorest overall performance, while maternal UDS had the highest accuracy estimate. Despite low sensitivity measures, overall the alternative detection methods more accurately identified cocaine in utero exposure compared to opioid exposure.

Screening performance varied among alternative methods, within and between antenatal opioid and cocaine exposures. Within opioid drug testing, Maternal UDS had significantly higher sensitivity scores compared to neonatal $I C D-9-C M$ and significantly higher NPV scores compared to neonatal exposure and maternal substance use ICD-9-CM codes. There were no significant differences among screening performance within cocaine drug testing. Screening performance between antenatal exposure types showed cocaine specificity tests were significantly higher across all alternative measures compared to opioid positive results. In 
addition, cocaine NPV scores were significantly higher across all alternative screening methods compared to opioid detection.

\subsection{Significance of the Study}

The upward trend of NAS throughout WV is indicative of a rise in an at-risk pregnant patient population in which NAS diagnoses are occurring at a much higher rate than what is observed at the national level. NAS rates were the highest among patients who reside in the southeastern sub-state region. Consequently, throughout the study period only one federally funded opioid maintenance therapy facility that accepted pregnant women was located in this region. $[85,100]$ There is a unique need for antenatal public health drug treatment and preventative interventions in $\mathrm{WV}$, specifcially targeted to the southeastern region of the state. These findings suggest health policy officials should allocate resources to the southeast region in order to improve access to substance use prevention efforts, treatment, and health care planning during pregnancy.

In addition, the WV and sub-state NAS trends highlighted an increase in opiate and decrease in cocaine in utero exposure via $I C D-9-C M$ neonatal diagnostic codes. This finding is clinically relevant because it could indicate a potential shift in the type of maternal antenatal substance use. For clarification, this 'shift' would be described as a change in prevalence at the population-level, as opposed to individual women switching from cocaine to opiate use during pregnancy. Knowledge of population trends in type of drug use within a specific group can aid in tailored public health prevention and outreach efforts.

Neonatal substance exposure rates were far lower than NAS rates. This finding indicates underutilization of appropriate neonatal ICD-9-CM codes among WV birthing hospitals. Therefore, utilization of this diagnostic codes for research and quality improvement purposes 
could greatly underestimate the targeted vulnerable population. Improved reporting of neonatal exposure-specific diagnoses could advance effective research and surveillance efforts.

The second study indicated that multiple substances could lead to a NAS diagnosis and that variations existed between drug class-specific exposure and heightened risk of NAS/NAS requiring treatment. Although the majority of NAS cases were linked to an opioid antenatal exposure, other substances can cross the placenta barrier and lead to an infant's expression and consequently a diagnosis of NAS.[11,22] The study also indicated independent maternal risk factors associated with delivering an infant diagnosed with NAS. This information could improve health care professionals' ability to identify high risk mothers, in order to indicate earlier substance use screening and ensure extended mother-infant observation. The clinical significance lies in the fact that withdrawal signs and symptoms can start 48 to 72 hours after birth, therefore undiagnosed infants could be sent home prior to the onset of withdrawal.[11, 42]

Knowledge of risk of NAS and NAS requiring treatment among non-opioid in utero exposed infants is important because, regardless of the exposure, NAS infants are largely being diagnosed and treated via the same hospital protocols. The most common scoring tool utilized for NAS diagnosis, treatment, dosage, and discharge guidelines was created specifically for opiate withdrawal in term infants.[143] This can be problematic because although numerous drug exposures can result in similar withdrawal signs and symptoms, there are likely some differences that could result in a misdiagnosed case of NAS.[22] In addition, traditional treatment guidelines recommend an opioid-based first line of treatment (i.e., morphine or methadone).[11] Although opioid therapy for NAS management helps with bowl motility inhibition and treatment of seizures, it might not be ideal for non-opioid induced NAS as iatrogenic (or hospital-induced) NAS is a rising concern.[13, 158] 
The third study examined the effectiveness of in utero opiate and cocaine detection among alternative mother-infant biological and diagnostic indicators taken at labor and delivery hospital admission. Overall, alternative methods of maternal urine drug screen, maternal substance use diagnosis, and neonatal exposure diagnosis performed poorly with low sensitivity values compared to meconium analysis results. Therefore, solely utilizing these alternative screening methods taken at labor and delivery would likely underestimate the number of exposed newborns. This finding is of clinical importance because the use of ineffective detection methods could result in unidentified newborns that would likely be left untreated and/or released from the hospital prior to withdrawal symptom onset.[42] Identifying effective detection of antenatal substance use at delivery is a form of tertiary prevention that could increase the length of medical observation during the newborn period for diagnostics and treatment purposes. In addition, accurate detection can help ensure pediatric follow-up, set potential breastfeeding restrictions, prevent other postnatal exposures, and decrease risk of substance use during future pregnancies.

Neonatal ICD-9-CM diagnostic exposure codes had the lowest performance measures, further indicating a lack of neonatal exposure code utilization at a major WV birthing hospital. These diagnostic indicators are important to ensure exposure-specific tailored treatment protocols for NAS. In addition, these codes are widely used amongst researchers to quantify the occurrence and trend of NAS and to evaluate related public health interventions, policy, and clinical practices.[157] Accurate use of neonatal exposure indicators is also important in order to appropriately allocate public health prevention and treatment resources to combat NAS and substance use during pregnancy. 


\subsection{Strengths and limitations}

There are inherent issues associated with WV Health Care Authority Uniform Billing data. Reporting behavior could be revenue driven, thus if the costliness of a NAS diagnosis changed over time then an influx in NAS cases could be a result from reporting behavioral differences verses actual change in disease occurrence. In addition, population-based surveillance is dependent on accurate statewide standardized coding practices. Potential hospital billing practices and NAS diagnosis definitions could have been variable during the assessment period and change over time. Increased medical awareness of the syndrome and more accurate diagnoses from birthing hospitals located close to major medical centers could also potentially inflate NAS rates. Alternatively, hospital billing data could led to underreported NAS and neonatal substance exposure diagnoses if not all newborns were drug screened and/or if exposure status was based on maternal self-report. Although inaccurate rates due to the above mentioned reasons are possible, it is unlikely because NAS rates paralleled the opioid epidemic, drug overdose death rates, and rise in painkiller prescribing rates.

West Virginia identifiable data were unavailable, therefore hospital discharges, rather than individual patients, were the unit of observation. Although unlikely, due to the window of withdrawal and natural course of the syndrome, NAS discharge data could result in duplication if the same patient (i.e., a newborn) had multiple hospital admissions related to the diagnosis. Duplication would likely underestimated NAS rates because it would increase the total population (i.e., denominator) while the NAS cases (numerator) would remain constant as this condition is likely to be diagnosed at the first hospital admission. These potential problems are driven from the study design and data source utilized in the first study, thus solutions are limited and constraints may simply be a limitation of the data. The chosen data source is beneficial 
because it is currently the best approach to obtaining retrospective statewide NAS rates of WV residents as it is the only data source that currently captures all insurance types. Strictly using Medicaid data could overestimate NAS rates because it would mostly capture the at-risk population.

Potential limitations of the second and third study associated with the data source include reliance of medical records, vulnerability to misinformation, and potential selection bias. There are inherent issues relying on medical record data; such that, missing and/nor inaccurate information might exist. Misinformation would most likely occur with identified maternal characteristics and inaccurate exposure classification. If a patient is asked about their medical history or substance use behavior before or during pregnancy data could be susceptible to recall or reporting bias, especially if the mother feels judged or pressured to answer a certain way.[46] Misclassification in neonatal in utero exposure classification could occur if they were exposed to substances not included in the biological analysis drug panel and/or if the exposure was not capture via the drug screen due to concentrations below a screening cutoff value. Selection bias could occur by determining which patients met the study eligibility criteria. These potential issues were combated with manual review of every maternal-infant electronic record for accuracy and detailed data extraction. Extraction of maternal and neonatal characteristics were chosen based off of supportive literature and the utilization of the biopsychosocial theoretical framework. In addition, a broad inclusion criteria of substance use during pregnancy was utilized that helped ensure a complete cohort of in utero exposed neonates. The study population was therefore more generalizable to a substance using pregnant population, which allowed more appropriate examination of multiple drug class antenatal exposures. 
The second and third study utilized data collected at a single hospital. Although this could be viewed as a limitation with respect to generalizability, it resulted as a study strength because NAS protocols were established and well defined throughout the study period. This standardization better enabled the researchers to assume homogeneity within the data. The hospital staff had a standardized neonatal exposure screening methodology and was uniformly trained on NAS scoring/diagnostic and treatment protocols.

There are pros and cons associated with utilizing umbilical cord tissue and meconium specimen for drug screening (please see chapters 1 and 3 for more details). Although strengths and weakness of the non-invasive biological drug tests can be argued, they are considered the gold standard for neonatal in utero substance exposure detection.[45] The study hospital had a protocol in place to universally screen newborns and mothers (via urine drug screens), which has supported findings of feasible and affordable mass meconium and umbilical cord tissue drug screening in clinical settings. [46, 47]

\subsection{Future Research}

West Virginia and sub-state NAS rates are sustainably higher than the national average and are continuing to trend upwards. The relationship between sub-state NAS rates, the proportion of available treatment options for pregnant women, and region-level maternal and societal characteristics is not yet known. Future research should examine geographic availability of opioid treatment programs for pregnant women and associated likelihood of delivering a newborn with a NAS diagnosis, NAS requiring pharmacological treatment, and other neonatal clinical outcomes. Potential barriers to treatment accessibility for this vulnerable population should be identified, in order to improve patient care. 
The first study found an increase in opioid and decrease in cocaine neonatal exposure diagnoses over time. Future research should target a potential population shift in maternal drug use, as findings could indicate need based treatment options for substance using pregnant women. In addition, future research should target the lack of neonatal exposure $I C D-9-C M$ code utilization. Policy changes related to mandating the assignment of neonatal exposure diagnostic codes with every NAS diagnosis or adding NAS to WV's list of reportable disease is worth exploring. Future research should explore the potential benefits of implementing an interoperable insurance/hospital system and/or statewide standardized surveillance system to track NAS diagnoses and neonatal exposure codes.

Results for the second study indicate non-opioid antenatal exposures are linked to NAS. This finding raises clinical concerns because currently practiced NAS diagnostic and treatment protocols are based on opioid in utero exposures. Future research should focus on the effectiveness of current standardized diagnostic and treatment opioid-based hospital protocols, in instances when there is a known non-opioid exposure status. Creation of an exposure-tailored diagnosis, assessment, and treatment protocol might be warranted.

The second study also found variation among the hierarchical ordering of exposures associated with risk between NAS diagnosis and severity of NAS. Specifically, opioid exposure (single or multiple substances) had the highest risk of NAS diagnoses, but sedative only in utero exposure had the highest risk of NAS requiring treatment. Future research should further explore this association. If sedative only in utero exposure leads to more severe NAS, compared to opiate exposures, than reconsideration of the typical first-line treatment is warranted. Specifically, future research should explore the effectiveness of phenobarbital or clonidine as a first-line 
treatment for sedative-exposed neonates, as opposed to the typical first-line opioid based medication.

With respect to the third study, future research should target development of more accurate in utero substance exposure screening methods. Despite the low sensitivity detection methods for cocaine exposure, overall cocaine detection out performed opiate exposure detection. This finding coupled with the increased use of opioids during pregnancy highlight the importance of accurate detection methods, specifically for opiate in utero exposure. In addition, the third study only assessed in utero opiate and cocaine exposure among mother-infant biological and diagnostic indicators, taken at labor and delivery, compared to meconium analysis. Therefore, future studies should assess alternative drug class exposures and screening measures via a multi-site study.

In conclusion, study findings warrant future research that aim to identify the potential harm of utilizing opioid-based hospital screening, diagnostic, and treatment protocols for neonates with non-opioid in utero exposure. Tailored antenatal exposure-specific hospital protocols might improve neonatal withdrawal symptoms and shorten hospital length of stay, as well as prevent the possibility of iatrogenic NAS. The study also warrants a statewide surveillance system to track NAS and neonatal exposure diagnoses, in order to estimate the magnitude of the health problem, detect epidemics, and assess the effectiveness of health practice or new policy measures. The study highlights a need for greater prevention and treatment resources in $\mathrm{WV}$, as well as accurate/feasible non-invasive exposure detection methods. Early detection of substance use during pregnancy, coupled with integrated and comprehensive multidisciplinary services, could improve treatment compliancy and maternal and neonatal health outcomes. In addition, pregnancy can serve as an opportune window for public 
health intervention because women are motivated to get and stay clean. Health care providers should focus on facilitating a caring relationship with their patients via non-judgmental behaviors, in order to develop a strong support network, provide additional parenting skills, educate parents on NAS, and prevent a future delivery of a NAS diagnosed infant.[159-161] A multi-faceted approach should also be taken to address NAS from a primary prevention standpoint. The public health goal is to deter opioid misuse during the preconception period. Emerical evidence suggests patient-provider discussions regarding appropriate use of contraceptive devices and the potential health risks of opiate use during pregnancy can help prevent fetal substance expsoure and thus minimize the emerging public health threat of NAS.[94, 162] Prescribing clinician's can also use of the state prescription drug monitoring program and required counsel about risk of opiate use during pregnancy to women of childbearing age whom are prescribed narcotics.[94] 


\section{References}

1. Substance Abuse and Mental Health Services Administration, Results from the 2013 National Survey on Drug Use and Health: Summary of National Findings in HHS Publication No. (SMA) 14-4863, N.S. H-48, Editor. 2014: Rockville, MD.

2. Substance Abuse and Mental Health Services Administration, Results from the 2010 National Survey on Drug Use and Health: Summary of National Findings in HHS Publication No. (SMA) 11-4658, N.S. H-41, 2011: Rockville, MD.

3. Substance Abuse and Mental Health Services Administration., Trends in Substance of Abuse among Pregnant Women and Women of Childbearing Age in Treatment in The TEDS Report. 2013.

4. Stitely, M.L., et al., Prevalence of drug use in pregnant West Virginia patients. The West Virginia Medical Journal, 2010. 106(4 Spec No): p. 48-52.

5. Paulozzi, L.J., K.A. Mack, and J.M. Hockenberry, Vital signs: variation among States in prescribing of opioid pain relievers and benzodiazepines - United States, 2012. MMWR Morb Mortal Wkly Rep, 2014. 63(26): p. 563-8.

6. National Institute on Drug Abuse, Prenatal Exposure to Drugs of Abuse: A Research Update from the National Institute on Drug Abuse, in Topics in Brief. 2011.

7. Pacifici, G.M. and R. Nottoli, Placental transfer of drugs administered to the mother. Clin Pharmacokinet, 1995. 28(3): p. 235-69.

8. Niebyl, J. and J. Simpson, Tertology and drugs in pregnancy, G.1.w.s. med., Editor. 2008.

9. Wilson, J.G., Environment and Birth Defects. 1973, New York: Academic Press. 
10. Brown, H.L. Stages of Development of the Fetus. Women's Health Issues 2015; Available from: http://www.merckmanuals.com/home/women-s-health-issues/normalpregnancy/stages-of-development-of-the-fetus.

11. Hudak, M.L. and R.C. Tan, Neonatal drug withdrawal. Pediatrics, 2012. 129(2): p. e540e560.

12. The American Congress of Obstetricians and Gynecologists, ACOG Committee Opinion No. 524: Opioid abuse, dependence, and addiction in pregnancy. Obstetrics and Gynecology, 2012. 119(5): p. 1070-1076.

13. Lewis, T., et al., Pharmacoepidemiology of opiate use in the neonatal ICU: Increasing cumulative doses and iatrogenic opiate withdrawal. J Opioid Manag, 2015. 11(4): p. 30512.

14. Cramton, R.E. and N.E. Gruchala, Babies breaking bad: neonatal and iatrogenic withdrawal syndromes. Curr Opin Pediatr, 2013. 25(4): p. 532-42.

15. Jansson, L.M., M. Velez, and C. Harrow, The opioid-exposed newborn: assessment and pharmacologic management. J Opioid Manag, 2009. 5(1): p. 47-55.

16. Patrick, S.W., et al., Increasing incidence and geographic distribution of neonatal abstinence syndrome: United States 2009 to 2012. J Perinatol, 2015.

17. Patrick, S.W., et al., Neonatal Abstinence Syndrome and Associated Health Care Expenditures United States, 2000-2009. JAMA: Journal of the American Medical Association, 2012. 307(18): p. 1934-1940.

18. Knoppert, D., The worldwide opioid epidemic: implications for treatment and research in pregnancy and the newborn. Paediatric Drugs, 2011. 13(5): p. 277-279. 
19. Paulozzi, L., et al., Vital signs: overdoses of prescription opioid pain relievers---United States, 1999--2008. MMWR Morb Mortal Wkly Rep, 2011. 60(43): p. 1487-92.

20. Bateman, B.T., et al., Patterns of opioid utilization in pregnancy in a large cohort of commercial insurance beneficiaries in the United States. Anesthesiology, 2014. 120(5): p. 1216-24.

21. Desai, R.J., et al., Increase in Prescription Opioid Use During Pregnancy Among Medicaid-Enrolled Women. Obstetrics \& Gynecology, 2014. 123(5): p. 997-1002.

22. Jansson, L.M. and M.L. Velez, Infants of drug-dependent mothers. Pediatr Rev, 2011. 32(1): p. 5-12; quiz 12-3.

23. Pierog, S., O. Chandavasu, and I. Wexler, Withdrawal symptoms in infants with the fetal alcohol syndrome. The Journal Of Pediatrics, 1977. 90(4): p. 630-633.

24. Robe, L.B., D.S. Gromisch, and S. Iosub, Symptoms of neonatal ethanol withdrawal. Curr Alcohol, 1981. 8: p. 485-93.

25. Coles, C.D., et al., Neonatal ethanol withdrawal: characteristics in clinically normal, nondysmorphic neonates. J Pediatr, 1984. 105(3): p. 445-51.

26. Godding, V., et al., Does in utero exposure to heavy maternal smoking induce nicotine withdrawal symptoms in neonates? Pediatr Res, 2004. 55(4): p. 645-51.

27. Pichini, S. and O. Garcia-Algar, In utero exposure to smoking and newborn neurobehavior: how to assess neonatal withdrawal syndrome? Therapeutic Drug Monitoring, 2006. 28(3): p. 288-290.

28. Law, K.L., et al., Smoking during pregnancy and newborn neurobehavior. Pediatrics, 2003. 111(6 Pt 1): p. 1318-23. 
29. Choo, R.E., et al., Neonatal abstinence syndrome in methadone-exposed infants is altered by level of prenatal tobacco exposure. Drug \& Alcohol Dependence, 2004. 75(3): p. 253260.

30. Lester, B.M. and J.F. Padbury, Third pathophysiology of prenatal cocaine exposure. Developmental Neuroscience, 2009. 31(1-2): p. 23-35.

31. Coles, C.D., et al., Effects of cocaine and alcohol use in pregnancy on neonatal growth and neurobehavioral status. Neurotoxicol Teratol, 1992. 14(1): p. 23-33.

32. Bauer, C.R., et al., Acute neonatal effects of cocaine exposure during pregnancy. Archives Of Pediatrics \& Adolescent Medicine, 2005. 159(9): p. 824-834.

33. Wachsman, L., et al., What happens to babies exposed to phencyclidine (PCP) in utero? Am J Drug Alcohol Abuse, 1989. 15(1): p. 31-9.

34. Smith, L., et al., Effects of prenatal methamphetamine exposure on fetal growth and drug withdrawal symptoms in infants born at term. J Dev Behav Pediatr, 2003. 24(1): p. 17-23.

35. Rementeria, J.L. and K. Bhatt, Withdrawal symptoms in neonates from intrauterine exposure to diazepam. J Pediatr, 1977. 90(1): p. 123-6.

36. Athinarayanan, P., et al., Chloriazepoxide withdrawal in the neonate. Am J Obstet Gynecol, 1976. 124(2): p. 212-3.

37. Sanz, E.J., et al., Selective serotonin reuptake inhibitors in pregnant women and neonatal withdrawal syndrome: a database analysis. Lancet, 2005. 365(9458): p. 482-7.

38. Moore, S.J., et al., A clinical study of 57 children with fetal anticonvulsant syndromes. $\mathrm{J}$ Med Genet, 2000. 37(7): p. 489-97. 
39. Wachman, E.M., et al., The relationship between maternal opioid agonists and psychiatric medications on length of hospitalization for neonatal abstinence syndrome. $\mathrm{J}$ Addict Med, 2011. 5(4): p. 293-9.

40. Desmond, M.M., et al., Maternal barbiturate utilization and neonatal withdrawal symptomatology. J Pediatr, 1972. 80(2): p. 190-7.

41. Finnegan, L.P., Current therapy in neonatal perinatal medicine-2. In N. Nelson (Ed.), Neonatal Abstinence Syndrome . 1990, Toronto: BC Decker.

42. Wexelblatt, S.L., et al., Universal Maternal Drug Testing in a High-Prevalence Region of Prescription Opiate Abuse. Journal of Pediatrics, 2015. 166(3): p. 582-586.

43. Kelly, R., D. Zatzick, and T. Anders, The detection and treatment of psychiatric disorders and substance use among pregnant women cared for in obstetrics. Am J Psychiatry, 2001. 158(2): p. 213-9.

44. Carpenter, L., Nature of the problem and state of the field., in Presentation at the National AIA Resource Cetner Substance Exposed Newborns Conference. 2010: Alexandria, VA.

45. Gray, T. and M. Huestis, Bioanalytical procedures for monitoring in utero drug exposure. Analytical and bioanalytical chemistry, 2007. 388(7): p. 1455-1465.

46. Ostrea Jr, E.M., et al., Estimates of illicit drug use during pregnancy by maternal interview, hair analysis, and meconium analysis. The Journal of Pediatrics, 2001. 138(3): p. 344-348.

47. Ostrea, E.M., Jr., A. Romero, and H. Yee, Adaptation of the meconium drug test for mass screening. J Pediatr, 1993. 122(1): p. 152-4. 
48. Associated Regional and University Pathologists (ARUP), Newborn Drug ScreeningMeconium and Umbilical Cord Testing. The Physician's Guide to Laboratory Test Selection and Interpretation 2006; Available from:

\section{https://www.arupconsult.com/Topics/MeconiumandUmbilicalCord.html\#tabs=0.}

49. Montgomery, D., et al., Testing for fetal exposure to illicit drugs using umbilical cord tissue vs meconium. Journal of Perinatology, 2006. 26(1): p. 11-14.

50. Concheiro, M., et al., Alternative matrices for cocaine, heroin, and methadone in utero drug exposure detection. Ther Drug Monit, 2013. 35(4): p. 502-9.

51. Wilder, C.M. and T. Winhusen, Pharmacological Management of Opioid Use Disorder in Pregnant Women. CNS Drugs, 2015. 29(8): p. 625-36.

52. Jones, H.E., et al., Treatment of Opioid Dependent Pregnant Women: Clinical and Research Issues. Journal of substance abuse treatment, 2008. 35(3): p. 245-259.

53. McLemore, G.L., et al., Novel pharmacotherapeutic strategies for treatment of opioidinduced neonatal abstinence syndrome. Semin Fetal Neonatal Med, 2013. 18(1): p. 3541.

54. Neonatal drug withdrawal. American Academy of Pediatrics Committee on Drugs. Pediatrics, 1998. 101(6): p. 1079-88.

55. Casper, T. and M.W. Arbour, Identification of the pregnant woman who is using drugs: implications for perinatal and neonatal care. J Midwifery Womens Health, 2013. 58(6): p. 697-701.

56. Seligman, N.S., et al., Relationship between maternal methadone dose at delivery and neonatal abstinence syndrome. Journal of Pediatrics, 2010. 157(3): p. 428. 
57. Pritham, U.A., J.A. Paul, and M.J. Hayes, Opioid dependency in pregnancy and length of stay for neonatal abstinence syndrome. J Obstet Gynecol Neonatal Nurs, 2012. 41(2): p. $180-90$.

58. Patel, P., et al., Perinatal outcomes of Australian buprenorphine-exposed mothers and their newborn infants. J Paediatr Child Health, 2013. 49(9): p. 746-53.

59. Smirk, C.L., et al., Home-based detoxification for neonatal abstinence syndrome reduces length of hospital admission without prolonging treatment. Acta Paediatr, 2014. 103(6): p. 601-4.

60. Jansson, L.M. and M. Velez, Neonatal abstinence syndrome. Curr Opin Pediatr, 2012. 24(2): p. 252-8.

61. Desai, R.J., et al., Exposure to prescription opioid analgesics in utero and risk of neonatal abstinence syndrome: population based cohort study. BMJ, 2015. 350: p. h2102.

62. McQueen, K.A., J. Murphy-Oikonen, and L. Desaulniers, Maternal substance use and neonatal abstinence syndrome: A descriptive study. Maternal and Child Health Journal, 2015.

63. Cleary, B.J., et al., Methadone and perinatal outcomes: a prospective cohort study. Addiction, 2012. 107(8): p. 1482-92.

64. Hanna, E.Z., V.B. Faden, and M.C. Dufour, The effects of substance use during gestation on birth outcome, infant and maternal health. J Subst Abuse, 1997. 9: p. 111-25.

65. Lund, I.O., et al., Prescription drug use among pregnant women in opioid Maintenance Treatment. Addiction, 2013. 108(2): p. 367-76. 
66. McGlone, L., et al., Visual outcome in infants born to drug-misusing mothers prescribed methadone in pregnancy. Br J Ophthalmol, 2014. 98(2): p. 238-45.

67. Varner, M.W., et al., Association between stillbirth and illicit drug use and smoking during pregnancy. Obstet Gynecol, 2014. 123(1): p. 113-25.

68. Pichini, S., et al., Assessment of exposure to opiates and cocaine during pregnancy in a Mediterranean city: preliminary results of the "Meconium Project". Forensic Sci Int, 2005. 153(1): p. 59-65.

69. Gray, T.R., et al., Identification of prenatal amphetamines exposure by maternal interview and meconium toxicology in the Infant Development, Environment and Lifestyle (IDEAL) study. Ther Drug Monit, 2009. 31(6): p. 769-75.

70. Engel, G., The need for a new medical model: a challenge for biomedicine. Science, 1977. 196(4286): p. 129-136.

71. Substance Abuse and Mental Helath Services Administration, The NSDUH Report: Substance Use Disorders in Substate Regions: 2008 to 2010. 2012: Center for Behavioral Health Statistics and Quality: Rockville, MD.

72. Stokes, M., C. Davis, and G. Koch, Categorical Data Analysis Using SAS®, Third Edition. 2012, Cary, NC: SAS Institute Inc.

73. Greenland, S., Modeling and variable selection in epidemiologic analysis. Am J Public Health, 1989. 79(3): p. 340-9.

74. Manchikanti, L., et al., Opioid epidemic in the United States. Pain Physician, 2012. 15(3 Suppl): p. Es9-38. 
75. Trust for America's Health, Key Health Data About West Virginia. 2015; Available from: http://www.healthyamericans.org/states/?stateid=WV\#section=4,year=2014, code=hrsamc h.

76. United States Census Bureau, 2010 Census Urban and Rural Classification and Urban Area Criteria. Geographic Concepts 2015 February 09, 2015; Available from: https://www.census.gov/geo/reference/ua/uafaq.html.

77. Appalachian Regional Commission, The Appalachian Region. 2008; Available from: http://www.arc.gov/appalachian_region/mapofappalachia.asp.

78. Levi, J., L. Segal, and A. Martin, The facts hurt: A state-by-state injury prevention policy report, Trust for America's Health, Editor. 2015: Washington, DC.

79. Office of Rural Health Policy, List of rural counties and designated eligible census tracts in metropolitan counties: Updated census 2010, U.S.D.o.H.a.H. Services, Editor. 2013: Rockville, MD.

80. Burns, L., et al., Geographic and maternal characteristics associated with alcohol use in pregnancy. Alcohol Clin Exp Res, 2011. 35(7): p. 1230-7.

81. Shannon, L.M., J.R. Havens, and L. Hays, Examining differences in substance use among rural and urban pregnant women. Am J Addict, 2010. 19(6): p. 467-73.

82. Jumah, N.A., L. Graves, and M. Kahan, The management of opioid dependence during pregnancy in rural and remote settings. Cmaj, 2015. 187(1): p. E41-6.

83. Richardson, R., S. Bolisetty, and C. Ingall, The profile of substance-using pregnant mothers and their newborns at a regional rural hospital in New South Wales. The Australian \& New Zealand Journal Of Obstetrics \& Gynaecology, 2001. 41(4): p. 415419. 
84. Bailey, B.A. and L.K. Cole, Rurality and birth outcomes: findings from southern appalachia and the potential role of pregnancy smoking. J Rural Health, 2009. 25(2): p. 141-9.

85. Lander, L.R., et al., Rural healthcare disparities: challenges and solutions for the pregnant opioid-dependent population. W V Med J, 2013. 109(4): p. 22-7.

86. Hart, L.G., et al., Rural health care providers in the United States. J Rural Health, 2002. 18 Suppl: p. 211-32.

87. Lisonkova, S., et al., Birth outcomes among older mothers in rural versus urban areas: a residence-based approach. J Rural Health, 2011. 27(2): p. 211-9.

88. West Virginia Vital Statistics, 2010, West Virginia Bureau for Public Health, Health Statistics Center, Editor. 2013.

89. Jones, H.E., et al., Neonatal abstinence syndrome after methadone or buprenorphine exposure. N Engl J Med, 2010. 363(24): p. 2320-31.

90. Adams M, A.G., Kirby R, Wingate M, Perinatal Epidemiology for Public Health Practice. 2009, New York, NY: Springer Science+Buisness Media, LLC.

91. ArcGIS Desktop: Release 10.2. 2013: Redlands, CA: Environmental Systems Research Institute.

92. Livingston, S., Neonates Exposed to Opioids in Vermont: Vermont Uniform Hospital Discharge Data Set, V.D.o. Health, Editor. 2014.

93. Massatti, R., Falb, M., Yors, A., Potts, L., Beeghly, C. \& Starr, S., Neonatal abstinence syndrome and drug use among pregnant women in Ohio, 2004-2011., O.D.o.M.H.a.A. Services, Editor. 2013: Columbus, OH. 
94. Association of State and Territorial Health Officials (ASTHO), Neonatal abstinence syndrome: How states can help advance the knowledge base for primary prevention and best practices of care. 2014: Arlington, VA.

95. National Institue on Drug Abuse (NIDA). Overdose Death Rates, National Institue of Health. 2015; Available from: http://www.drugabuse.gov/related-topics/trends-

\section{statistics/overdose-death-rates.}

96. Purdue Healthcare Advisors, 2012 West Virginia State Health Profile: Shaping safe and healthy communities, Department of Health and Human Resources. WV Bureau for Public Health, Editor. 2012.

97. Rossen, L.M., D. Khan, and M. Warner, Trends and geographic patterns in drugpoisoning death rates in the U.S., 1999-2009. Am J Prev Med, 2013. 45(6): p. e19-25.

98. West Virginia Division of Juvenile Services, West Virginia Incident-Based Reporting System (WVIBRS), Department of Military Affairs and Public Safety: Division of Justice and Community Services, Editor. 2015: Charleston, West Virginia.

99. Area Health Resources Files (AHRF), US Department of Health and Human Services, Health Resources and Services Administration, Bureau of Health Workforce, Editor. 2013-2014: Rockville, MD.

100. Substance Abuse and Mental Health Services Administration, Opioid Treatment Program Directory, in Medication-Assisted Treatment for Substance Use Disorders, Department of Health and Human Services, Center for Substance Abuse Treatment, Division of Pharmacologic Therapies, Editor.: Rockville, MD.

101. Meyer, M., et al., Development of a substance abuse program for opioid-dependent nonurban pregnant women improves outcome. J Addict Med, 2012. 6(2): p. 124-30. 
102. Beardsley, K., et al., Distance traveled to outpatient drug treatment and client retention. J Subst Abuse Treat, 2003. 25(4): p. 279-85.

103. Office of National Drug Control Policy, Appalachia HIDTA. High-Intensity Drug Trafficking Areas 1998; Available from: https://www.ncjrs.gov/ondcppubs/publications/enforce/hidta2001/appl-fs.html.

104. Executive Office of the President, High Intensity Drug Trafficking Areas Program Report to Congress, Office of National Drug Control Policy, Editor. 2011.

105. Drug Enforcement Administration. West Virginia DEA Resources. Available from: http://www.drugenforcementedu.org/west-virginia/.

106. Warren, M.D., et al., Implementation of a statewide surveillance system for neonatal abstinence syndrome - tennessee, 2013. MMWR Morb Mortal Wkly Rep, 2015. 64(5): p. $125-8$.

107. Kocherlakota, P., Neonatal abstinence syndrome. Pediatrics, 2014. 134(2): p. e547-61.

108. O'Donnell, M., et al., Increasing prevalence of neonatal withdrawal syndrome: population study of maternal factors and child protection involvement. Pediatrics, 2009. 123(4): p. e614-21.

109. Burns, L. and R.P. Mattick, Using population data to examine the prevalence and correlates of neonatal abstinence syndrome. Drug Alcohol Rev, 2007. 26(5): p. 487-92.

110. Zuckerman, B., Drug-Exposed Infants: Understanding the Medical Risk. The Future of Children, 1991. 1(1): p. 26-35.

111. Salisbury, A.L., et al., Fetal effects of psychoactive drugs. Clinics in Perinatology, 2009. 36(3): p. 595-619. 
112. Doberczak, T.M., et al., Impact of maternal drug dependency on birth weight and head circumference of offspring. Am J Dis Child, 1987. 141(11): p. 1163-7.

113. Wachman, E.M., et al., Association of OPRM1 and COMT single-nucleotide polymorphisms with hospital length of stay and treatment of neonatal abstinence syndrome. JAMA, 2013. 309(17): p. 1821-7.

114. Bar-Oz, B., et al., Comparison of meconium and neonatal hair analysis for detection of gestational exposure to drugs of abuse. Arch Dis Child Fetal Neonatal Ed, 2003. 88(2): p. F98-F100.

115. Kwong, T.C. and R.M. Ryan, Detection of intrauterine illicit drug exposure by newborn drug testing. National Academy of Clinical Biochemistry. Clin Chem, 1997. 43(1): p. $235-42$.

116. de Castro, A., et al., Methadone, cocaine, opiates, and metabolite disposition in umbilical cord and correlations to maternal methadone dose and neonatal outcomes. Ther Drug Monit, 2011. 33(4): p. 443-52.

117. Choo, R.E., et al., Determination of methadone, 2-ethylidene-1,5-dimethyl-3,3diphenylpyrrolidine, 2-ethyl-5-methyl-3,3-diphenylpyraline and methadol in meconium by liquid chromatography atmospheric pressure chemical ionization tandem mass spectrometry. J Chromatogr B Analyt Technol Biomed Life Sci, 2005. 814(2): p. 369-73.

118. de Castro, A., et al., Development and validation of a liquid chromatography mass spectrometry assay for the simultaneous quantification of methadone, cocaine, opiates and metabolites in human umbilical cord. J Chromatogr B Analyt Technol Biomed Life Sci, 2009. 877(27): p. 3065-71. 
119. Marin, S.J., et al., Detection of neonatal drug exposure using umbilical cord tissue and liquid chromatography time-of-flight mass spectrometry. Ther Drug Monit, 2014. 36(1): p. 119-24.

120. Montgomery, D.P., et al., Using umbilical cord tissue to detect fetal exposure to illicit drugs: a multicentered study in Utah and New Jersey. J Perinatol, 2008. 28(11): p. 750-3.

121. Lind, J.N., et al., Infant and maternal characteristics in neonatal abstinence syndrome-selected hospitals in Florida, 2010-2011. MMWR Morb Mortal Wkly Rep, 2015. 64(8): p. 213-6.

122. Greig, E., A. Ash, and A. Douiri, Maternal and neonatal outcomes following methadone substitution during pregnancy. Archives Of Gynecology And Obstetrics, 2012. 286(4): p. 843-851.

123. Taylor, L., et al., Clinical features and correlates of outcomes for high-risk, marginalized mothers and newborn infants engaged with a specialist perinatal and family drug health service. Obstet Gynecol Int, 2012. 2012: p. 867265.

124. Haller, D.L., et al., Perinatal substance abusers. Psychological and social characteristics. J Nerv Ment Dis, 1993. 181(8): p. 509-13.

125. Creanga, A.A., et al., Maternal drug use and its effect on neonates: a population-based study in Washington State, in Obstet Gynecol. 2012: United States. p. 924-33.

126. Fingar KR, S.C., Weiss AJ, Owens PL, Neonatal and Maternal Hospital Stays Related to Substance Use, 2006-2012, in HCUP Statistical Brief \#193. 2015, Agency for Healthcare Research and Quality: Rockville, MD.

127. United States Census Bureau, Metropolitan and Micropolitan. February 26, 2013; Available from: http://www.census.gov/population/metro/about/. 
128. Ness, R.B., et al., Cocaine and tobacco use and the risk of spontaneous abortion. N Engl J Med, 1999. 340(5): p. 333-9.

129. Jackson, A. and L. Shannon, Factors Associated With the Chronicity of Intimate Partner Violence Experiences Among Pregnant Women in Detoxification Services. Women Health, 2015: p. 1-17.

130. Kaltenbach, K., et al., Predicting treatment for neonatal abstinence syndrome in infants born to women maintained on opioid agonist medication. Addiction, 2012. 107 Suppl 1: p. $45-52$.

131. Kandall, S.R., et al., The methadone-maintained pregnancy. Clin Perinatol, 1999. 26(1): p. 173-83.

132. Jones, H.E., et al., Neonatal abstinence syndrome after methadone or buprenorphine exposure. New England Journal of Medicine, 2010. 363(24): p. 2320-2331.

133. Kellogg, A., et al., Current trends in narcotic use in pregnancy and neonatal outcomes. American Journal Of Obstetrics And Gynecology, 2011. 204(3): p. 259.e1-4.

134. Fulroth, R., B. Phillips, and D.J. Durand, Perinatal outcome of infants exposed to cocaine and/or heroin in utero. Am J Dis Child, 1989. 143(8): p. 905-10.

135. Levinson-Castiel, R., et al., Neonatal abstinence syndrome after in utero exposure to selective serotonin reuptake inhibitors in term infants. Arch Pediatr Adolesc Med, 2006. 160(2): p. 173-6.

136. Sharpe, C. and C. Kuschel, Outcomes of infants born to mothers receiving methadone for pain management in pregnancy. Arch Dis Child Fetal Neonatal Ed, 2004. 89(1): p. F336. 
137. Alroomi, L.G., et al., Maternal narcotic abuse and the newborn. Archives of Disease in Childhood, 1988. 63(1): p. 81-83.

138. Sarkar, S. and S.M. Donn, Management of neonatal abstinence syndrome in neonatal intensive care units: a national survey. Journal of Perinatology, 2006. 26(1): p. 15-17.

139. Bagley, S.M., et al., Review of the assessment and management of neonatal abstinence syndrome. Addiction Science \& Clinical Practice, 2014. 9(1): p. 19-19.

140. Kandall, S.R. and L.M. Gartner, Late presentation of drug withdrawal symptoms in newborns. Am J Dis Child, 1974. 127(1): p. 58-61.

141. Kieviet, N., K.M. Dolman, and A. Honig, The use of psychotropic medication during pregnancy: how about the newborn? Neuropsychiatr Dis Treat, 2013. 9: p. 1257-66.

142. Pinto, S.M., et al., Substance abuse during pregnancy: effect on pregnancy outcomes. Eur J Obstet Gynecol Reprod Biol, 2010. 150(2): p. 137-41.

143. Ogunyemi, D. and G.E. Hernandez-Loera, The impact of antenatal cocaine use on maternal characteristics and neonatal outcomes. J Matern Fetal Neonatal Med, 2004. 15(4): p. 253-9.

144. Pan, I.J. and H.-y. Yi, Prevalence of Hospitalized Live Births Affected by Alcohol and Drugs and Parturient Women Diagnosed with Substance Abuse at Liveborn Delivery: United States, 1999-2008. Maternal and child health journal, 2013. 17(4): p. 667-676.

145. Pagnini, D.L. and N.E. Reichman, Psychosocial factors and the timing of prenatal care among women in New Jersey's HealthStart program. Fam Plann Perspect, 2000. 32(2): p. $56-64$. 
146. Wong, S., A. Ordean, and M. Kahan, Substance use in pregnancy. Journal Of Obstetrics And Gynaecology Canada: JOGC = Journal D'obstétrique Et Gynécologie Du Canada: JOGC, 2011. 33(4): p. 367-384.

147. Gareri, J., J. Klein, and G. Koren, Drugs of abuse testing in meconium. Clin Chim Acta, 2006. 366(1-2): p. 101-11.

148. Lisowska-Myjak, B., [Meconium as a new biological material for detecting intrauterine exposure to toxic substances]. Ginekol Pol, 2005. 76(7): p. 571-9.

149. Zhu, W., N. Zeng, and N. Wang. Sensitivity, specificity, accuracy, associated confidence interval and roc analysis with practical SAS® implementations in NESUG Proceedings: Health Care and Life Sciences. 2010. Baltimore, Maryland.

150. Knezevic, A. Overlapping confidence intervals and statistical significance. 2008. Cornell University, Cornell Statistical Consulting Unit.

151. World Health Organization. WHO Child Growth Standards. 2015; Available from: http://www.who.int/childgrowth/en/.

152. Kogan, M.D., et al., Comparing mothers' reports on the content of prenatal care received with recommended national guidelines for care. Public Health Rep, 1994. 109(5): p. 63746.

153. Martin, J.A., et al., Births: Final Data for 2013, in National Vital Statistics Reports 2015.

154. Goler, N.C., et al., Substance abuse treatment linked with prenatal visits improves perinatal outcomes: a new standard. J Perinatol, 2008. 28(9): p. 597-603.

155. Armstrong, M.A., et al., Perinatal substance abuse intervention in obstetric clinics decreases adverse neonatal outcomes. J Perinatol, 2003. 23(1): p. 3-9. 
156. Identifying infants and young children with developmental disorders in the medical home: an algorithm for developmental surveillance and screening [corrected] [published erratum appears in PEDIATRICS 2006 Oct;118(4):1808-9]. Pediatrics, 2006. 118(1): p. 405-420.

157. Title V maternal and child health services block grant to states program: Guidance and forms for the Title V application/annual report, U.S. Department of Health and Human Services, Health Resources and Services Administration, Maternal and Child Health Bureau, Editor. 2015: Rockville, MD.

158. Bio, L.L., A. Siu, and C.Y. Poon, Update on the pharmacologic management of neonatal abstinence syndrome. J Perinatol, 2011. 31(11): p. 692-701.

159. Cleveland, L.M. and S.L. Gill, "Try not to judge": mothers of substance exposed infants. MCN Am J Matern Child Nurs, 2013. 38(4): p. 200-5.

160. Kramlich, D. and R. Kronk, Relational Care for Perinatal Substance Use: A Systematic Review. MCN Am J Matern Child Nurs, 2015. 40(5): p. 320-6.

161. Rizzo, R.A., et al., Parenting and concerns of pregnant women in buprenorphine treatment. MCN Am J Matern Child Nurs, 2014. 39(5): p. 319-24.

162. Chou, R., et al., Clinical guidelines for the use of chronic opioid therapy in chronic noncancer pain. J Pain, 2009. 10(2): p. 113-30. 


\section{Tables}

\section{Chapter 2 Table}

Table 2.1. West Virginia Delivery Characteristics by Neonatal Abstinence Syndrome: HCA,UB 2007-2013

\begin{tabular}{|c|c|c|c|c|}
\hline \multirow[b]{2}{*}{ Characteristics } & \multicolumn{3}{|c|}{ NAS } & \multirow[b]{2}{*}{ P-value } \\
\hline & Total & Yes & No & \\
\hline Total sample & 119605 & $1974(1.65 \%)$ & $117631(98.35 \%)$ & \\
\hline Male & $60923(50.94 \%)$ & $1053(53.34 \%)$ & $59870(50.90 \%)$ & $.0967^{\mathrm{a}}$ \\
\hline \multicolumn{5}{|l|}{ Neonatal substance exposure } \\
\hline Narcotics & & & & $<.0001^{\mathrm{a}}$ \\
\hline Yes & $543(0.45 \%)$ & $98(4.96 \%)$ & $445(0.38 \%)$ & \\
\hline No & $119062(99.55 \%)$ & $1957(99.14 \%)$ & $117526(99.91 \%)$ & \\
\hline \multicolumn{4}{|l|}{ Hallucinogenic agents ${ }^{\mathrm{d}}$} & $.0156^{\mathrm{b}}$ \\
\hline Yes & $32(0.03 \%)$ & -- & -- & \\
\hline No & $119573(99.97 \%)$ & $1957(99.14 \%)$ & $117526(99.91 \%)$ & \\
\hline Cocaine & & & & $<.0001^{\mathrm{b}}$ \\
\hline Yes & $122(0.10 \%)$ & $17(0.86 \%)$ & $105(0.09 \%)$ & \\
\hline No & $119483(99.90 \%)$ & $1957(99.14 \%)$ & $117526(99.91 \%)$ & \\
\hline \multicolumn{5}{|c|}{ Presence of neonatal conditions } \\
\hline Respiratory diagnoses & $10060(8.41 \%)$ & $400(20.26 \%)$ & $9660(8.21 \%)$ & $<.001^{\mathrm{a}}$ \\
\hline Feeding difficulty & $872(0.73 \%)$ & $51(2.58 \%)$ & $821(0.70 \%)$ & $<.001^{\mathrm{a}}$ \\
\hline Seizure & $158(0.13 \%)$ & $18(0.91 \%)$ & $140(0.12 \%)$ & $<.0001^{\mathrm{b}}$ \\
\hline \multicolumn{5}{|l|}{ Neonatal hospital length } \\
\hline $\begin{array}{l}\text { of stay, Median (IQR) } \\
\text { Insurance }\end{array}$ & $2(2,3)$ & $8(4,18)$ & $2(2,3)$ & $\begin{array}{l}<.001^{\mathrm{c}} \\
<001^{\mathrm{a}}\end{array}$ \\
\hline $\begin{array}{l}\text { Insurance } \\
\text { Medicaid }\end{array}$ & & & & $<.001^{\mathrm{a}}$ \\
\hline $\begin{array}{l}\text { Medicaid } \\
\text { Medicare }^{\mathrm{d}}\end{array}$ & $\begin{array}{l}65502(54.77 \%) \\
97(0.08 \%)\end{array}$ & $1680(85.11 \%)$ & $63822(54.26 \%)$ & \\
\hline $\begin{array}{l}\text { Medicare } \\
\text { Other }\end{array}$ & $45898(38.37 \%)$ & $277(14.03 \%)$ & $45621(38.78 \%)$ & \\
\hline PEIA & $8108(6.78 \%)$ & $16(0.81 \%)$ & $8092(6.88 \%)$ & \\
\hline
\end{tabular}

HCA,UB, Health Care Authority, Uniform Billing Database; NAS, Neonatal Abstinence Syndrome; TCHG, Total hospital charge; IQR, Interquartile range; PEIA, Public Employees Insurance Agency

\footnotetext{
${ }^{a}$ Pearson Chi-square

${ }^{\mathrm{b}}$ Two-sided Fisher's exact test

${ }^{c}$ Wilcoxon Ranked Sum

${ }^{\mathrm{d}}$ Variable is not stratified via NAS diagnosis in order to comply with the HCA UB data user agreement of not reporting data less than or equal to 10 counts per cell.
} 


\section{Chapter 3 Tables}

Table 3.1. Breakdown of neonatal biological drug screen results and drug-class exposure groups.*

\begin{tabular}{lllllll}
\hline Biological drug screen & Total & $\begin{array}{l}\text { Opioids } \\
\text { only }\end{array}$ & $\begin{array}{l}\text { Cannabis } \\
\text { only }\end{array}$ & $\begin{array}{l}\text { Poly- } \\
\text { substance } \\
\text { with opioids }\end{array}$ & $\begin{array}{l}\text { Other } \\
\text { positive }\end{array}$ \\
\hline Umbilical cord tissue $^{\mathrm{a}}$ & & & & & & \\
$\quad$ Opioids & 34 & 23 & 0 & 11 & 0 & \\
$\quad$ Stimulants & 4 & 0 & 0 & 2 & 2 & \\
$\quad$ Sedatives & 15 & 0 & 0 & 3 & 12 & \\
$\quad$ Cannabinoids & 15 & 0 & 7 & 8 & 1 & 52 \\
Total & 106 & 23 & 7 & 11 & 13 & \\
\hline Meconium $^{\mathrm{b}}$ & & & & & & \\
$\quad$ Opioids & 151 & 87 & 0 & 64 & 0 & \\
Stimulants & 23 & 0 & 0 & 12 & 11 & \\
Sedatives & 23 & 0 & 0 & 15 & 8 & \\
$\quad$ Cannabinoids & 138 & 0 & 87 & 46 & 5 & 166 \\
Total & 370 & 110 & 75 & 94 & 6 & \\
\hline
\end{tabular}

*The umbilical cord tissue and meconium drug screen test results are not mutually exclusive. The drug-class specific exposure categorization is mutually exclusive. There were no positive PCP results and therefore this drug was not included in the groupings.

${ }^{a}$ Umbilical cord tissue drug panel includes opioids: buprenorphine, codeine, dihyrocodenine, fentanyl, hydrocodone, hydromorphone, meperidine, methadone, EDDP, acetylmorphine, morphine, naloxone, naltrexone, oxycodone, oxymorphone, propoxyphene, tapentadol, tramadol, and desmehyltramadol, stimulants: amphetamine, benzoylecgonine, cocaethylene, cocaine, MDMA, MDA, methamphetamine, and phentermine, sedatives: alprazolam, butalbital, clonazepam, aminoclonazepam, flurazepam, desalkylflurazepam, ethylflurazepam, lorazepam, midazolam, nitrazepam, nordiazepam, oxazepam, phenobarbital, secobarbital, temazepam, triazolam, and zolpidem.

${ }^{\mathrm{b}}$ Meconium drug panel includes opioids: opiates, propoxyphene, and methadone and metabolite, stimulants: cocaine and amphetamines, and sedatives: barbituates and benzodiazepines. Both drug screens also include marijuana metabolite and phencyclidine (or PCP). 
Table 3.2. Risk of neonatal abstinence syndrome (NAS) and pharmacological treatment of NAS among a cohort of substance-using mother-infant dyads.

\begin{tabular}{|c|c|c|c|c|}
\hline & $\begin{array}{l}\text { NAS } \\
\text { cases/sample } \\
\text { size }\end{array}$ & Risk of NAS & $\begin{array}{l}\text { NAS requiring } \\
\text { treatment } \\
\text { cases/sample } \\
\text { size }\end{array}$ & $\begin{array}{l}\text { Risk of } \\
\text { NAS } \\
\text { requiring } \\
\text { treatment }\end{array}$ \\
\hline Total cohort & $139 / 476$ & $29.2 \%$ & $75 / 139$ & $54.0 \%$ \\
\hline \multicolumn{5}{|l|}{ Drug exposure } \\
\hline Opioids only & $50 / 110$ & $45.5 \%$ & $27 / 50$ & $54 \%$ \\
\hline Cannabis only & $12 / 94$ & $12.8 \%$ & $4 / 12$ & $33.3 \%$ \\
\hline Stimulants only & $1 / 9$ & $11.1 \%$ & $0 / 1$ & 0 \\
\hline Sedatives only & $5 / 15$ & $33.3 \%$ & $4 / 5$ & $80 \%$ \\
\hline $\begin{array}{l}\text { Poly-substance with } \\
\text { opioids }\end{array}$ & $31 / 75$ & $41.3 \%$ & $21 / 31$ & $67.7 \%$ \\
\hline $\begin{array}{l}\text { Poly-substance without } \\
\text { opioids }\end{array}$ & $2 / 7$ & $28.6 \%$ & $1 / 2$ & $50 \%$ \\
\hline Non-positive & $38 / 166$ & $22.9 \%$ & $18 / 38$ & $47.4 \%$ \\
\hline P-value* & $<.0001$ & & 0.2702 & \\
\hline
\end{tabular}

${ }^{*}$ Cochran-Mantel-Haenszel: General Association 
Table 3.3. Maternal and Neonatal Characteristics by neonatal abstinence syndrome diagnosis.

\begin{tabular}{|c|c|c|c|c|}
\hline Characteristics & Overall & NAS & No NAS & p-value \\
\hline Total & 476 & $139(29.2 \%)$ & $337(70.8 \%)$ & \\
\hline Year of delivery & & & & $0.51^{\mathrm{a}}$ \\
\hline 2009 & $61(12.8 \%)$ & $11(7.9 \%)$ & $50(14.8 \%)$ & \\
\hline 2010 & $75(15.8 \%)$ & $20(14.4 \%)$ & $55(16.3 \%)$ & \\
\hline 2011 & $87(18.3 \%)$ & $36(25.9 \%)$ & $51(15.1 \%)$ & \\
\hline 2012 & $105(22.1 \%)$ & $33(23.7 \%)$ & $72(21.4 \%)$ & \\
\hline 2013 & $131(27.5 \%)$ & $34(24.5 \%)$ & $97(28.8 \%)$ & \\
\hline 2014 & $17(3.6 \%)$ & $5(3.6 \%)$ & $12(3.6 \%)$ & \\
\hline Maternal age, mean (SD) & $26.1(5.1)$ & $26.3(4.4)$ & $26.0(5.3)$ & $0.61^{\mathrm{b}}$ \\
\hline $\begin{array}{l}\text { Years of maternal education, }{ }^{*} \\
\text { median (IQR) }\end{array}$ & $12(11,13)$ & $12(11,13)$ & $12(11,13)$ & $0.11^{\mathrm{c}}$ \\
\hline Marital status* & & & & $0.40^{\mathrm{d}}$ \\
\hline Married & $106(22.6 \%)$ & $27(19.7 \%)$ & $79(23.7 \%)$ & \\
\hline Single & $304(64.7 \%)$ & $95(69.3 \%)$ & $209(62.8 \%)$ & \\
\hline Other & $60(12.8 \%)$ & $15(11.0 \%)$ & $45(13.5 \%)$ & \\
\hline Medicaid insurance & $375(78.8 \%)$ & $110(79.1 \%)$ & $265(78.6 \%)$ & $0.90^{\mathrm{e}}$ \\
\hline Residence & & & & $0.64^{\mathrm{e}}$ \\
\hline Metropolitan & $256(54.6 \%)$ & $73(52.9 \%)$ & $183(55.3 \%)$ & \\
\hline Non-metropolitan & $213(45.4 \%)$ & $65(47.1 \%)$ & $148(44.7 \%)$ & \\
\hline Family visited at hospital & $374(91.7 \%)$ & $107(89.9 \%)$ & $267(92.4 \%)$ & $0.42^{\mathrm{e}}$ \\
\hline $\begin{array}{l}\text { Maternal diagnosis of } \\
\text { psychological condition }\end{array}$ & $100(21.0 \%)$ & $35(25.2 \%)$ & $65(19.3 \%)$ & $0.15^{\mathrm{e}}$ \\
\hline Physical abuse present & $8(1.7 \%)$ & $3(2.5 \%)$ & $6(1.8 \%)$ & $1.00^{\mathrm{f}}$ \\
\hline Reported familial support & $412(97.4 \%)$ & $115(96.6 \%)$ & $297(97.7 \%)$ & $0.52^{f}$ \\
\hline History of chronic pain* & $79(17.3 \%)$ & $30(22.9 \%)$ & $49(15.0 \%)$ & $0.04^{\mathrm{e}}$ \\
\hline $\begin{array}{l}\text { History of drug/mental health } \\
\text { treatment* }\end{array}$ & $268(57.0 \%)$ & $104(76.5 \%)$ & $164(49.1 \%)$ & $<.0001^{\mathrm{e}}$ \\
\hline Opioid maintenance therapy & $209(44.0 \%)$ & $104(74.8 \%)$ & $105(31.3 \%)$ & $<.0001^{\mathrm{e}}$ \\
\hline Smoking packs per day* & $0.5(0.2,1.0)$ & $0.5(0.2,1.0)$ & $0.5(0.2,1.0)$ & $0.11^{\mathrm{c}}$ \\
\hline SSRI use during pregnancy & $60(12.7 \%)$ & $14(10.1 \%)$ & $46(13.7 \%)$ & $0.29^{\mathrm{e}}$ \\
\hline Nulliparous & $177(37.2 \%)$ & $45(32.4 \%)$ & $132(39.2 \%)$ & $0.16^{\mathrm{e}}$ \\
\hline Gravidity, median (IQR) & $2.0(1,4)$ & $2(1,4)$ & $3(2,4)$ & $0.51^{\mathrm{c}}$ \\
\hline $\begin{array}{l}\text { Spontaneous abortions, } \\
\text { median (IQR) }\end{array}$ & $0(0,1)$ & $0(0,1)$ & $0(0,1)$ & $0.84^{\mathrm{c}}$ \\
\hline Prenatal visits, mean (SD) & $8.8(5.7)$ & $7.6(5.0)$ & $9.3(6.0)$ & $0.01^{\mathrm{b}}$ \\
\hline Delivery type & & $0.3362^{\mathrm{e}}$ & & \\
\hline Cesarean & $176(37.0 \%)$ & $56(40.3 \%)$ & $120(35.6 \%)$ & \\
\hline Vaginal & $300(63.0 \%)$ & $83(59.7 \%)$ & $217(64.4 \%)$ & \\
\hline $\begin{array}{l}\text { Maternal pre-pregnancy BMI, } \\
\text { median (IQR) }\end{array}$ & $22.6(19.7,27.5)$ & $22.3(19.7,27.0)$ & $22.7(19.7,27.5)$ & $0.47^{\mathrm{c}}$ \\
\hline Neonate sex & & & & $0.23^{\mathrm{e}}$ \\
\hline Male & $240(50.4 \%)$ & $76(54.7 \%)$ & $164(48.7 \%)$ & \\
\hline Female & $236(49.6 \%)$ & $63(45.3 \%)$ & $173(51.3 \%)$ & \\
\hline
\end{tabular}


Gestational age, median (IQR)

Head c (cm), median (IQR)

Length $(\mathrm{cm})$, mean $(\mathrm{SD})$

Weight (g), mean (SD)

Neonatal drug screen

specimen mode

Meconium

Cord tissue

Neonatal drug screen results**

Opioids only

Cannabis only

Poly-substance with

opioids

Other

Non-positive
$38.1(36.9,39.2) \quad 38.0(37,39)$

$33.0(31.5,34) \quad 33(31,34)$

$47.6(3.8)$

$2,816.7(620.8)$

$47.1(4.1)$

$2761.7(630.8)$

$368(77.3 \%)$

$108(22.7 \%)$

$110(23.1 \%)$

$94(19.8 \%)$

$75(15.8 \%)$

$31(6.5 \%)$

$166(34.9 \%)$

$33(23.7 \%)$

$50(36.0 \%)$

$12(8.6 \%)$

$31(22.3 \%)$
$38.3(36.7,39.3) \quad 0.25^{\mathrm{c}}$

$33(32,34) \quad 0.71^{\mathrm{c}}$

$47.8(3.7) \quad 0.10^{\mathrm{b}}$

$2839.4(616.1) \quad 0.22^{b}$

$262(77.7 \%)$

$75(22.3 \%)$

$0.72^{\mathrm{e}}$

$60(17.8 \%)$

$82(24.3 \%)$

$44(13.1 \%)$

$23(6.8 \%)$

$128(38.0 \%)$

NAS: Neonatal Abstinence Syndrome, STD: Standard deviation, IQR: Interquartile range, PPD:

Packs per day, SSRI: Selective serotonin re-uptake inhibitors, BMI: Body mass index, $\mathrm{cm}$ :

Centimeters, g: Grams

*Self-reported from patient

**Mutually exclusive in utero substance exposures via neonatal meconium or cord tissue drug screen test results.

$\%$ : Column percent (calculations of sample and percent do not include missing values).

${ }^{a}$ Cochran-Armitage Trend Test

${ }^{\mathrm{b}} \mathrm{T}$-Test

${ }^{c}$ Wilcoxon Ranked Sum

${ }^{\mathrm{d}}$ Cochran-Mantel-Haenszel: General Association

${ }^{\mathrm{e}}$ Pearson chi-square

${ }^{\mathrm{f}}$ Two-sided Fisher's exact test 
Table 3.4. Association between in utero substance exposure and neonatal abstinence syndrome. $(\mathrm{n}=279)$

\begin{tabular}{lllll}
\hline Exposure & NAS & & NAS & \\
& $\begin{array}{l}\text { Crude } \\
\text { OR }\end{array}$ & $95 \%$ CI & aOR & $95 \%$ CI \\
& 2.81 & $(1.67,4.73)$ & 3.72 & $(1.54,8.98)$ \\
Opioids only & 0.49 & $(0.24,0.99)$ & 0.83 & $(0.27,2.62)$ \\
Cannabis only & 2.37 & $(1.32,4.26)$ & 3.52 & $(1.13,10.98)$ \\
Poly-substance with opioids & 1.17 & $(0.49,2.83)$ & 1.44 & $(0.32,6.46)$ \\
Other & -- & -- & -- & -- \\
Non-positive (referent) & &
\end{tabular}

NAS: Neonatal abstinence syndrome, OR: Odds ratio, CI: Confidence Interval, aOR:

Adjusted odds ratio, BMI: Body mass index, g: Grams

Adjusted for family visited during hospital admission, reported familial support, history of chronic pain, history of mental health/substance use treatment, smoking packs per day, number of prenatal care visits, maternal pre-pregnancy BMI, and opioid maintenance therapy. 


\section{Chapter 4 Tables}

Table 4.1. Meconium sample required and drug cutoff concentrations

\begin{tabular}{|c|c|c|c|}
\hline Drug & $\begin{array}{l}\text { Sample } \\
\text { amount* }\end{array}$ & Screen cutoff & Confirmation cutoff \\
\hline Cocaine & 0.5 gram & $30 \mathrm{ng} / \mathrm{g}$ & $20 \mathrm{ng} / \mathrm{g}$ \\
\hline \multicolumn{4}{|l|}{ Opioid } \\
\hline Opiates & 0.125 gram & $30 \mathrm{ng} / \mathrm{g}$ & $20 \mathrm{ng} / \mathrm{g}$ \\
\hline Methadone & 0.125 gram & $40 \mathrm{ng} / \mathrm{g}$ & $20 \mathrm{ng} / \mathrm{g}$ \\
\hline Propoxyphene & 1 gram & $75 \mathrm{ng} / \mathrm{g}$ & $10 \mathrm{ng} / \mathrm{g}$ \\
\hline
\end{tabular}

*Minimum volume

ng/g: nanogram /gram 
Table 4.2. Maternal and Neonatal Characteristics by meconium results $(n=381)$

\begin{tabular}{|c|c|c|c|}
\hline Characteristics & Overall & $\begin{array}{l}\text { Meconium } \\
\text { negative for } \\
\text { cocaine or } \\
\text { opioids }\end{array}$ & $\begin{array}{l}\text { Meconium } \\
\text { positive for } \\
\text { cocaine or } \\
\text { opioids }\end{array}$ \\
\hline Total & 381 & $216(56.7 \%)$ & $165(43.3 \%)$ \\
\hline Maternal age, mean (SD) & $26.2(5.1)$ & $26.2(5.1)$ & $26.2(5.2)$ \\
\hline $\begin{array}{l}\text { Years of maternal education, } \\
\text { mean }(\mathrm{SD})\end{array}$ & $12.1(1.9)$ & $12.2(1.8)$ & $11.9(2.1)$ \\
\hline \multicolumn{4}{|l|}{ Marital status } \\
\hline Married & $86(23.6 \%)$ & $54(26.1 \%)$ & $32(20.4 \%)$ \\
\hline Single & $233(64.0 \%)$ & $131(63.3 \%)$ & $102(65.0 \%)$ \\
\hline Other* & $45(12.4 \%)$ & $22(10.6 \%)$ & $23(14.7 \%)$ \\
\hline \multicolumn{4}{|l|}{ Medicaid insurance } \\
\hline Yes & $288(75.6 \%)$ & $158(73.2 \%)$ & $130(78.8 \%)$ \\
\hline No & $93(24.4 \%)$ & $58(26.9 \%)$ & $35(21.2 \%)$ \\
\hline \multicolumn{4}{|l|}{ Residence } \\
\hline Rural & $14(3.9 \%)$ & $9(4.3 \%)$ & $5(3.3 \%)$ \\
\hline Urban & $144(39.7 \%)$ & $83(39.5 \%)$ & $61(39.9 \%)$ \\
\hline Metropolitan & $205(56.5 \%)$ & $118(56.2 \%)$ & $87(56.9 \%)$ \\
\hline $\begin{array}{l}\text { History of drug/mental health } \\
\text { treatment }\end{array}$ & $204(55.9 \%)$ & $107(51.7 \%)$ & $97(61.4 \%)$ \\
\hline SSRI use during pregnancy & $47(12.8 \%)$ & $28(13.3 \%)$ & $19(12.0 \%)$ \\
\hline \multicolumn{4}{|l|}{ Smoking status } \\
\hline Current & $279(75.4 \%)$ & $150(71.4 \%)$ & $129(80.6 \%)$ \\
\hline Former & $53(14.3 \%)$ & $37(17.6 \%)$ & $16(10.0 \%)$ \\
\hline Never & $15(9.4 \%)$ & $23(11.0 \%)$ & $15(9.4 \%)$ \\
\hline Nulliparous & $135(36.5 \%)$ & $89(42.4 \%)$ & $46(28.8 \%)$ \\
\hline Gravidity, median (IQR) & $2.8(1,4)$ & $2(1,4)$ & $2(2,4)$ \\
\hline $\begin{array}{l}\text { Spontaneous abortions, median } \\
\text { (IQR) }\end{array}$ & $0(0,1)$ & $0(0,1)$ & $0(0,1)$ \\
\hline Prenatal visits, mean (SD) & $9.0(6.0)$ & $9.7(6.2)$ & $7.9(5.2)$ \\
\hline \multicolumn{4}{|l|}{ Mode of delivery } \\
\hline Cesarean & $140(36.8 \%)$ & $80(37.0 \%)$ & $60(36.4 \%)$ \\
\hline Vaginal & $241(63.3 \%)$ & $136(63.0 \%)$ & $105(63.6 \%)$ \\
\hline Gestational age, mean (SD) & $37.6(2.4)$ & $37.9(2.3)$ & $37.3(2.5)$ \\
\hline Head width $(\mathrm{cm})$, mean (SD) & $32.8(2.4)$ & $33.0(2.2)$ & $32.7(2.8)$ \\
\hline Length $(\mathrm{cm})$, mean $(\mathrm{SD})$ & $47.6(3.9)$ & $48.1(3.6)$ & $46.9(4.1)$ \\
\hline Weight $(\mathrm{g})$, mean (SD) & $2812.3(620.6)$ & $2885.6(606.6)$ & $2716.0(627.4)$ \\
\hline
\end{tabular}

SD: Standard deviation, SSRI: Selective serotonin reuptake inhibitor, IQR: Interquartile range, cm: centimeters, g: grams

*'Other' marital status incudes women who are divorced, legally separated, or widowed. Percentages do not include missing values. 
Table 4.3. Estimates of in utero drug exposure in 381 mother-infant dyads by neonatal meconium, maternal UDS, and diagnostic codes.

\begin{tabular}{lllll}
\hline & Meconium & $\begin{array}{l}\text { Maternal urine } \\
\text { drug screen* }\end{array}$ & $\begin{array}{l}\text { Maternal } \\
\text { ICD-9-CM }\end{array}$ & $\begin{array}{l}\text { Neonatal } \\
\text { ICD-9-CM }\end{array}$ \\
\hline Opioid positive & $156(40.9 \%)$ & $44(34.4 \%)$ & $141(37 \%)$ & $90(23.6 \%)$ \\
Cocaine positive & $19(5 \%)$ & $7(2.1 \%)$ & $12(3.1 \%)$ & $9(2.4 \%)$ \\
\hline ICD-9-CM: International Classification of Disease, Ninth Revision, Clinical \\
Modification \\
*Values for cocaine-specific maternal urine drug screen were out of 327 \\
mother-infant dyads, because 54 mothers did not have recorded urine drug \\
screen results in their electronic medical records in their labor and delivery \\
hospital admission. Opioid-specific maternal urine drug screens were out of \\
128, because 54 did not have a screen at labor and delivery, and 199 had the \\
new drug panel and thus were not used.
\end{tabular}


Table 4.4. Detection of in utero opioid and cocaine exposure among maternal urine drug screen, substance use diagnosis, and neonatal exposure diagnosis in comparison to meconium drug test.

\begin{tabular}{rccc}
\hline & Maternal UDS & Maternal $I C D-9-C M$ & Neonatal $I C D-9-C M$ \\
\hline Opioid & & & \\
Sensitivity $(95 \%$ CI $)$ & $0.57(0.45,0.69)$ & $0.50(0.42,0.58)$ & $0.27(0.20,0.34)$ \\
Specificity (95\% CI) & $0.90(0.83,0.98)$ & $0.72(0.66,0.78)$ & $0.79(0.73,0.84)$ \\
PPV (95\% CI) & $0.86(0.76,0.97)$ & $0.55(0.47,0.64)$ & $0.47(0.36,0.57)$ \\
NPV (95\% CI) & $0.65(0.55,0.76)$ & $0.68(0.62,0.73)$ & $0.61(0.55,0.66)$ \\
Accuracy (95\% CI) & $0.65(0.58,0.72)$ & $0.63(0.58,0.68)$ & $0.58(0.53,0.62)$ \\
Cocaine & & & \\
Sensitivity (95\% CI) & $0.333(0.12,0.55)$ & $0.368(0.15,0.59)$ & $0.27(0.06,0.46)$ \\
Specificity (95\% CI) & $0.997(0.98,1.00)$ & $0.986(0.97,1.00)$ & $0.99(0.97,1.00)$ \\
PPV (95\% CI) & $0.857(0.42,1.00)$ & $0.583(0.30,0.86)$ & $0.56(0.23,0.88)$ \\
NPV (95\% CI) & $0.963(0.94,0.98)$ & $0.968(0.95,0.99)$ & $0.96(0.94,0.98)$ \\
Accuracy (95\% CI) & $0.963(0.94,0.98)$ & $0.955(0.93,0.98)$ & $0.95(0.93,0.97)$ \\
\hline UDS: Urine drug SCe,
\end{tabular}

UDS: Urine drug scree, ICD-9-CM: International Classification of Disease, Ninth Revision, Clinical Modification, PPV: Positive predictive value, NPV: Negative predictive value, CI:

Confidence interval 


\section{Figures}

\section{Chapter 2 Figures}

Figure 2.1. SAMSHA West Virginia Region and Opioid Treatment Program Center Map

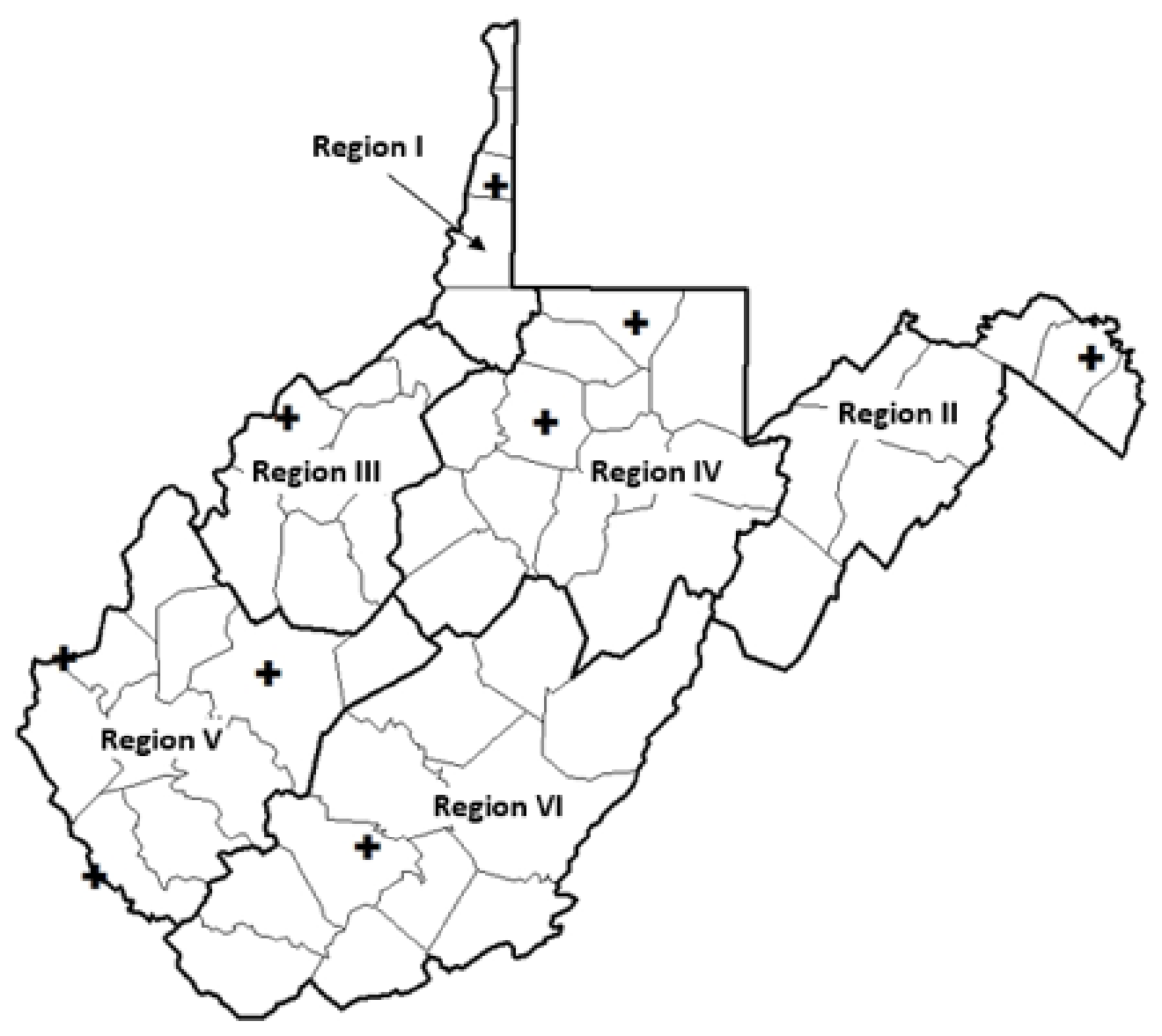

\section{Identification of counties within region}

Region 1: Hancock, Brooke, Ohio, Marshall, Wetzel

Region 2: Pendleton, Grant, Hardy, Mineral, Hampshire, Morgan, Berkeley, Jefferson

Region 3: Tyler, Pleasants, Ritchie, Wood, Wirt, Jackson, Roane, Calhoun

Region 4: Monongalia, Preston, Tucker, Randolph, Barbour, Upshur, Braxton, Gilmer, Lewis, Doddridge, Harrison, Taylor, Marion

Region 5: Pocahontas, Webster, Greenbrier, Monroe, Summers, Mercer, McDowell, Wyoming, Raleigh, Fayette, Nicholas

Region 6: Clay, Kanawha, Putnam, Mason, Cabell, Lincoln, Wayne, Boone, Logan, Mingo 
Figure 2.2. West Virginia Regional and Hospital Neonatal Abstinence Syndrome Incidence Rate per 1,000 Live Births

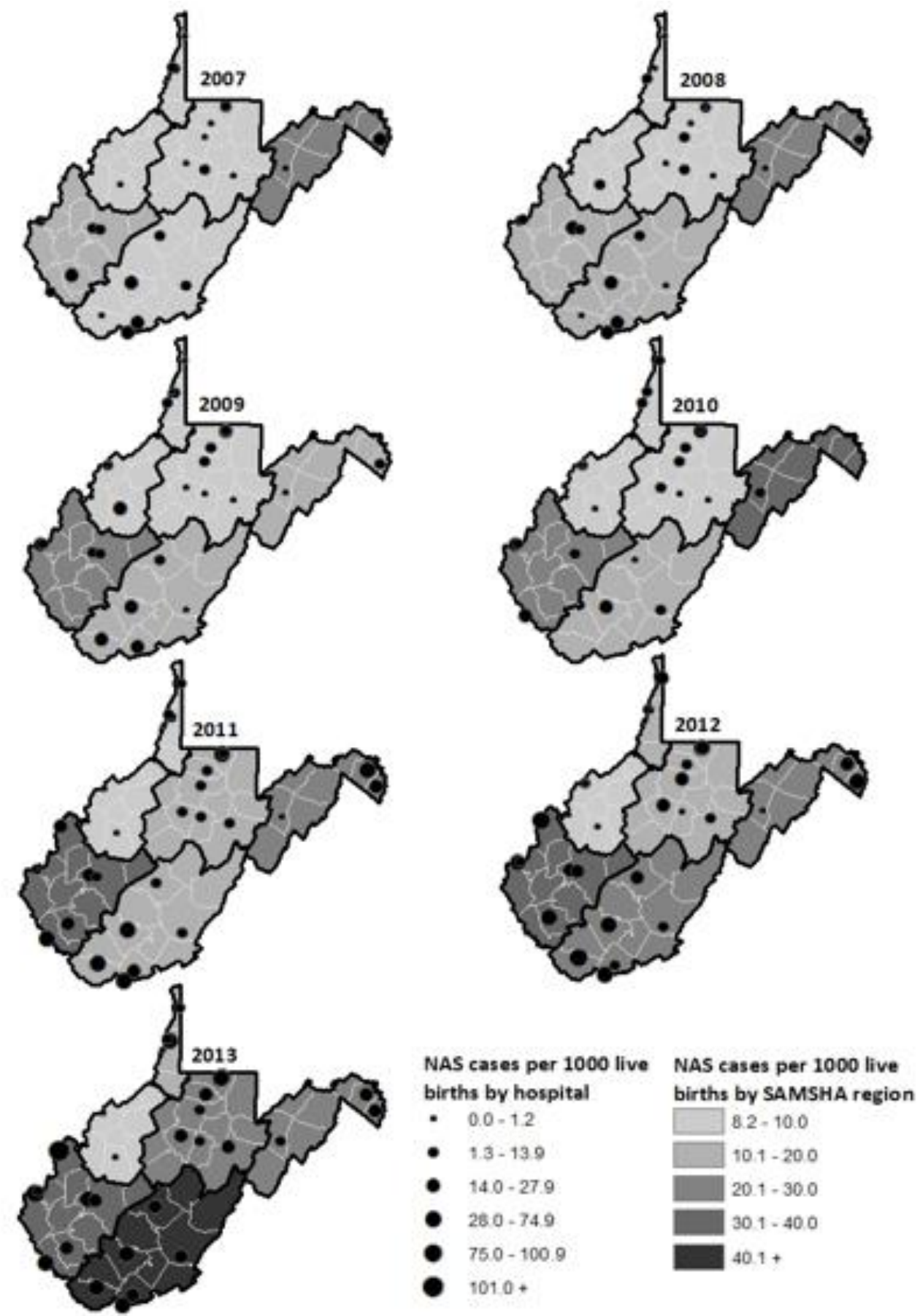


Figure 2.3: Number of inpatient hospitalizations resulting from exposure to noxious substances, West Virginia, 2007-2013

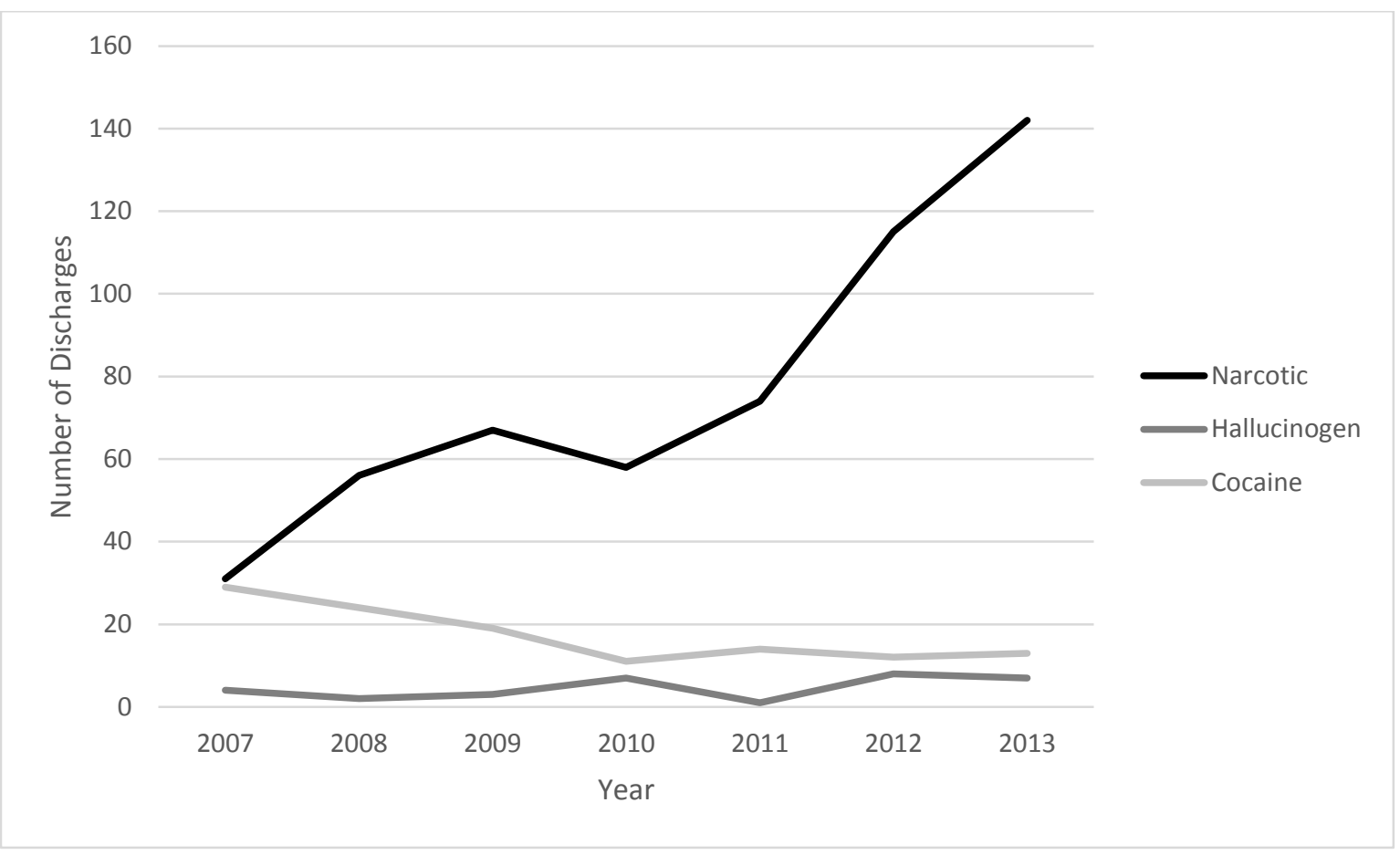




\section{Chapter 3 Figure}

Figure 3.1. Biopsychosocial approach to understanding neonatal abstinence syndrome

- History of substance use/mental health treatment

- History of chronic pain

- Opioid maintenance therapy enrollment

\section{Biological}

-Maternal age

- Nulliparous

- Gravidity

- Delivery type

-Pre-pregnancy BMI

- Neonatal sex

- Gestational age

- Neonatal head, length, \& weight

measurements

- Number of prenatal care visits

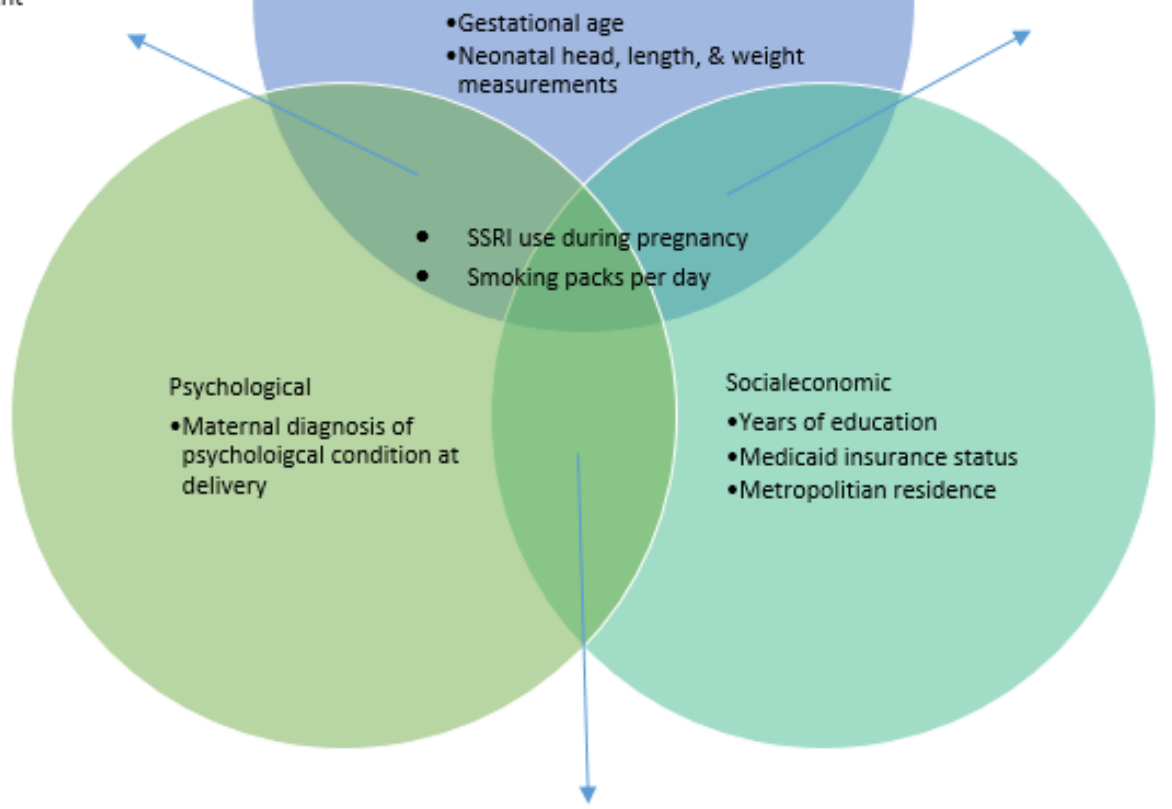

- Marital Status

- Family visited at hospital admission

- Reported familial support

- Reported or suspected physical abuse 


\section{Appendixes}

A.

NEONATAL ABSTINENCE SCORING SYSTEM

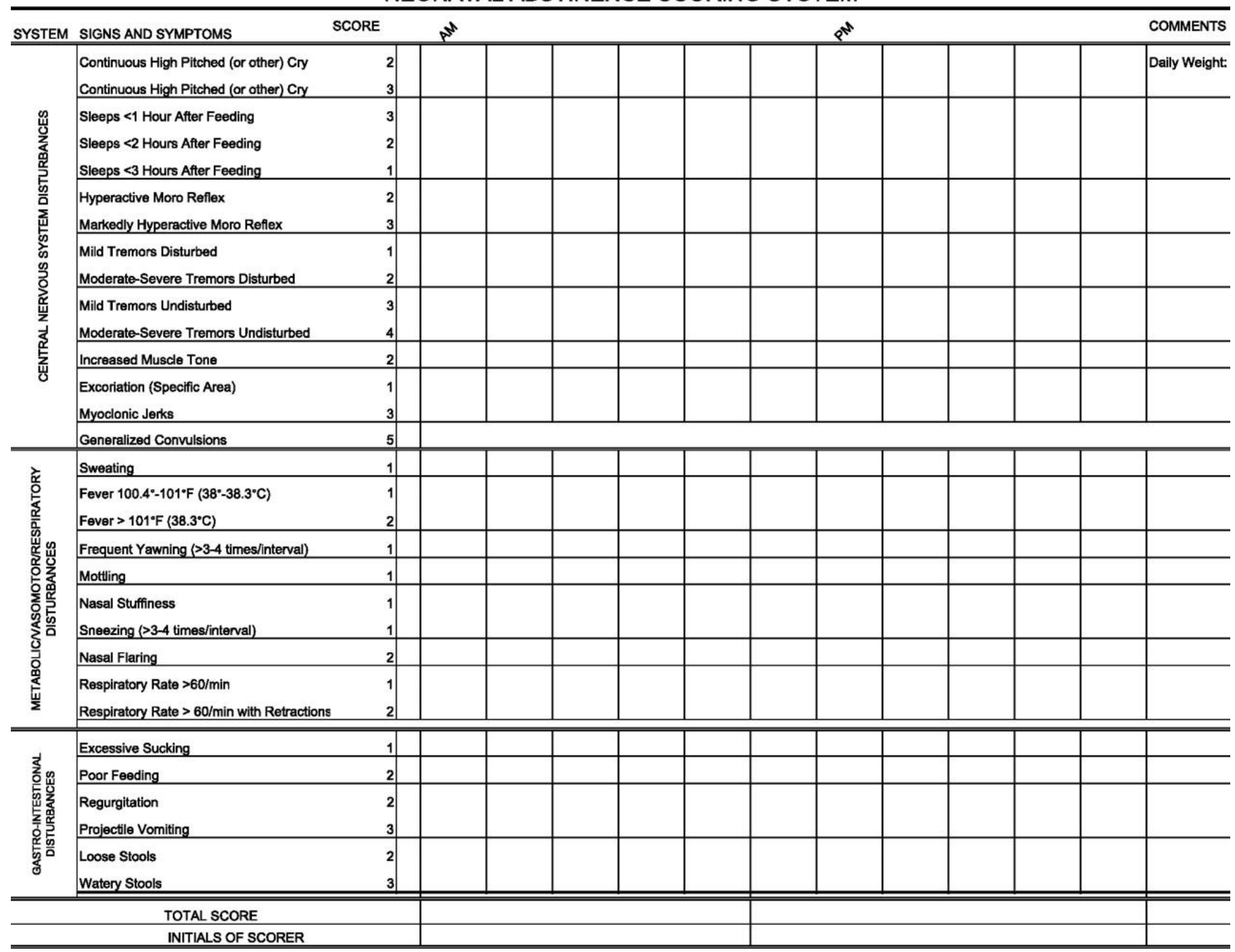


B.

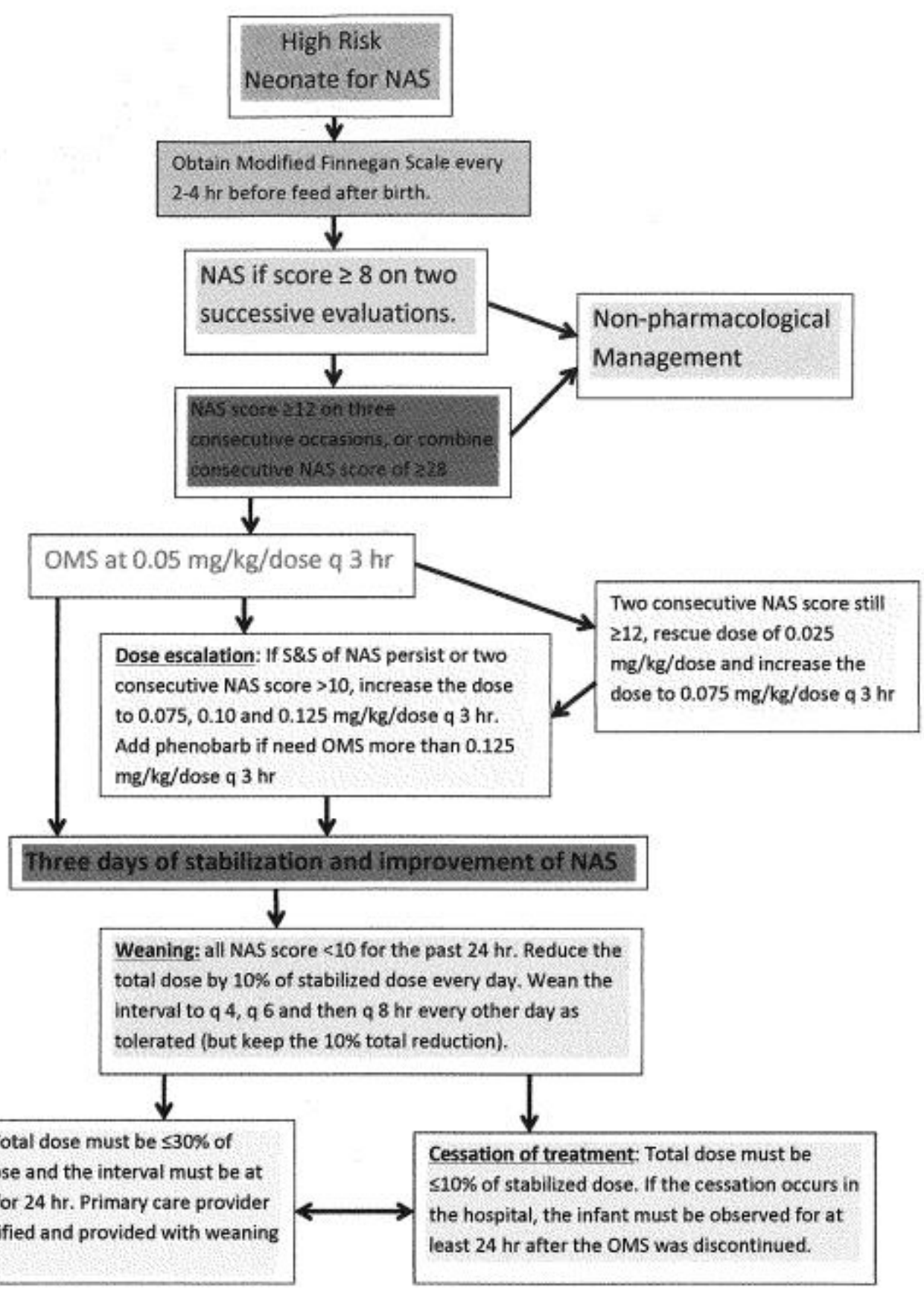

Dose adjustment: Switching from OMS to morphine injection must be discussed with PedsPharmD as necessary 
Stabilization Dose: $0.05 \mathrm{mg} / \mathrm{kg}$ every 3 hours

Wean Step 1: $0.045 \mathrm{mg} / \mathrm{kg}$ every 3 hours

Wean Step 2: $0.053 \mathrm{mg} / \mathrm{kg}$ every 4 hours

Wean Step 3: $0.047 \mathrm{mg} / \mathrm{kg}$ every 4 hours

Wean Step 4: $0.06 \mathrm{mg} / \mathrm{kg}$ every 6 hours

Wean Step 5: $0.05 \mathrm{mg} / \mathrm{kg}$ every 6 hours

Wean Step 6: $0.053 \mathrm{mg} / \mathrm{kg}$ every 8 hours

Wean Step 7: $0.04 \mathrm{mg} / \mathrm{kg}$ every 8 hours (home going dose)

Wean Step $8: 0.04 \mathrm{mg} / \mathrm{kg}$ every 12 hours

Wean Step 9: $0.04 \mathrm{mg} / \mathrm{kg}$ every 24 hours

Stabilization Dose: $0.075 \mathrm{mg} / \mathrm{kg}$ every 3 hours

Wean Step 1: $0.067 \mathrm{mg} / \mathrm{kg}$ every 3 hours

Wean Step 2: $0.08 \mathrm{mg} / \mathrm{kg}$ every 4 hours

Wean Step 3: $0.07 \mathrm{mg} / \mathrm{kg}$ every 4 hours

Wean Step 4: $0.09 \mathrm{mg} / \mathrm{kg}$ every 6 hours

Wean Step 5: $0.075 \mathrm{mg} / \mathrm{kg}$ every 6 hours

Wean Step 6: $0.08 \mathrm{mg} / \mathrm{kg}$ every 8 hours

Wean Step 7: $0.06 \mathrm{mg} / \mathrm{kg}$ every 8 hours (home going dose)

Wean Step $8: 0.06 \mathrm{mg} / \mathrm{kg}$ every 12 hours

Wean Step 9: $0.06 \mathrm{mg} / \mathrm{kg}$ every 24 hours

Stabilization Dose: $0.1 \mathrm{mg} / \mathrm{kg}$ every 3 hours

Wean Step 1: $0.09 \mathrm{mg} / \mathrm{kg}$ every 3 hours

Wean Step 2: $0.1 \mathrm{mg} / \mathrm{kg}$ every 4 hours

Wean Step 3: $0.093 \mathrm{mg} / \mathrm{kg}$ every 4 hours

Wean Step 4: $0.12 \mathrm{mg} / \mathrm{kg}$ every 6 hours

Wean Step 5: $0.1 \mathrm{mg} / \mathrm{kg}$ every 6 hours

Wean Step 6: $0.1 \mathrm{mg} / \mathrm{kg}$ every 8 hours

Wean Step 7: $0.08 \mathrm{mg} / \mathrm{kg}$ every 8 hours (home going dose)

Wean Step $8: 0.08 \mathrm{mg} / \mathrm{kg}$ every 12 hours

Wean Step 9: $0.08 \mathrm{mg} / \mathrm{kg}$ every 24 hours

Stabilization Dose: $0.125 \mathrm{mg} / \mathrm{kg}$ every 3 hours

Wean Step 1: $0.112 \mathrm{mg} / \mathrm{kg}$ every 3 hours

Wean Step 2: $0.133 \mathrm{mg} / \mathrm{kg}$ every 4 hours

Wean Step 3: $0.116 \mathrm{mg} / \mathrm{kg}$ every 4 hours

Wean Step $4: 0.15 \mathrm{mg} / \mathrm{kg}$ every 6 hours

Wean Step 5: $0.125 \mathrm{mg} / \mathrm{kg}$ every 6 hours

Wean Step 6: $0.133 \mathrm{mg} / \mathrm{kg}$ every 8 hours

Wean Step 7: $0.1 \mathrm{mg} / \mathrm{kg}$ every 8 hours (home going dose)

Wean Step 8: $0.1 \mathrm{mg} / \mathrm{kg}$ every 12 hours

Wean Step 9: $0.1 \mathrm{mg} / \mathrm{kg}$ every 24 hours 
C.

\section{Confidential}

\section{Manual Data Extraction}

Record ID

MRN (9 spaces)

Employment status

Years of education

Family visited at hospital?

History of drug abuse

Smoking status

Smoking Packs per day

Number of YEARS smoked

History of treatment for alcohol/drug/or behavioral issues

Any current treatment for alcohol/drug/or behavioral issues

Current caffeine use

Current gravidity

Number of previous abortions

Is the mother nulliparous?

Opioid treatment program during pregnancy?

If yes, what type of ORT?

Was pertosin administered during L/D?

$09 / 27 / 2015: 4: 04 \mathrm{pm}$

\begin{tabular}{l}
\hline \\
\hline currently employed \\
Oot employed \\
unknown
\end{tabular}

Family at hospital

O Family not at hospital

OUnknown

Yes
Ono
Onknown

O Current

OPrevious

O Never

(A 1 Decimal place)

(स 1 Decimal place)

OYes

O No

O Unknown

OYes

ONo

OUnknown

OYes

No

O Unknown

YYes

O No

O Unknown

(*Not counting current birth *other children at home??)

OYes

No

OUnknown

((BMT or MMT))

O Buprenorphine (BMT)

Methadone (MMT)

OUnkown

Y Yes

No

O Unknown 


\section{Confidential}

Was anesthesia given at delivery?

If yes, what type of anesthesia?

Was there usual coping mechanisms reported?

History of chronic pain?

Did the mother take SSRIs during pregnancy?

Were there reported signs of physical abuse?

Mother's height (meters)

Mother's weight (kgs) before pregnancy

Mother's weight (kgs) at delivery?

Weight gain during pregnancy in kgs

Mother's BMI at delivery_INCORRECT

Mother's BMI at delivery_CORRECT

Reported family social support.

Date and time of delivery

Pain score 1

Time of pain score 1

Pain score 2

Time of pain score 2

09/27/2015 4:04pm
OYes

No

Unknown

OLocal

Spinal (regional)

O Epidural (regional)

O General

Other

OUnknown

OYes

No

Unknown

(coder needs to be intuitive )

OYes

No

OUnknown

OYes

O No

OUnknown

(Include: fluoxetine, paroxetine hydrochloride, citalopram hydrobromide, sertraline hydrochloride, and the serotonin-noradrenaline reuptake inhibitor venlafaxine hydrochloride, recorded anytime during pregnancy or just in the 3rd trimester. )

OYes

No

O Unknown

(METERS)

(स्क्न in KILOGRAMS)

(स्कin KILOGRAMS)

(स्will be presented in kgs)

(A्AGNORE)

(क्न्नORRECT BMFक्नKG, METER)

OYes

No

OUnknown

(Month, day, year then hour and minute)

(सALL POSTPARTUM)

(AALLL POSTPARTUM) 


\section{Confidential}

Pain score 3

Time of pain score 3

Pain score 4

Time of pain score 4

Pain score 5

Time of pain score 5

Pain score 6

Time of pain score 6

Pain score 7

Time of pain score 7

Pain score 8

Time of pain score 8

Pain score 9

Time of pain score 9

Pain score 10

Time of pain score 10

Pain score 11

Time of pain score 11

Pain score 12

Time of pain score 12

Pain score 13

Time of pain score 13

Pain score 14

Time of pain score 14

Pain score 15

Time of pain score 15

Pain score 16

Time of pain score 16

Pain score 17

Time of pain score 17

Pain score 18

Time of pain score 18

Pain score 19

Time of pain score 19

09/27/2015 4:04pm

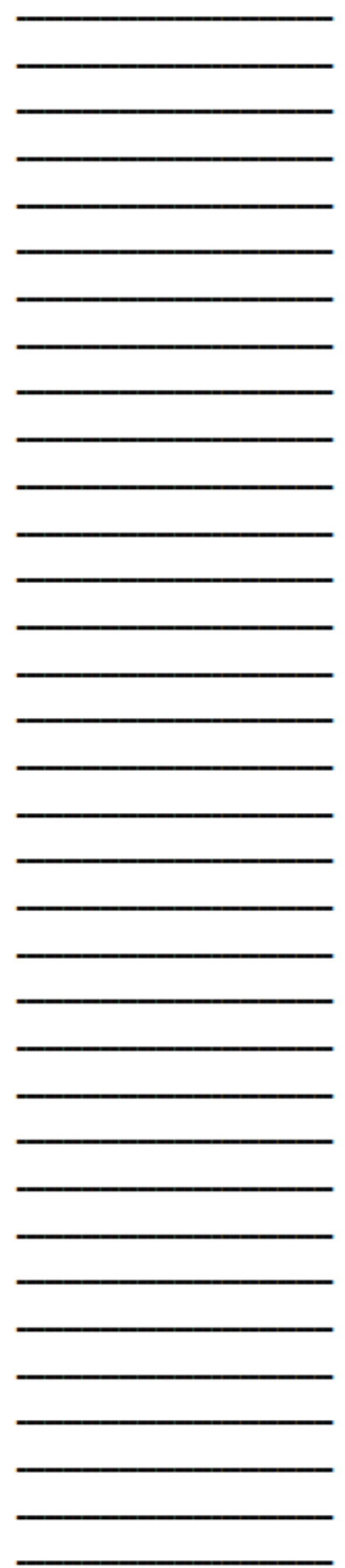

www.projectredcap.org 


\section{Confidential}

Pain score 20

Time of pain score 20

Pain score 21

Time of pain score 21

Pain score 22

Time of pain score 22

Pain score 23

Time of pain score 23

Pain score 24

Time of pain score 24

Pain score 25

Time of pain score 25

Did the baby have NAS?

If the baby had NAS, did he/she receive pharmacological treatment?

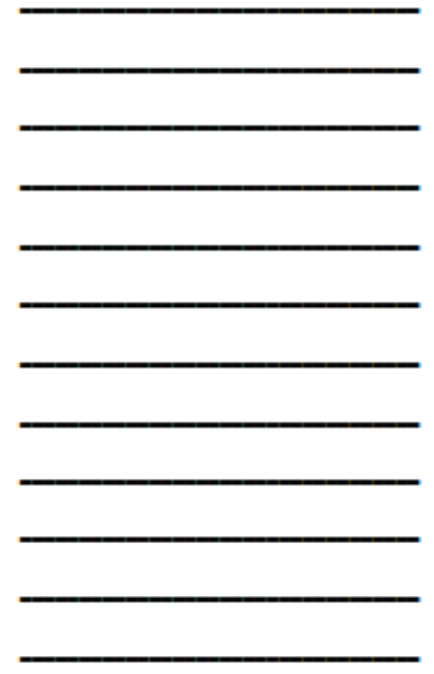

OYes

No

(Look at data set)

Y Yes

No

Maybe

(NAS diagnosis is already in data set)

If the baby had NAS, what was his/her peak Finnegan score?

If the baby had NAS, what day did he/she score their peak Finnegan score?

Gestation age (wks)

Neonatal head circumference $(\mathrm{cm})$

Neonatal birth length $(\mathrm{cm})$

(report in $\mathrm{cm}$ )

(in CENTIMETERS)

Neonatal birth weight (GRAMS)

(in GRAMS) 
Confidential

Page 5 of 5

Neonatal diagnosis primary or secondary

hypogicaemia

Yes

Jaundice

$\mathrm{O}$

$\mathrm{O}$

Seizures

$\mathrm{O}$

microcephaly

○

thrombocytosis

O

arrhythmias

○

prolonged electrocardiographic

$\mathrm{O}$

QTc interval

under developed eyes/sight

O

decreased fetal oxygen supply

09/27/2015 4:04pm

\section{www.projectredcap.org REDCap}

Note: This is the data that was manually collected (i.e., not provided in full by the hospital decision support analyst.) 
D.

Confidential

\section{Dissertation Exposure}

DISS exposure Excluded MEC

Page 1 of 1

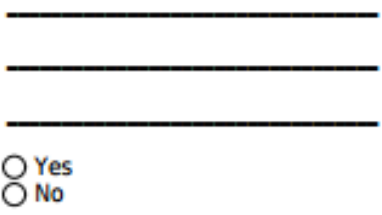

ICD-9 760.7X

7600

760.72

760.73

760.75

No ICD-9 760.7X Listed

Was there a meconium, CTS, or both taken on the baby?

NO Drug Screen Listed

\begin{tabular}{|c|c|c|}
\hline Meconium & & \\
\hline & Yes & No \\
\hline CANNABINOIDS & 0 & $\mathrm{O}$ \\
\hline COCAINE & $\mathrm{O}$ & $\mathrm{O}$ \\
\hline OPIATES & 0 & $\mathrm{O}$ \\
\hline PHENCYCUIDINE & 0 & $\mathrm{O}$ \\
\hline AMPHETAMINES & 0 & $\mathrm{O}$ \\
\hline BARBITUATES & $\mathrm{O}$ & $\mathrm{O}$ \\
\hline METHADONE AND METABOLITE & $\mathrm{O}$ & $\mathrm{O}$ \\
\hline BENZODIAZEPINES & $\mathrm{O}$ & $\mathrm{O}$ \\
\hline PROPOXYPHENE & 0 & 0 \\
\hline
\end{tabular}

09/27/2015 4:08pm

Note: I did not have to collect umbilical cord tissue data because it was provided by the hospital decision support analyst. 
E.

Meconium Drug Cutoff Concentrations, ARUP Laboratory*

\begin{tabular}{lll} 
Drug & Screen cutoff & $\begin{array}{l}\text { Confirmation } \\
\text { cutoff }\end{array}$ \\
\hline Marijuana & $30 \mathrm{ng} / \mathrm{g}$ & $5 \mathrm{ng} / \mathrm{g}$ \\
Cocaine & $30 \mathrm{ng} / \mathrm{g}$ & $20 \mathrm{ng} / \mathrm{g}$ \\
Opiates & $30 \mathrm{ng} / \mathrm{g}$ & $2 \mathrm{ng} / \mathrm{g}$ \\
Phencyclidine & $15 \mathrm{ng} / \mathrm{g}$ & $10 \mathrm{ng} / \mathrm{g}$ \\
Amphetamines & $30 \mathrm{ng} / \mathrm{g}$ & $20 \mathrm{ng} / \mathrm{g}$ \\
Barbiturates & $75 \mathrm{ng} / \mathrm{g}$ & $50 \mathrm{ng} / \mathrm{g}$ \\
Methadone & $40 \mathrm{ng} / \mathrm{g}$ & $10 \mathrm{ng} / \mathrm{g}$ \\
Benzodiazepines & $75 \mathrm{ng} / \mathrm{g}$ & $20 \mathrm{ng} / \mathrm{g}$ \\
Propoxyphene & $75 \mathrm{ng} / \mathrm{g}$ & $10 \mathrm{ng} / \mathrm{g}$ \\
\hline
\end{tabular}

ARUP: Associated Regional and University Pathologists laboratories in Salt Lake City, Utah, ng/g: nanogram /gram

*The methodology utilized is quantitative liquid chromatography-tandem mass spectrometry 
Umbilical Cord Tissue Drug Cutoff Concentrations, ARUP Laboratory*

\begin{tabular}{|c|c|c|}
\hline Drug class & Drug & Screen cutoff \\
\hline \multicolumn{3}{|l|}{ Opioids } \\
\hline & Buprenorphine & $2 \mathrm{ng} / \mathrm{g}$ \\
\hline & Buprenorphine-G & $8 \mathrm{ng} / \mathrm{g}$ \\
\hline & Codeine & $6 \mathrm{ng} / \mathrm{g}$ \\
\hline & Dihydrocodeine & $4 \mathrm{ng} / \mathrm{g}$ \\
\hline & Fentanyl & $1 \mathrm{ng} / \mathrm{g}$ \\
\hline & Hydrocodone & $6 \mathrm{ng} / \mathrm{g}$ \\
\hline & Hydromorphone & $4 \mathrm{ng} / \mathrm{g}$ \\
\hline & Meperidine & $2 \mathrm{ng} / \mathrm{g}$ \\
\hline & Methadone & $10 \mathrm{ng} / \mathrm{g}$ \\
\hline & EDDP & $10 \mathrm{ng} / \mathrm{g}$ \\
\hline & 6-Acetylmorphine & $4 \mathrm{ng} / \mathrm{g}$ \\
\hline & Morphine & $4 \mathrm{ng} / \mathrm{g}$ \\
\hline & Naloxone & $8 \mathrm{ng} / \mathrm{g}$ \\
\hline & Naltrexone & $8 \mathrm{ng} / \mathrm{g}$ \\
\hline & Oxycodone & $4 \mathrm{ng} / \mathrm{g}$ \\
\hline & Oxymorphone & $4 \mathrm{ng} / \mathrm{g}$ \\
\hline & Propoxyphene & $10 \mathrm{ng} / \mathrm{g}$ \\
\hline & Tapentadol & $2 \mathrm{ng} / \mathrm{g}$ \\
\hline & Tramadol & $2 \mathrm{ng} / \mathrm{g}$ \\
\hline & N-Desmethyltramadol & $2 \mathrm{ng} / \mathrm{g}$ \\
\hline & O-Desmethyltramadol & $2 \mathrm{ng} / \mathrm{g}$ \\
\hline \multicolumn{3}{|l|}{ Stimulants } \\
\hline & Amphetamine & $8 \mathrm{ng} / \mathrm{g}$ \\
\hline & Benzoylecgonine & $8 \mathrm{ng} / \mathrm{g}$ \\
\hline & M-Oh-Benzoylecgonine & $8 \mathrm{ng} / \mathrm{g}$ \\
\hline & Cocaethylene & $8 \mathrm{ng} / \mathrm{g}$ \\
\hline & Cocaine & $8 \mathrm{ng} / \mathrm{g}$ \\
\hline & MDMA- Ecstasy & $8 \mathrm{ng} / \mathrm{g}$ \\
\hline & MDA & $8 \mathrm{ng} / \mathrm{g}$ \\
\hline & MDEA- EVE & $8 \mathrm{ng} / \mathrm{g}$ \\
\hline & Methamphetamine & $8 \mathrm{ng} / \mathrm{g}$ \\
\hline & Phentermine & $8 \mathrm{ng} / \mathrm{g}$ \\
\hline \multicolumn{3}{|c|}{ Sedatives-Hypnotics } \\
\hline & Alprazolam & $5 \mathrm{ng} / \mathrm{g}$ \\
\hline & Alpha-OH-Alprazolam & $5 \mathrm{ng} / \mathrm{g}$ \\
\hline & Butalbital & $40 \mathrm{ng} / \mathrm{g}$ \\
\hline & Clonazepam & $5 \mathrm{ng} / \mathrm{g}$ \\
\hline & 7-Aminoclonazepam & $5 \mathrm{ng} / \mathrm{g}$ \\
\hline & Diazepam & $5 \mathrm{ng} / \mathrm{g}$ \\
\hline & Flunitrazepam & $5 \mathrm{ng} / \mathrm{g}$ \\
\hline & 7-Aminoflunitrazepam & $5 \mathrm{ng} / \mathrm{g}$ \\
\hline & Flurazepam & $5 \mathrm{ng} / \mathrm{g}$ \\
\hline
\end{tabular}


Phencyclidine

$\begin{array}{ll}\text { Desalkylflurazepam } & 10 \mathrm{ng} / \mathrm{g} \\ \text { 2-OH-Ethylflurazepam } & 10 \mathrm{ng} / \mathrm{g} \\ \text { Lorazepam } & 5 \mathrm{ng} / \mathrm{g} \\ \text { Midazolam } & 5 \mathrm{ng} / \mathrm{g} \\ \text { Alpha-OH-Midazolam } & 5 \mathrm{ng} / \mathrm{g} \\ \text { Nitrazepam } & 5 \mathrm{ng} / \mathrm{g} \\ \text { Nordiazepam } & 5 \mathrm{ng} / \mathrm{g} \\ \text { Oxazepam } & 5 \mathrm{ng} / \mathrm{g} \\ \text { Phenobarbital } & 20 \mathrm{ng} / \mathrm{g} \\ \text { Secobarbital } & 40 \mathrm{ng} / \mathrm{g} \\ \text { Temazepam } & 5 \mathrm{ng} / \mathrm{g} \\ \text { Triazolam } & 5 \mathrm{ng} / \mathrm{g} \\ \text { Alpha-OH-Triazolam } & 5 \mathrm{ng} / \mathrm{g} \\ \text { Zolpidem } & 10 \mathrm{ng} / \mathrm{g}\end{array}$

Marijuana

Phencyclidine-PCP $4 \mathrm{ng} / \mathrm{g}$

Marijuana metabolite $\quad 150 \mathrm{pg} / \mathrm{g}$

ARUP: Associated Regional and University Pathologists laboratories in Salt Lake City, Utah, ng/g: nanogram /gram, pg/g: picogram/gram

*The methodology utilized is qualitative liquid chromatography-time of flight mass spectrometry and enzyme-linked immunosorbent assay 
F.

Definitions:

Sensitivity $=\mathrm{TP} /(\mathrm{TP}+\mathrm{FN})=($ Number of true positive assessment $) /($ Number of all positive assessment $)$ Specificity $=\mathrm{TN} /(\mathrm{TN}+\mathrm{FP})=($ Number of true negative assessment $) /($ Number of all negative assessment $)$ Accuracy $=(\mathrm{TN}+\mathrm{TP}) /(\mathrm{TN}+\mathrm{TP}+\mathrm{FN}+\mathrm{FP})=($ Number of correct assessments)/Number of all assessments)

Positive Predictive Value $(\mathrm{PPV})=\mathrm{TP} /(\mathrm{TP}+\mathrm{FP})=$ Number of true positives/Number of test positives

Negative Predictive Value $(\mathrm{NPV})=\mathrm{TN} /(\mathrm{TN}+\mathrm{FN})=$ Number of true negatives/Number of test negatives

\begin{tabular}{|l|l|l|l|}
\hline Opioid Meconium \& Maternal ICD-9-CM Diagnosed (opioid) \\
\hline Meconium results & Mom diagnosed & Mom not diagnosed & Total \\
\hline Positive & 78 & 78 & 156 \\
\hline Negative & 63 & 162 & 225 \\
\hline
\end{tabular}

Sensitivity: 0.50, ASE confidence limits $(0.419,0.581)$

Specificity: 0.72, ASE confidence limits $(0.6613,0.7787)$

PPV: 0.5532, ASE confidence limits $(0.4711,0.6353)$

NPV: 0.6750, ASE confidence limits $(0.6157,0.7343)$

Accuracy: 0.6299, ASE confidence limits (0.5814, 0.6784)

\begin{tabular}{|l|l|l|l|}
\hline Opioid Meconium \& Maternal UDS (opioid) \\
\hline Meconium results & + UDS & - UDS & Total \\
\hline Positive & 38 & 29 & 67 \\
\hline Negative & 6 & 55 & 61 \\
\hline
\end{tabular}

Sensitivity: 0.5672, ASE confidence limits $(0.4485,0.6858)$

Specificity: 0.9016, ASE confidence limits $(0.8269,0.9764)$

PPV: 0.8636, ASE confidence limits $(0.7622,0.9650)$

NPV: 0.6548 , ASE confidence limits $(0.5531,0.7564)$

Accuracy: 0.6538, ASE confidence limits $(0.5847,0.7227)$

\begin{tabular}{|l|l|l|l|}
\hline Opioid Meconium \& Neonatal exposure diagnosis (opioid) \\
\hline Meconium results & Neonatal diagnosed & Neonatal not diagnosed & Total \\
\hline Positive & 42 & 114 & 156 \\
\hline Negative & 48 & 177 & 225 \\
\hline
\end{tabular}

Sensitivity: 0.2692, ASE confidence limits (0.1996, 0.3388)

Specificity: 0.7867, ASE confidence limits $(0.7331,0.8402)$

PPV: 0.4667, ASE confidence limits $(0.3636,0.5697)$

NPV: 0.6082, ASE confidence limits $(0.5522,0.6643)$

Accuracy: 0.5748, ASE confidence limits (0.5252, 0.6244) 


\begin{tabular}{|l|l|l|l|}
\hline \multicolumn{5}{|l|}{ Cocaine Meconium \& Maternal ICD-9-CM Diagnosed (cocaine) } \\
\hline Meconium results & Mom diagnosed & Mom not diagnosed & Total \\
\hline Positive & 7 & 12 & 19 \\
\hline Negative & 5 & 357 & 362 \\
\hline
\end{tabular}

Sensitivity: 0.3684, ASE confidence limits $(0.1515,0.5853)$

Specificity: 0.9862 , ASE confidence limits $(0.9742,9982)$

PPV: 0.5833, ASE confidence limits $(0.3044,0.8623)$

NPV: 0.9675, ASE confidence limits (0.9494, 0.9856)

Accuracy: 0.9554, ASE confidence limits (0.9346, 0.9761)

\begin{tabular}{|l|l|l|l|}
\hline \multicolumn{4}{|l|}{ Cocaine Meconium \& Maternal UDS (cocaine) } \\
\hline Meconium results & + UDS & - UDS & Total \\
\hline Positive & 38 & 29 & 67 \\
\hline Negative & 6 & 55 & 61 \\
\hline
\end{tabular}

Sensitivity: 0.5672, ASE confidence limits $(0.4485,0.6858)$

Specificity: 0.9016, ASE confidence limits $(0.8269,0.9764)$

PPV: 0.8636, ASE confidence limits $(0.7622,0.9650)$

NPV: 0.6548, ASE confidence limits $(0.5531,0.7564)$

Accuracy: 0.6538 , ASE confidence limits $(0.5847,0.7227)$

\begin{tabular}{|l|l|l|l|}
\hline \multicolumn{5}{|l|}{ Cocaine Meconium \& Neonatal exposure diagnosis (cocaine) } \\
\hline Meconium results & Neonatal diagnosed & Neonatal not diagnosed & Total \\
\hline Positive & 5 & 14 & 19 \\
\hline Negative & 4 & 358 & 362 \\
\hline
\end{tabular}

Sensitivity: 0.2692 , ASE confidence limits $(0.0652,0.4612)$

Specificity: 0.9890, ASE confidence limits $(0.9720,0.9970)$

PPV: 0.5556, ASE confidence limits $(0.2309,0.8802)$

NPV: 0.9624, ASE confidence limits $(0.9430,0.9817)$

Accuracy: 0.9528 , ASE confidence limits $(0.9315,0.9741)$ 